\title{
BATTEN DISEASE BEYOND THE BLIND SPOTS
}





\section{BATTEN DISEASE - BEYOND THE BLIND SPOTS}

Willemijn Kuper 


\section{Colophon}

The studies described in this thesis were financially supported by the Beat Batten Foundation and the Bartiméus Foundation.

Printing of this thesis was financially supported by the Beat Batten Foundation and the Bartiméus Foundation.

Cover idea by Willemijn Kuper

Cover design by Isabel de Waard, persoonlijkproefschrift.nl

Layout and design by Isabel de Waard, persoonlijkproefschrift.nl

Printing: Ridderprint | www.ridderprint.nl

ISBN: 978-94-6375-779-9

(c) W.F.E. Kuper, 2020. All rights reserved.

No part of this thesis may be reproduced, stored in a retrieval system or transmitted in any form or by any means without prior written permission of the author. 


\title{
BATTEN DISEASE - BEYOND THE BLIND SPOTS
}

DE ZIEKTE VAN BATTEN - VOORBIJ DE BLINDE VLEKKEN

(met een samenvatting in het Nederlands)

\author{
Proefschrift \\ ter verkrijging van de graad van doctor aan de \\ Universiteit Utrecht \\ op gezag van de \\ rector magnificus, prof dr. H.R.B.M. Kummeling, \\ ingevolge het besluit van het college voor promoties \\ in het openbaar te verdedigen op
}

woensdag 14 oktober 2020 des middags te 12.45 uur

door

Willemijn Fennie Eelkje Kuper

geboren op 12 december 1991

te Nijmegen 


\section{Promotoren:}

Prof. dr. E.E.S. Nieuwenhuis

Prof. dr. M.M. van Genderen

\section{Copromotor:}

Dr. P.M. van Hasselt 
Aan Roel

Aan mijn ouders 



\section{CONTENTS}

Chapter 1 General introduction and outline of this thesis

\section{Part 1 | Early recognition of CLN3 disease}

Chapter 2 Timing of cognitive decline in CLN3 disease

Chapter 3 Motor function impairment is an early sign of CLN3 disease

Chapter 4 Differentiating ophthalmological characteristics of CLN3 disease (Batten disease)

68

Part 2 | Expanding the phenotypic spectrum of CLN3 disease

\begin{tabular}{ll}
\hline Chapter $\mathbf{5}$ & $\begin{array}{l}\text { The C.1A }>\text { C start codon mutation in CLN3 is associated } \\
\text { with a protracted disease course }\end{array}$ \\
Chapter $\mathbf{6}$ & $\begin{array}{l}\text { A case of unexpected adult-onset neurologic decline in } \\
\text { CLN3-associated retinal degeneration }\end{array}$ \\
\hline
\end{tabular}

Part 3 | Storage material accumulation in CLN3 disease revisited

\begin{tabular}{ll}
\hline Chapter 7 & $\begin{array}{l}\text { Quantifying lymphocyte vacuolization serves as a measure } \\
\text { of CLN3 disease severity }\end{array}$ \\
Chapter 8 & $\begin{array}{l}\text { Storage material accumulation in the neuronal ceroid } \\
\text { lipofuscinoses revisited }\end{array}$
\end{tabular}

Chapter 9 General discussion

Addendum

Chapter 10 Summary and Nederlandse samenvatting

List of abbreviations

List of publications

Dankwoord

Curriculum Vitae (Nederlands) 



\section{CHAPTER GENERAL INTRODUCTION AND OUTLINE OF THIS THESIS}




\section{BATTEN DISEASE - AN ENIGMATIC DISEASE}

In 1903, the British ophthalmologist Frederick Batten reported on two sisters who, after an initially normal development, rapidly lost their vision, and subsequently deteriorated mentally and physically. ${ }^{1}$ Interestingly, Batten was most probably not the first to have reported on this disease, as, in 1826 , Christian Stengel already described four siblings who were born healthy but similarly lost their vision accompanied by the occurrence of seizures and progressive psychomotor decline. ${ }^{2}$ Following Batten's report, several others, whose names are sometimes still used to refer to the disease (Mayou, Vogt, Spielmeyer) reported on patients with a similar disease course. ${ }^{3}$

In modern terminology, all these reports likely described patients suffering from (juvenile) neuronal ceroid lipofuscinosis (NCL). All disorders characterized clinically by onset of blindness and dementia in childhood and histologically by some sort of storage material accumulation were initially grouped together, and referred to as the "amaurotic family idiocies". Later, with increasing insight in the composition of the accumulated storage material, it was recognized that these "amaurotic family idiocies" comprised of at least two (groups of) disorders: Tay-Sachs disease with accumulation of GM2 ganglioside (not discussed in this thesis) and the NCLs. The NCLs were named after the ceroidlipofuscin like appearance of the stored material, predominantly affecting neuronal tissues. ${ }^{4}$

In 1995, CLN3, the gene responsible for (most cases of) juvenile NCL was identified followed by many of the other genes underlying the NCLs in the years thereafter..$^{5-8}$ In these years of exciting genetic discoveries, it was expected that the NCLs and other genetic diseases would soon be curable. However, this promise has yet to be fulfilled. The NCLs have shown to remain enigmatic; both on a clinical and on a pathophysiological level. Compared to the early years of genetic discoveries, we now increasingly understand how little we still understand. ${ }^{9}$

\section{THE NEURONAL CEROID LIPOFUSCINOSES}

The NCLs are unified (i) clinically by degeneration of the brain and in most NCLS also by degeneration of the retina and (ii) pathologically by the ubiquitous accumulation of auto fluorescent ceroid lipofuscin-like storage material. ${ }^{10}$ Despite their relative rarity, the NCLs represent a major cause of childhood- 
onset dementia, particularly in the European and Northern-American countries, where the incidence may be as high as 1:12.500 live births. ${ }^{11}$

The NCLs were historically divided in four main types, depending on age at onset: infantile NCL, late-infantile NCL, juvenile NCL and adult NCL. ${ }^{3}$ Even with the increasing number of underlying genes being identified, this historical classification still fits most - classical - patients: most patients with mutations in CLN1 display (classical) infantile NCL; most patients with mutations in CLN2 display (classical) late-infantile NCL and most patient with mutations in CLN3 display (classical) juvenile NCL. With the genetic unraveling of most NCLs, we came to understand why some patients - reported to display a "variant" NCL subtype - did not fit this classification. They for instance displayed a juvenile NCL phenotype, but with an earlier and more pronounced neurological involvement, and more importantly, a different accumulation picture than generally seen in juvenile NCL (granular osmiophilic deposits (GRODs) rather than fingerprint profiles (FPPs), and without vacuolated lymphocytes). ${ }^{12}$ With the identification of their genetic origin, we learned that these variant patients either had relatively mild mutations in a classical CLN gene causing a more attenuated phenotype, or had mutations in new CLN genes. ${ }^{13,14}$ Since then, the NCLs are rather being classified according to their genetic underpinning. ${ }^{15}$ Currently, up to 14 different NCL genes are recognized. Hence, it is essential to report the genetic background to avoid confusion. Table 1 aims to provide clarity into the NCL nomenclature. 
TABLE 1 | OVERVIEW OF THE NEURONAL CEROID LIPOFUSCINOSES

\begin{tabular}{|c|c|c|c|}
\hline Gene & Protein & Phenotype(s) & Old nomenclature \\
\hline \multirow[t]{4}{*}{$\begin{array}{l}\text { CLN1 } \\
\text { (PTT1) }\end{array}$} & $\begin{array}{l}\text { palmitoyl-protein } \\
\text { thioesterase } 1\end{array}$ & CLN1 disease, infantile & $\begin{array}{l}\text { Haltia-Santiavuori } \\
\text { disease }\end{array}$ \\
\hline & & $\begin{array}{l}\text { CLN1 disease, late } \\
\text { infantile }\end{array}$ & \\
\hline & & CLN1 disease, juvenile & \\
\hline & & CLN1 disease, adult & \\
\hline \multirow[t]{4}{*}{$\begin{array}{l}\text { CLN2 } \\
\text { (TPP2) }\end{array}$} & $\begin{array}{l}\text { tripeptidyl } \\
\text { peptidase } 1\end{array}$ & $\begin{array}{l}\text { CLN2 disease, late } \\
\text { infantile }\end{array}$ & $\begin{array}{l}\text { Jansky- } \\
\text { Bielschowsky } \\
\text { disease }\end{array}$ \\
\hline & & CLN2 disease, infantile & \\
\hline & & CLN2 disease, juvenile & \\
\hline & & $\mathrm{SCAR} 7^{16}$ & \\
\hline \multirow[t]{2}{*}{ CLN3 } & CLN3/ Battenin & CLN3 disease, juvenile & $\begin{array}{l}\text { Batten Disease } \\
\text { Batten-Spielmeyer- } \\
\text { Vogt } \\
\text { Spielmeyer-Sjogren }\end{array}$ \\
\hline & & $\begin{array}{l}\text { CLN3-associated retinal } \\
\text { degeneration }{ }^{17,18}\end{array}$ & \\
\hline $\begin{array}{l}\text { CLN4 } \\
\text { (DNAJC5) }\end{array}$ & $\begin{array}{l}\text { DnaJ heat shock } \\
\text { protein family } \\
\text { (Hsp40) member C5 }\end{array}$ & $\begin{array}{l}\text { CLN4 disease, adult } \\
\text { autosomal dominant }{ }^{19}\end{array}$ & Parry disease \\
\hline \multirow[t]{3}{*}{ CLN5 } & CLN5 & $\begin{array}{l}\text { CLN5 disease, late } \\
\text { infantile }^{20}\end{array}$ & \\
\hline & & CLN5 disease, juvenile & \\
\hline & & CLN5 disease, adult & \\
\hline \multirow[t]{2}{*}{ CLN6 } & CLN6 & $\begin{array}{l}\text { CLN6 disease, late } \\
\text { infantile }\end{array}$ & \\
\hline & & CLN6 disease, adult ${ }^{21,22}$ & Kufs disease, type A \\
\hline $\begin{array}{l}\text { CLN7 } \\
\text { (MFSD8) }\end{array}$ & $\begin{array}{l}\text { major facilitator } \\
\text { superfamily domain } \\
\text { containing } 8\end{array}$ & $\begin{array}{l}\text { CLN7 disease, late } \\
\text { infantile }^{23}\end{array}$ & \\
\hline \multirow[t]{3}{*}{ CLN8 } & CLN8 & $\begin{array}{l}\text { CLN8 disease, late } \\
\text { infantile }\end{array}$ & \\
\hline & & CLN8 disease, juvenile & \\
\hline & & CLN8 disease, EPMR ${ }^{24}$ & \\
\hline $\begin{array}{l}\text { CLN9-not } \\
\text { identified }\end{array}$ & unknown & & \\
\hline
\end{tabular}


TABLE 1 | CONTINUED

\begin{tabular}{|c|c|c|c|}
\hline Gene & Protein & Phenotype(s) & Old nomenclature \\
\hline \multirow[t]{4}{*}{$\begin{array}{l}\text { CLN10 } \\
\text { (CTSD) }\end{array}$} & cathepsin D & $\begin{array}{l}\text { CLN10 disease, } \\
\text { congenital }^{25}\end{array}$ & \\
\hline & & $\begin{array}{l}\text { CLN10 disease, late } \\
\text { infantile }\end{array}$ & \\
\hline & & CLN10 disease, juvenile & \\
\hline & & CLN10 disease, adult & \\
\hline \multirow{2}{*}{$\begin{array}{l}\text { CLN11 } \\
(G R N)\end{array}$} & granulin precursor & CLN11 disease, adult ${ }^{26}$ & \\
\hline & & $\begin{array}{l}\text { Frontotemporal } \\
\text { dementia }\end{array}$ & \\
\hline \multirow{2}{*}{$\begin{array}{l}\text { CLN12 } \\
\text { (ATP13A2) }\end{array}$} & ATPase cation & CLN12 disease, juvenile ${ }^{27}$ & \\
\hline & transporting $13 \mathrm{~A} 2$ & Kufor-Rakeb syndrome & \\
\hline $\begin{array}{l}\text { CLN13 } \\
\text { (CTSF) }\end{array}$ & cathepsin F & CLN13 disease, adult ${ }^{28}$ & Kufs disease type $B$ \\
\hline \multirow{2}{*}{$\begin{array}{l}\text { CLN14 } \\
(\text { KCTD7) }\end{array}$} & potassium channel & CLN14 disease, infantile ${ }^{29}$ & \\
\hline & $\begin{array}{l}\text { tetramerization } \\
\text { domain containing } 7\end{array}$ & $\begin{array}{l}\text { Progressive myoclonic } \\
\text { epilepsy }\end{array}$ & \\
\hline $\begin{array}{l}\text { "CLN15" } \\
(\text { TBCK) }\end{array}$ & $\begin{array}{l}\text { TBC1 domain- } \\
\text { containing kinase }\end{array}$ & $\begin{array}{l}\text { Non-progressive } \\
\text { neurological disease } \\
\text { with dysmorphic } \\
\text { features }{ }^{\star 30}\end{array}$ & \\
\hline
\end{tabular}

Bold = (classical) phenotype assumed to be caused by complete loss of protein function

* consensus has yet to be established to define whether this actually represents an NCL subtype as the non-progressive neurological disease with dysmorphic features argues against classification as an NCL.

Of note, while the term Batten disease was originally used to refer to juvenile $N C L$ only - most often caused by mutations in CLN3 - it is increasingly being used to refer to other NCLs or NCL in general. In this thesis, Batten disease refers to genetically defined CLN3 disease unless otherwise noted.

\section{CLN3 DISEASE}

CLN3 disease (OMIM \#204200) represents the most common of the NCLs and is the main focus of this thesis. Its classical disease course generally starts with a dramatically rapid loss of vision. ${ }^{31}$ Around 5-8 years of age, affected children may within short time fail to see the blackboard, but may also have 
new, and increasing, difficulties with color vision and night vision. Within a few months, their visual acuity may have already declined to around $10-20 \%$ at first presentation at the ophthalmologist. ${ }^{31}$ Within the next few years, affected children become blind. In parallel, the initially (sometimes) subtle learning and/or behavioral problems ${ }^{32}$ progress to more obvious signs of neurodegeneration, including the onset of seizures, dementia, and a Parkinson-like movement disorder. Most patients with classical CLN3 disease eventually die between their late teens and early twenties. ${ }^{33}$ Some patients display a more protracted phenotype with a retinal phenotype similar to that of classical CLN3 disease, but with a delayed onset of neurodegeneration. Still, the general paradigm was that CLN3 disease would - sooner or later - always include neurodegeneration. However, the identification of patients with a retina-only disease, who also have bi-allelic mutations in $C L N 3$, suggests that this view should be revised. ${ }^{17,18}$

\section{THE RETINA}

In any case, CLN3 disease affects the retina. Two main anatomical landmarks of the retina relevant for CLN3 disease are the optic disc and the macula. The retinal ganglion cell axons and glial cells form the optic nerve; the optic disc represents the beginning of the optic nerve and the point of exit for ganglion cell axons leaving the eye. The optic disc is visible in the back of the eye. Because locally no photoreceptors are present, we cannot see with that specific part of the retina. This is why the optic disc is also referred to as the blind spot. We however do not have a 'hole' in our view. This is because the missing information from the blind spot in the one eye is supplemented with the information from the other eye and vice versa. This blind spot caused by the optic disc is physiological, however, blind spots ("scotoma's") may also result from retinal or optic nerve diseases.

When you look at something, you obviously do not focus with your blind spot, but rather with the center of your retina: the macula lutea or "yellow spot". The macula lutea is responsible for the central, high-resolution, color vision that is achievable in good light. The macula, particularly in its central part the fovea centralis - contains the highest cone density of the retina. We have three types of cones: $\mathrm{S}, \mathrm{M}$ and $\mathrm{L}$, which respond to the different wavelengths, short (blue), medium (green), and long (red), respectively. Together, the 
cones perceive a continuous range of colors from deep red to violet. Color "blindness" is relatively common in the general population. About $8 \%$ of men are color blind (including those in the Kuper/Hoekstra-family). ${ }^{34}$ Most of them have an impaired function of the deutan (green) photoreceptors causing deuteranomalia or deuteranopia, commonly referred to as 'red-green color blindness'. Such congenital color blindness is stable over time, restricted to a specific color spectrum, and accompanied by good visual acuity. Only a small percentage of the color blind are literally color blind due to a disorder called 'achromatopsia'. These patients have no functioning cones at all and rely completely on their rods for their vision (see below). ${ }^{35}$ In Batten disease, patients develop color vision deficiency, due to their impaired cone function. The color vision deficiency in Batten disease worsens with progression of the retinal dystrophy.

The second type of photoreceptors are the rods. Rods are concentrated at the outer edges of the retina and are used for peripheral vision. Rods function best in dim light and are more sensitive than cone cells. They allow us to have vision in the dark and pick up peripheral movement. However, they are unable to discriminate color (hence the absence of color vision when you see in the dark) and do not have a high resolution (hence you still need to look towards what moves into your field of vision to clearly see it). Rods alone can provide a visual acuity of up to 0.1 , which indeed corresponds to the visual acuity associated with achromatopsia. ${ }^{35}$ In Batten disease, the cone impairment initially stands at the forefront of the visual phenotype. That is why patients in the early phase of the disease seem to 'look over' what they want to focus on since they rely on their peripheral vision for that. However, rod related abnormalities also occur early (for example the difficulties seeing in the dark). 
FIGURE 1 | RETINAL ANATOMY IN THE HEALTHY SITUATION COMPARED TO (EARLY) IN CLN3 DISEASE

Healthy individual
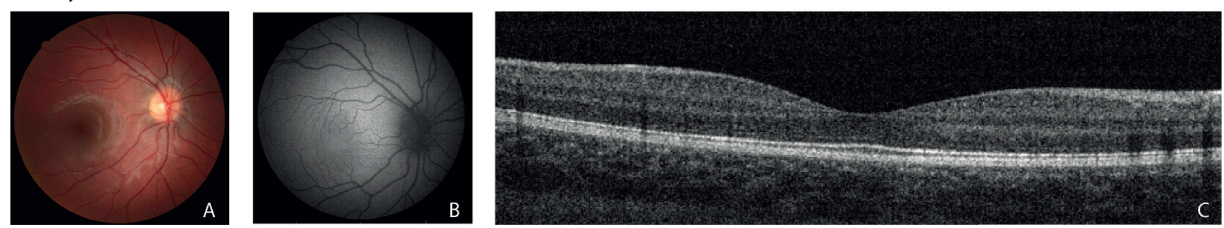

CLN3 disease
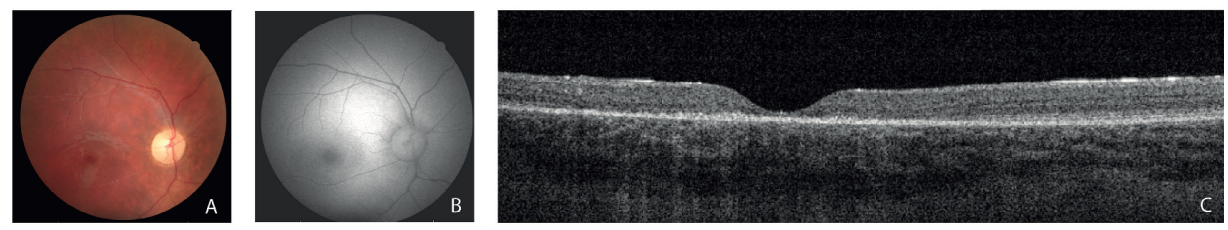

Representative fundoscopy (A), fundus autofluorescence (FAF) (B), and optical coherence tomography $(O C T)(C)$, from a healthy child compared to a CLN3 disease patient around diagnosis.

\section{ADVANCED OPHTHALMOLOGICAL TESTING}

When examining a patient with vision loss, in the case of CLN3 disease mostly children, the ophthalmologist can perform several advanced ophthalmological examinations. A subset of these, relevant for CLN3 disease, will be introduced below.

First, several imaging techniques can be used to visualize retinal anatomy. Fundoscopy, often performed with dilated pupils to achieve a more complete view of the retina, allows to examine the retinal surface. This can be done by hand, but also by taking a photograph of the fundus. On this image, abnormalities in the macula (e.g. a bull's eye maculopathy), abnormalities around the macula (e.g. pigmentary changes), abnormalities of the vessels (e.g. attenuated vessels), and abnormalities of the optic disc (e.g. optic disc pallor), indicative of a retinal dystrophy can be identified. However, any abnormalities can often be subtle and difficult to see at first, especially when the child does not cooperate. With fundus autofluorescence, abnormalities of the retina pigment epithelium (RPE) can be identified. ${ }^{36}$ It is a normal characteristic of several ocular structures to exhibit autofluorescence, i.e. they emit intrinsic fluorescence after stimulation by excitation energy. This 
autofluorescence derives from lipofuscin, predominantly resident in the RPE. The RPE is responsible for the turnover of photoreceptor outer segments. Lipofuscin accumulates as an oxidative by-product of this autophagic process. In CLN3 disease, the RPE contains less lipofuscin and thus shows less autofluorescence. ${ }^{37}$ Using optical coherence tomography (OCT), a picture of the cross section of the retina is made, allowing to assess the anatomy of the different retinal layers..$^{38}$ In Figure 1, the different retinal layers can be distinguished in the healthy retina. Relevant, particularly for Chapter 4 of this thesis is that the photoreceptors are shown in the 'grey' area in the outer retina. In the middle, at the 'dimple', resides the fovea centralis: here, the inner retinal layers are absent to ensure uninterrupted absorption of the light at the cones that are centered at the fovea.

Next, the function of the retina can be assessed by means of the electroretinogram (ERG). For the procedure, the pupils are dilated and Dawson, Trick, Litzkow (DTL) electrodes are placed near the cornea to measure the response of the retina to light. ${ }^{39}$ Using light stimuli of varying intensities in light or dark adapted conditions, the full field (ff)ERG assesses the function of the cones as well as the rods. When the macula only is atrophic, this has a great impact on vision since the cones are centered here. This may however not result in an aberrant ffERG since the macula covers only a small part of the whole surface of the retina. Therefore, a reduced cone ERG means that a disease is more widespread than the macula itself. If the rods are affected as well, abnormalities can also be seen on the dark adapted ffERG..$^{40}$ Following a stimulus, the ERG normally shows two waves (Figure 2). First, the negative a-wave, caused by hyperpolarization of the photoreceptors and next the positive b-wave, caused by the activity of the 'conduction' layers, that is the bipolar cells, the inner plexiform layer and the Muller cells. Thus, the a-wave should form a dip, the b-wave should form a peak. Responses are judged on both axes with the $y$-axis representing the amplitude, and $x$-axis representing time (latency). Amplitude and latency differ depending on the intensity of the stimulus used, as set by the International Society of Clinical Electrophysiology of Vision (ISCEV). ${ }^{41}$ 


\section{FIGURE 2 | THE ELECTRORETINOGRAM}

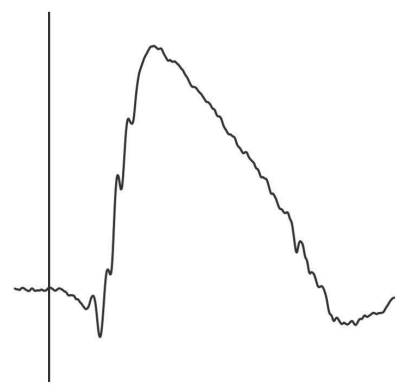

Schematic representation of the a-wave (dip) and the b-wave (peak) in the electroretinogram (ERG) of a healthy individual

In case of Batten disease, the ophthalmological examinations will make clear that the child suffers from a severe retinal dystrophy. Then, the underlying cause needs to be identified. Knowing the underlying cause as early as possible is essential to tailor counseling. However, Batten disease is often mistaken initially for an isolated retinal dystrophy (mostly Stargardt disease). ${ }^{31}$ This diagnostic challenge can cause frustration for both the child as well as their family and others close to them.

When (ultimately) referred to a tertiary metabolic center, the diagnosis of CLN3 disease can quickly be confirmed or excluded preliminary by assessing the presence of vacuolated lymphocytes. ${ }^{42,43}$ Genetic confirmation follows within a few weeks to months.

\section{CLN3}

\section{CLN3 GENE}

With the exception of the ultra-rare CLN4, all NCLs including CLN3 disease are inherited in an autosomal recessive manner. CLN3 resides at chromosomal location 16p12.1. ${ }^{44}$ Within CLN3, around 80 different disease causing mutations have been identified (recorded in the University College London NCL mutation database), although about $75 \%$ of patients are homozygous and about $20 \%$ are compound heterozygous for a single mutation: a deletion of 966 base pairs spanning exon 7 and 8 . This so-called 1 kilo base (kb) deletion most probably 
derives from a common European ancestor (founder mutation), explaining the higher incidence of CLN3 disease in people of European descent. ${ }^{44,45}$ As commonly seen in genetic disorders, the genotype correlates with phenotype strength: the $1 \mathrm{~kb}$ deletion is expected to severely affect the protein and to result in (near-)complete loss of function. Homozygote carriers of this mutation display the classical CLN3 disease phenotype. Several missense mutations with apparently less severe impact on protein function lead to protracted CLN3 disease or even CLN3-associated (isolated) retinal degeneration. ${ }^{17,18,45,46,47}$

\section{CLN3 PROTEIN}

CLN3 is conserved among vertebrate and non-vertebrate species, suggesting an evolutionary old function, but the exact nature of this function awaits elucidation. CLN3 is a highly hydrophobic protein of 438 amino acid residues with little to no homology to other proteins. It is thought to be a membrane protein, with a looped structure spanning the membrane multiple times. ${ }^{44}$ CLN3 most likely resides in lysosomal membranes but localization at other cell compartments has also been reported. These partly conflicting reports derive from the lack of specific antibodies and probably low expression levels. ${ }^{48}$

\section{STORAGE MATERIAL ACCUMULATION}

The characteristic accumulation of storage material - together with the clinical symptomatology - has historically formed the basis for the classification of the NCLs. In infantile NCL, the stored material appears as granular osmiophilic deposits (GRODs), in late-infantile NCLs as curvilinear profiles (CVPs), and in juvenile NCL as fingerprint profiles (FPPs) (Figure 3) whereas in other NCLs a mixture of these appearances is often seen. ${ }^{43}$ Early studies tried to elucidate a potential link between the CLN proteins and storage material, but they did not succeed. ${ }^{49,50}$ The current paradigm explains the storage material accumulation as a non-relevant result of lysosomal malfunctioning. ${ }^{51}$ Intriguingly however, the composition of the storage material is rather specific: in CLN1, CLN4 and CLN10 the storage material consists mainly of sphingolipid activator proteins (saposins) A and D. In the other NCLs - including CLN3 disease - the stored material consists mainly of the c-subunit of mitochondrial ATP synthase. ${ }^{49,50,52}$ It is currently poorly understood why these proteins specifically accumulate in the NCLS. 
FIGURE 3 | TYPICAL CLN3 DISEASE FINGERPRINT PROFILES AS VISIBLE AT THE LEVEL OF ELECTRON MICROSCOPY

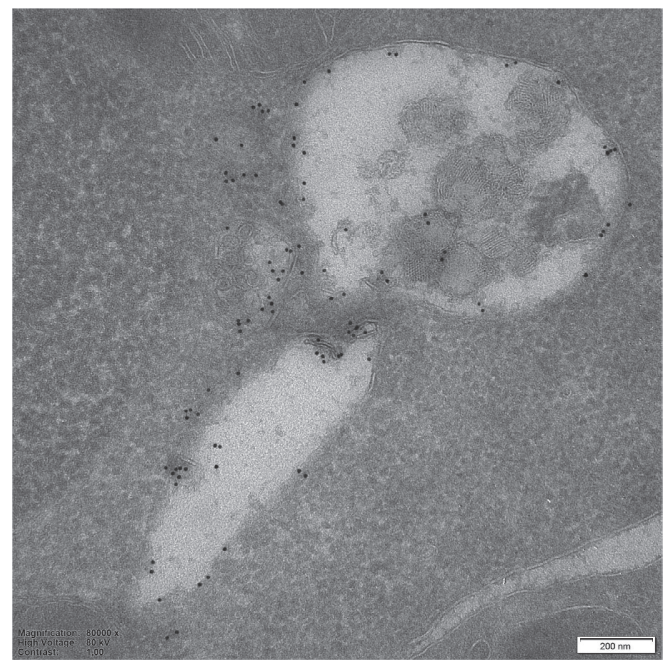

EM picture of a lymphocyte of a (classical) CLN3 disease patient. In the cytoplasm, large vacuoles, that are either empty, or include storage material displayed as fingerprint profiles can be identified. Black dots = staining for lysosomal associated membrane protein-1 (LAMP-1).

\section{LYMPHOCYTE VACUOLIZATION}

The GRODs, as well as the CVPs and FPPs are characteristic for the NCLs, but they are only visible on electron microscopy (EM). In CLN3 disease, however, characteristic vacuoles are formed in lymphocytes, which are visible on light microscopy (LM) (Figure 4). Although several other lysosomal storage disorders that also display (some) lymphocytic vacuolization have been described, it is a unique feature within the NCLs. ${ }^{42,43}$

Their visibility on LM makes the assessment of lymphocytic vacuoles suitable for daily clinical practice. In our center of expertise, we use the presence or absence of lymphocyte vacuolization to preliminary confirm or rule out the diagnosis of CLN3 disease, respectively. However, this manual assessment is generally not usable in general clinical practice as it requires expertise to distinguish vacuoles due to CLN3 disease from the occasional vacuoles due to infection that can also be present in healthy people. ${ }^{53}$ 
FIGURE 4 | ABUNDANT LYMPHOCYTE VACUOLIZATION IN (CLASSICAL) CLN3 DISEASE

healthy control

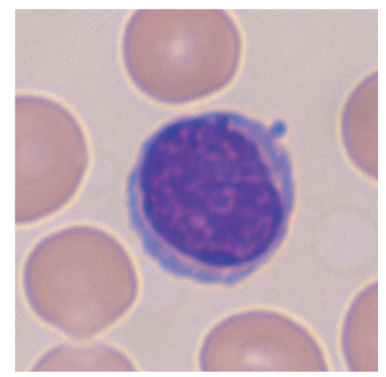

CLN3 disease

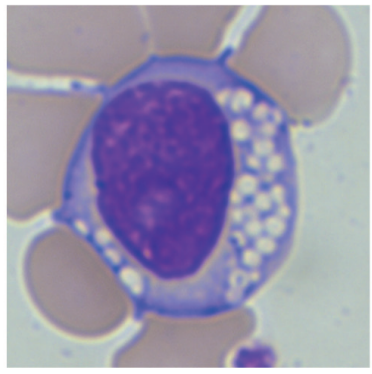

LM image of a lymphocyte of a healthy individual (left) compared to a lymphocyte of a patient with (classical) CLN3 disease (right) showing abundant vacuolization.

\section{BEYOND THE BLIND SPOTS}

Despite the passing of two centuries since the first description of patients suffering from CLN3 disease and despite the passing of decades since the identification of the responsible CLN3 gene, we do not yet understand how this genetic defect exactly leads to disease. Metaphorically, one could say that what connects the genetic fault to the disease, lays in our blind spot.

Saying that something lays in our blind spot implies that we fail to see something that is actually obvious. This hunch - that cues towards true understanding were already in front of us, yet invisible - was what started the research that is described in this thesis. We questioned several paradigms, focusing on the following three main aims of this thesis:

\section{AIMS OF THIS THESIS}

- (Part 1): To improve early recognition of CLN3 disease by delineating its early disease phase.

- (Part 2): To improve (clinical) understanding of CLN3 disease by delineating the nuances in its phenotypic spectrum.

- (Part 3): To improve (pathophysiological) understanding of CLN3 disease by delineating its characteristic storage material accumulation. 


\section{OUTLINE OF THIS THESIS}

This thesis focuses on elucidating the blind spots in our understanding of Batten disease (CLN3 disease) with the aim to ultimately pave the way for treatment options for this major cause of childhood dementia. To that aim, the thesis has been divided into three parts.

Part 1 deals with the early phase of CLN3 disease. Chapter 2 and 3 focus on the paradigm that in CLN3 disease, vision loss precedes neurodegeneration and show that the actual early onset of neurodegeneration has been a major blind spot of us. In Chapter 2 a comprehensive meta-analysis of published case reports is supplemented with data from our own cohort focused on the timing of cognitive decline in CLN3 disease. Chapter 3 expands this focus on the early neurodegeneration to the motor function impairment, using the 6 minute walk test (6MWT).

Despite its early onset, neurologic involvement is still subtle at disease onset which makes CLN3 disease difficult to recognize for the ophthalmologist whom the patients often meet first. Chapter 4 discusses several CLN3 diseasespecific ophthalmological characteristics, identified using a deep phenotyping approach that help the ophthalmologist to recognize CLN3 disease early.

In Part 2, two chapters discuss recent shifts in our old view of the CLN3 disease phenotypic spectrum. Chapter 5 discusses an unexpected protracted disease course in three patients with a mutation in the start codon of CLN3, initially presumed to be a severe mutation. Chapter 6 discusses an even milder phenotypic entity of CLN3 disease, CLN3-associated (isolated) retinal degeneration.

In Part 3, the attention is shifted from the clinical understanding towards pathophysiological understanding, with a special focus on storage material accumulation. In Chapter 7 , we quantify lymphocyte vacuolization -using both a manual and an automatic approach - to diagnose CLN3 disease and classify disease severity. In Chapter 8 , we revisit the storage material accumulation in the NCLs and hypothesize how the CLN proteins might function in either saposins $A$ and $D$ or subunit c turnover. 
As a comprehensive overview, Chapter 9 provides a critical discussion of the contents of this thesis. Particularly, applications and implications for diagnosis, follow-up, and therapy development and monitoring, are discussed. Finally, in Chapter 10 the previous chapters are summarized in both English and Dutch. 


\section{REFERENCES}

${ }^{1}$ Batten F. Cerebral degeneration with symmetrical changes in the maculae in two members of a family, Trans. Ophthalmol. Soc. U. K. 23 (1903) 386-390.

${ }^{2}$ Stengel C. Beretning om et mærkeligt Sygdomstilfælde hos fire Sødskende i Nærheden af Røraas, Eyr Med. Tidskr. 1 (1826) 347-352.

${ }^{3}$ Haltia M, Goebel HH. The neuronal ceroid-lipofuscinoses: a historical introduction. Biochim Biophys Acta. 2013 Nov;1832(11):1795-800.

${ }^{4}$ Zeman W, Dyken P. Neuronal ceroid-lipofuscinosis (Batten's disease): relationship to amaurotic family idiocy? Pediatrics. 1969 Oct;44(4):570-83.

${ }^{5}$ Isolation of a novel gene underlying Batten disease, CLN3. The International Batten Disease Consortium. Cell. 1995 Sep 22;82(6):949-57.

${ }^{6}$ Vesa J, Hellsten E, Verkruyse LA et al. Mutations in the palmitoyl protein thioesterase gene causing infantile neuronal ceroid lipofuscinosis. Nature. 1995;376(6541):584-587.

${ }^{7}$ Sleat DE, Donnelly RJ, Lackland $\mathrm{H}$ et al. Association of mutations in a lysosomal protein with classical late-infantile neuronal ceroid lipofuscinosis. Science. $1997 \mathrm{Sep}$ 19;277(5333):1802-5.

${ }^{8}$ Siintola E, Partanen S, Strömme P et al. Cathepsin D deficiency underlies congenital human neuronal ceroid-lipofuscinosis. Brain. 2006 Jun;129(Pt 6):1438-45.

${ }^{9}$ Nelvagal HR, Lange J, Takahashi K, Tarczyluk-Wells MA, Cooper JD. Pathomechanisms in the neuronal ceroid lipofuscinoses. Biochim Biophys Acta Mol Basis Dis. 2019 Oct 31:165570.

${ }^{10} \mathrm{Haltia}$ M. The neuronal ceroid-lipofuscinoses. J Neuropathol Exp Neurol. 2003 Jan;62(1):1-13.

${ }^{11}$ Geraets RD, Koh Sy, Hastings ML, Kielian T, Pearce DA, Weimer JM. Moving towards effective therapeutic strategies for Neuronal Ceroid Lipofuscinosis. Orphanet J Rare Dis. 2016 Apr 16;11:40.

${ }^{12}$ Aberg L, Järvelä I, Rapola J, Autti T, Kirveskari E, Lappi M, Sipilä L, Santavuori P. Atypical juvenile neuronal ceroid lipofuscinosis with granular osmiophilic deposit-like inclusions in the autonomic nerve cells of the gut wall. Acta Neuropathol. 1998 Mar;95(3):306-12.

${ }^{13}$ Wisniewski KE, Kaczmarski A, Kida E et al. Reevaluation of neuronal ceroid lipofuscinoses: atypical juvenile onset may be the result of CLN2 mutations. Mol Genet Metab. 1999 Apr;66(4):248-52.

${ }^{14}$ Philippart M, Mena I, Wisniewski KE, Pineda G. Impaired temporo-occipital blood flow in an atypical CLN1 case with late infantile onset and granular osmiophilic deposits. Eur J Paediatr Neurol. 2001;5 Suppl A:185-7.

${ }^{15}$ Williams RE, Mole SE. New nomenclature and classification scheme for the neuronal ceroid lipofuscinoses. Neurology. 2012;79:183-191.

${ }^{16}$ Sun Y, Almomani R, Breedveld GJ et al. Autosomal recessive spinocerebellar ataxia 7 (SCAR7) is caused by variants in TPP1, the gene involved in classic late-infantile neuronal ceroid lipofuscinosis 2 disease (CLN2 disease). Hum Mutat. 2013; 34:706-713.

${ }^{17}$ Wang F, Wang H, Tuan HF et al. Next generation sequencing-based molecular diagnosis of retinitis pigmentosa: identification of a novel genotype-phenotype correlation and clinical refinements. Hum Genet. 2014 Mar;133(3):331-45. doi: 10.1007/s00439-013-13815. Epub 2013 Oct 24.

${ }^{18} \mathrm{Ku}$ CA, Hull S, Arno G et al. Detailed Clinical Phenotype and Molecular Genetic Findings in CLN3-Associated Isolated Retinal Degeneration. JAMA Ophthalmol. 2017 Jul 1;135(7):749760. 
${ }^{19}$ Velinov M, Dolzhanskaya N, Gonzalez M et al. Mutations in the gene DNAJC5 cause autosomal dominant Kufs disease in a proportion of cases: study of the Parry family and 8 other families. PLoS One. 2012;7(1):e29729.

${ }^{20}$ Savukoski M, Klockars T, Holmberg V, Santavuori P, Lander ES, Peltonen L. CLN5, a novel gene encoding a putative transmembrane protein mutated in Finnish variant late infantile neuronal ceroid lipofuscinosis. Nat Genet. 1998; 19:286-288.

${ }^{21}$ Wheeler RB, Sharp JD, Schultz RA, Joslin JM, Williams RE, Mole SE. The gene mutated in variant late infantile neuronal ceroid lipofuscinosis (CLN6) and nclf mutant mice encodes a novel predicted transmembrane protein. Am J Hum Genet. 2002; 70:537-542.

${ }^{22}$ Arsov T, Smith KR, Damiano J et al. Kufs disease, the major adult form of neuronal ceroid lipofuscinosis, caused by mutations in CLN6. Am J Hum Genet. 2011; 88:566-573.

${ }^{23}$ Siintola E, Topcu M, Aula N et al. The novel neuronal ceroid lipofusinosis gene MFSD8 encodes a putative lysosomal transporter. Am J Hum Genet. 2007; 81:136-146.

${ }^{24}$ Ranta S, Zhang Y, Ross B et al. The neuronal ceroid lipofuscinoses in human EPMR and mnd mutant mice are associated with mutations in CLN8. Nat Genet. 1999; 23:233-236.

${ }^{25}$ Siintola E, Partanen S, Strömme P et al. Cathepsin D deficiency underlies congenital human neuronal ceroid lipofuscinosis. Brain. 2006; 129:1438-1445.

${ }^{26}$ Smith KR, Damiano J, Franceschetti S et al. Strikingly different clinicopathological phenotypes determined by progranulin-mutation dosage. Am J Hum Genet. 2012; 90:1102-1107.

${ }^{27}$ Bras J, Verloes A, Schneider SA, Mole SE, Guerreiro RJ. Mutation of the parkinsonism gene ATP13A2 causes neuronal ceroid-lipofuscinosis. Hum Mol Genet. 2012; 21:2646-2650.

${ }^{28}$ Smith KR, Dahl HH, Canafoglia L et al. Cathepsin F mutations cause Type B Kufs disease, an adult-onset neuronal ceroid lipofuscinosis. Hum Mol Genet. 2013 Apr 1;22(7):1417-23.

${ }^{29}$ Staropoli JF, Karaa A, Lim ET et al. A homozygous mutation in KCTD7 links neuronal ceroid lipofuscinosis to the ubiquitin-proteasome system. Am J Hum Genet. $2012 \mathrm{Jul}$ 13;91(1):202-8.

${ }^{30}$ Beck-Wödl S, Harzer K, Sturm M et al. Homozygous TBC1 domain-containing kinase (TBCK) mutation causes a novel lysosomal storage disease - a new type of neuronal ceroid lipofuscinosis (CLN15)? Acta Neuropathol Commun. 2018 Dec 27;6(1):145.

${ }^{31}$ Collins J, Holder GE, Herbert H, Adams GG. Batten disease: features to facilitate early diagnosis. Br J Ophthalmol. 2006 Sep;90(9):1119-24.

${ }^{32}$ Spalton DJ, Taylor DSI, Sanders MD (1980) Juvenile Batten's disease: an ophthalmological assessment of 26 patients. Br J Ophthalmol 64: 726-732.

${ }^{33}$ Kousi M, Lehesjoki AE, Mole SE. Update of the mutation spectrum and clinical correlations of over 360 mutations in eight genes that underlie the neuronal ceroid lipofuscinoses. Hum Mutat. 2012 Jan;33(1):42-63.

${ }^{34}$ Simunovic MP. Colour vision deficiency. Eye (Lond). 2010;24(5):747-55. doi: 10.1038/ eye.2009.251. Epub 2009 Nov 20.

${ }^{35}$ Kohl S, Varsanyi B, Antunes GA et al. CNGB3 mutations account for $50 \%$ of all cases with autosomal recessive achromatopsia. Eur J Hum Genet. 2005 Mar;13(3):302-8.

${ }^{36}$ Schmitz-Valckenberg S, Holz FG, Bird AC, Spaide RF. Fundus autofluorescence imaging: review and perspectives. Retina. 2008 Mar;28(3):385-409.

${ }^{37}$ Wright GA, Georgiou M, Robson AG et al. Juvenile Batten Disease (CLN3): Detailed Ocular Phenotype, Novel Observations, Delayed Diagnosis, Masquerades, and Prospects for Therapy [published online ahead of print, 2019 Nov 13]. Ophthalmol Retina. 2019;S24686530(19)30629-3. 
${ }^{38}$ Hee MR, Izatt JA, Swanson EA et al. Optical coherence tomography of the human retina. Arch Ophthalmol. 1995 Mar;113(3):325-32.

${ }^{39}$ Dawson WW, Trick GL, Litzkow CA. Improved electrode for electroretinography. Invest Ophthalmol Vis Sci 1979 18:988-991.

${ }^{40}$ Weleber RG. The dystrophic retina in multisystem disorders: the electroretinogram in neuronal ceroid lipofuscinoses. Eye (Lond). 1998;12 ( Pt 3b):580-90. Review.

${ }^{41}$ McCulloch DL, Marmor MF, Brigell MG et al. ISCEV standard for full-field clinical electroretinography (2015 update). Doc Ophthalmol 2015;130(1):1-12.

${ }^{42}$ Anderson G, Smith VV, Malone M, Sebire NJ. Blood film examination for vacuolated lymphocytes in the diagnosis of metabolic disorders; retrospective experience of more than 2,500 cases from a single centre. J Clin Pathol. 2005 Dec;58(12):1305-10.

${ }^{43}$ Anderson GW, Goebel HH, Simonati A et al. Human pathology in NCL. Biochim Biophys Acta. 2013 Nov;1832(11):1807-26.

${ }^{44}$ Mirza M, Vainshtein A, DiRonza A et al. The CLN3 gene and protein: What we know. Mol Genet Genomic Med. 2019 Dec;7(12):e859.

${ }^{45}$ Kousi M, Lehesjoki AE, Mole SE. Update of the mutation spectrum and clinical correlations of over 360 mutations in eight genes that underlie the neuronal ceroid lipofuscinoses. Hum Mutat. 2012 Jan;33(1):42-63.

${ }^{46}$ Mole SE, Williams RE, Goebel HH. Correlations between genotype, ultrastructural morphology and clinical phenotype in the neuronal ceroid lipofuscinoses. Neurogenetics, 6 (2005), pp. 107-126.

${ }^{47}$ Munroe PB, Mitchison HM, O'Rawe AM et al. Spectrum of mutations in the Batten disease gene, CLN3. Am J Hum Genet. 1997 Aug;61(2):310-6.

${ }^{48}$ Nelson T, Pearce DA, Kovács AD. Lack of specificity of antibodies raised against CLN3, the lysosomal/endosomal transmembrane protein mutated in juvenile Batten disease. Biosci Rep. 2017 Nov 23;37(6).

${ }^{49}$ Palmer DN, Fearnley IM, Walker JE, Hall NA, Lake BD, Wolfe LS, Haltia M, Martinus RD, Jolly RD (1992) Mitochondrial ATP synthase subunit c storage in the ceroid-lipofuscinoses (Batten disease). Am J Med Genet 42 : 561-567.

${ }^{50}$ Palmer DN, Bayliss SL, Westlake VJ (1995) Batten disease and the ATP synthase subunit c turnover pathway: raising antibodies to subunit c. Am J Med Genet $57: 260-265$.

${ }^{51}$ Palmer DN. The relevance of the storage of subunit c of ATP synthase in different forms and models of Batten disease (NCLs). Biochim Biophys Acta. 2015 Oct;1852(10 Pt B):2287-91.

${ }^{52}$ Tyynela J, Palmer DN, Baumann M, Haltia M. Storage of saposins A and D in infantile neuronal ceroid-lipofuscinosis. FEBS Lett 330, 8-12 (1993).

${ }^{53}$ Simon MW. The atypical lymphocyte. International Pediatrics. 2003; 18(1):20-22. 



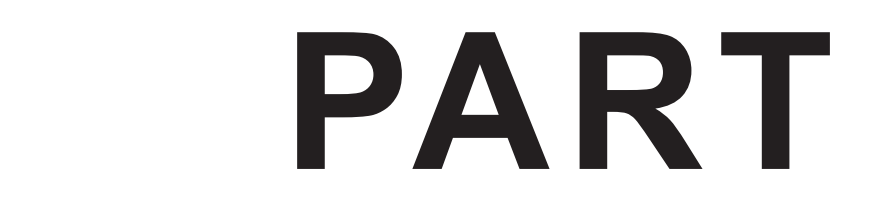

EARLY RECOGNITION OF CLN3 DISEASE

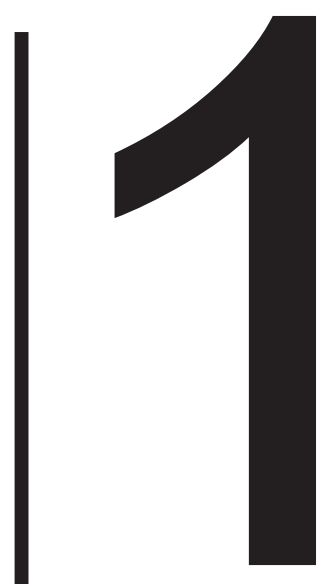





\section{CHAPTER TIMING OF COGNITIVE DECLINE IN CLN3 DISEASE}

Willemijn F.E. Kuper, Claudia van Alfen, Roeliene H. Rigterink, Sabine A. Fuchs, Maria M. van Genderen, Peter M. van Hasselt J Inherit Metab Dis. 2018 Mar;41(2):257-261

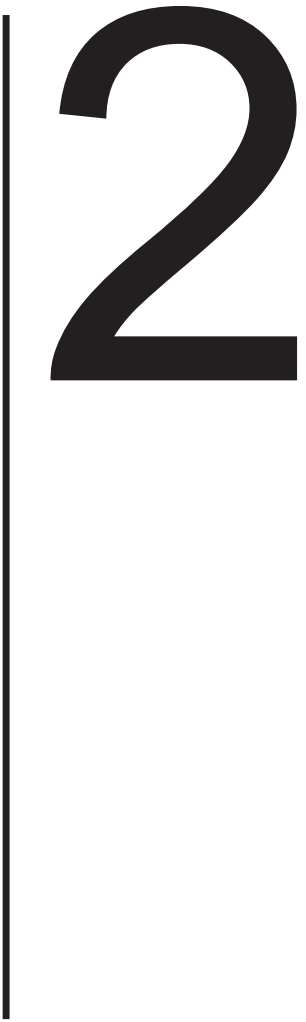




\section{ABSTRACT}

Background: CLN3 disease is a major cause of childhood neurodegeneration. Onset of visual failure around 6 years of age is thought to precede cognitive deterioration by a few years, but casuistic reports question this paradigm. The aim of our study is to delineate timing of cognitive decline in CLN3 disease.

Methods: Early neurocognitive functioning in CLN3 disease was analyzed using age at onset of visual and cognitive decline and IQ scores from literaturederived patient descriptions, supplemented with IQ scores and school history from a retrospective referral center cohort. We analyzed protracted and classical CLN3 separately and added a control group of patients diagnosed with juvenile onset macular degeneration (early onset Stargardt disease) to control for possible effects of rapid vision loss on neurocognitive functioning.

Results: Onset of cognitive decline at a mean age of 6.8 years (range 2-13 years, $\mathrm{n}=19$ ) paralleled onset of visual deterioration at a mean age of 6.4 years (range 4-9 years, $n=81$ ) as supported by an early decline in IQ scores in classical CLN3 disease. Onset and course of vision loss was similar in patients with protracted CLN3. The decreased IQ levels at diagnosis (mean 68.4, range 57-79, $n=9$ ) in the referral cohort were consistently associated with an aberrant early school history contrasting normal school history and cognition in Stargardt disease patients.

Conclusions: Cognitive dysfunction is universally present around diagnosis in classical CLN3 disease.

Keywords: CLN3 disease; Childhood neurodegeneration; Natural history; Neuronal ceroid lipofuscinosis (NCL) 


\section{INTRODUCTION}

CLN3 disease (OMIM \#204200), also known as juvenile neuronal ceroid lipofuscinosis or Batten disease, is the most frequent of the neuronal ceroid lipofuscinoses (NCLs). These genetically and clinically heterogeneous degenerative disorders of the brain and, in most forms, of the retina are unified by the intracellular accumulation of auto fluorescent storage material in most tissues. Collectively, they comprise a major cause of childhood neurodegeneration (Haltia 2003; Kousi et al. 2012).

Patients with CLN3 disease present with rapid visual decline around 6 years of age. It is generally believed that cognitive deterioration does not occur until several years after presentation (Kousi et al. 2012). Indeed, cognitive decline may not be apparent until well into adulthood in the protracted form of CLN3 disease displayed by a minority of patients (Lauronen et al. 1999; Munroe et al. 1997). Some casuistic reports, however, have suggested the presence of neuropsychological problems around, or even before, the onset of visual deterioration in the common classical phenotype (Kristensen and Lou 1983; Lamminranta et al. 2001; Spalton et al. 1980). It is presently unclear whether these problems should be attributed to the rapid vision loss or reflect early neurodegeneration.

To delineate timing of neurocognitive decline in CLN3 disease, we performed a systematic literature search and included the retrieved cases in a metaanalysis of case reports. Subsequently, we corroborated the data from the literature search with a cohort of patients diagnosed with CLN3 disease from the Dutch national NCL referral center and - to delineate the influence of rapid vision loss - we compared these to a cohort of patients with isolated retinal degeneration due to Stargardt disease.

\section{METHODS}

\section{LITERATURE SEARCH}

We searched PubMed and Embase for individual patients published until June 2017 with the search terms "CLN3 disease", "juvenile neuronal ceroid lipofuscinosis", “JNCL”, “juvenile NCL”, "batten disease”, "batten's disease” "CLN3", "NCL3", "spielmeyer vogt sjogren", "batten spielmeyer vogt", "spielmeyer vogt", "spielmeyer sjogren" and "juvenile amaurotic idiocy". We 
manually checked the reference lists of included articles to identify additional suitable studies.

Patients were considered for inclusion if diagnosis of CLN3 disease was confirmed. Both genetic analysis confirming biallelic variants in CLN3 and blood smear analysis revealing lymphocyte vacuolization, which is known to discriminate CLN3 disease from other NCL types, were considered sufficient proof (Anderson et al. 2013). Secondly, sufficient clinical data had to be retrievable (at least one out of: age at onset of visual failure, age at onset of cognitive decline, IQ test results). To avoid double inclusion of patients we matched patients if possible with their patient IDs from the UCL CLN3 mutation and patient databases (http://www.ucl.ac.uk/ncl/cln3.shtml) and we manually checked case reports on demographic and clinical characteristics. Patients displaying classical CLN3 disease were analyzed separately from a small subset displaying protracted CLN3 disease primarily based on genotype (Kousi et al. 2012; Munroe et al. 1997). If the genotype was unknown, patients were classified as classical unless the author explicitly classified the disease course as protracted.

For analysis, we extracted age at onset of visual failure, age at onset of cognitive decline, and IQ test results.

\section{REFERRAL CENTER COHORT}

To corroborate the findings from literature, we analyzed neurocognitive functioning around diagnosis in a referral center cohort of patients diagnosed from 1987 to 2016 with classical CLN3 disease. To control for possible effects of rapid early vision loss on neurocognitive functioning, we created a cohort of patients diagnosed with early onset Stargardt disease (OMIM \#248200). Early onset Stargardt disease, presenting between 5 and 10 years, is associated with a similarly rapid loss of vision and was therefore considered a valid control group (Lambertus et al. 2015).

For data analysis, we extracted results from IQ tests performed around diagnosis and school history prior to and after diagnosis. 


\section{STANDARD PROTOCOL APPROVALS, REGISTRATIONS, AND PATIENT CONSENTS}

A waiver of requirement for ethical review was granted by the Medical Ethical Committee at the University Medical Center Utrecht, the Netherlands.

\section{DATA ANALYSIS}

Kaplan-Meier curves were used to analyze age at onset of visual and cognitive deterioration in patients with either classical or protracted CLN3 disease. The mean was provided if the data were normally distributed; otherwise, the median was provided. Analysis of statistically significant differences in survival curves was performed using the log-rank test. Analysis of IQ scores was performed using Pearson $r$ correlation for the overall decline and the paired t-test for the decline in IQ scores per patient. A p-value of $<0.05$ was considered significant. Prism software (version 7.02, Graphpad Software, San Diego, CA) was used for statistical analysis.

\section{RESULTS}

The literature search revealed 36 studies, describing 104 classical and 14 protracted CLN3 disease patients, that were eligible for inclusion (Suppl. Tables 1 and 2).

\section{TIMING OF COGNITIVE DECLINE}

Onset of cognitive decline at a mean age of 6.8 years (range 2-13 years, $n=19$ ) paralleled onset of visual deterioration (mean age of 6.4 years, range 4-9 years, $\mathrm{n}=81$ ) in patients with classical CLN3 disease (Fig. 1). This finding remained when analysis was restricted to patients with genetically confirmed classical CLN3 disease $(n=73)$ (mean age at onset of visual deterioration $(n=50)$ and cognitive decline ( $n=14): 6.2$ years and 7.1 years, respectively). Onset of vision loss was only slightly later in protracted CLN3 disease (Fig. 2; mean age at onset 7.1 years, range $5-10$ years, $n=9$ ) similarly resulting in blindness within a few years after onset (data not shown) while cognition was unaffected around diagnosis and remained normal until adulthood. 


\section{FIGURE 1}

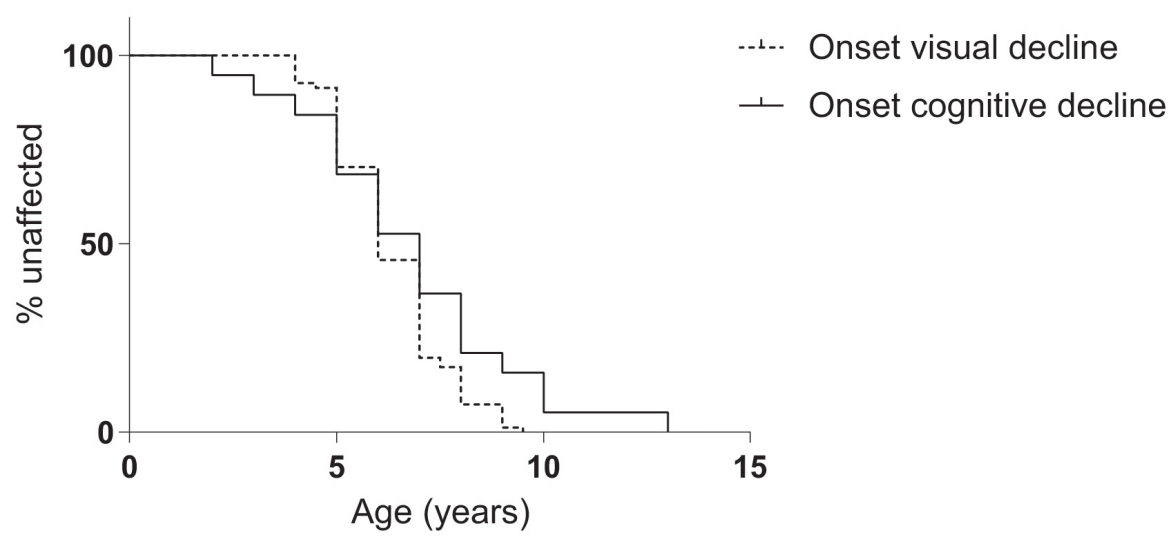

Onset of visual deterioration versus onset of cognitive decline in classical CLN3 disease. Kaplan-Meier survival curves of onset of cognitive decline $(n=19)$ versus onset of visual deterioration $(n=81)$ in classical CLN3 disease $(p=0.06)$

\section{FIGURE 2}

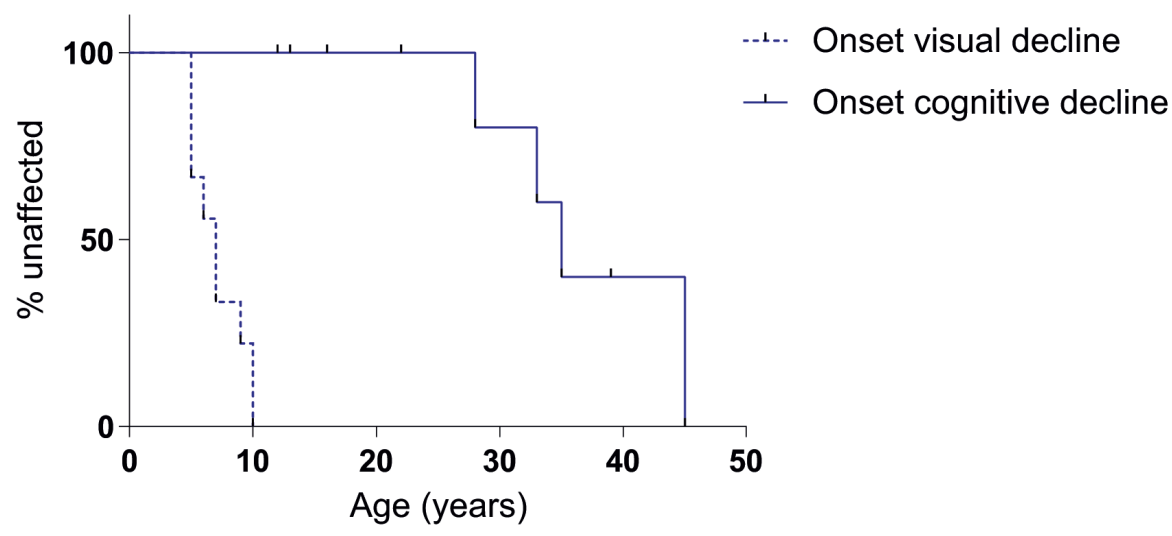

Onset of visual deterioration versus onset of cognitive decline in protracted CLN3 disease. Kaplan-Meier survival curves of onset of cognitive decline ( $n=10$ including censored cases) versus onset of visual deterioration $(n=9)$ in protracted CLN3 disease $(p<0.001)$ 
In patients with classical CLN3 disease, IQ scores declined from 91.4 (range $84-105, n=7$ ) at 6 years of age to 69.3 (range $53-80, n=4$ ) at 9 years of age $(r-0.5596 ; p 0.005)$, in line with the overall profound inverse correlation of IQ scores with age ( $n=52$ scores from 25 patients, $r-0.8239, p<0.001)$, contrasting the normal intelligence scores up to adulthood in protracted CLN3 disease (Fig. 3). This decline was also observed upon paired analysis of first and last IQ measurements in individual patients (from a mean IQ of 85.8 (49-106) to a mean IQ of $70.8(40-88), p<0.001)$.

\section{FIGURE 3}

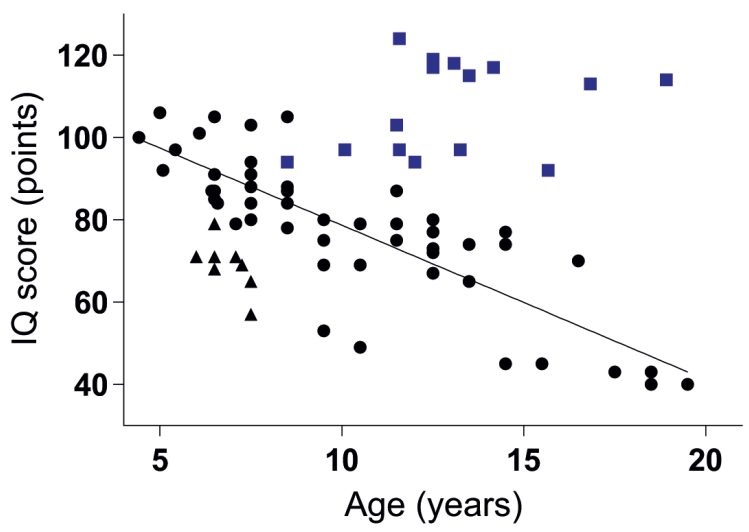

- classical CLN3

- protracted CLN3 classical CLN3 referall center

IQ scores in classical versus protracted CLN3 disease. IQ score decline in classical CLN3 disease versus IQ scores in protracted CLN3 disease. $r=-0.8239$ in classical CLN3 disease versus $r=0.3013$ in protracted CLN3 disease.

These data indicated that cognitive decline in patients with classical CLN3 disease sets in appreciably earlier than expected. However, small sample size and the significant amount of missing data precluded a more definite conclusion. To corroborate these findings we therefore evaluated the timing of cognitive decline in a separate cohort of 21 patients diagnosed with classical CLN3 disease from the Dutch national NCL referral center and - to delineate the influence of rapid vision loss - we compared these to a cohort of 13 patients with isolated retinal degeneration due to early onset Stargardt disease.

In this referral center cohort, IQ tests were performed around the time of diagnosis in 13 patients with classical CLN3 disease. In four patients the IQ 
tests could not be completed (due to aberrant behavior (1), attention deficit (1), unreliable, highly variable test results (1), and unspecified (1)). The IQ scores in the remaining nine patients were all well below average, in a range similar to, or even lower than, the range of patients derived from literature (mean 68.4, range 57-79, age 6-7 years) (Figs. 3 and and4).4). The decrease was more pronounced on performance than verbal subscales (Fig. (Fig.4).4). IQ tests around diagnosis in early onset Stargardt disease patients yielded normal results ( $n=4$ : IQ scores of $95,101,96$, and 100 , respectively).

\section{FIGURE 4}

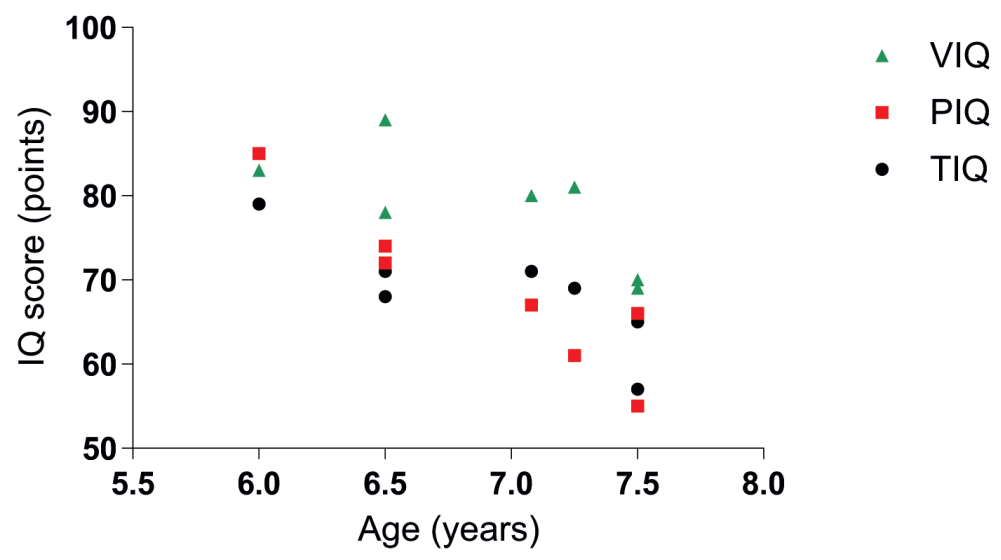

IQ scores split per IQ area in classical CLN3 disease around diagnosis. IQ score decline in classical CLN3 disease from the referral center cohort, split per IQ area. VIQ = verbal IQ; PIQ = performance IQ; TIQ = total IQ. Decline in VIQ: r-0.72 p0.0681. Decline in PIQ: $r-0.92 ; p 0.0031$. Decline in TIQ: $r-0.804 ; p 0.0293$.

\section{EARLY COGNITIVE FUNCTIONING IN DAILY LIFE}

In all classical CLN3 patients from whom information on school history was available $(n=18)$, additional educational support was provided before or at the age of 8 years explicitly noted due to learning difficulties. In contrast, all early onset Stargardt patients successfully completed regular level primary and secondary education solely requiring practical adjustments because of their visual impairment. 


\section{DISCUSSION}

In this study, we evaluated timing and severity of cognitive decline in CLN3 disease in literature-derived patients supplemented with a representative referral center cohort. Our results show that in classical CLN3 disease cognitive deficits are consistently present around the time of diagnosis. In clinical practice, school problems may be commonly attributed to rapid decrease in vision. The unaffected (childhood) neurocognitive functioning in both protracted CLN3 disease and Stargardt disease patients with similarly early and severe visual deterioration delineates that low IQ test scores and poor school performance cannot be explained by severe vision loss alone.

The present study underlines that a meta-analysis of cases obtained through a systematic literature review allows further insight into the disease course of rare disease entities, if potential drawbacks are adequately addressed (Diekman et al. 2014). To address two major drawbacks in this study - missing data and publication bias - we validated the findings of the meta-analysis in a separate cohort. The IQ scores around diagnosis in our referral center cohort analysis demonstrate that the IQ scores found in literature do not represent patients with an extraordinary early age at onset of cognitive decline. If anything, they may underestimate - rather than overestimate - cognitive function around diagnosis in classical CLN3 disease. In both the literaturederived cases and the referral center cohort, a variety of IQ tests were used and it is uncertain whether or not these tests have been adjusted to the degree of visual impairment (Adams et al. 2007). This limitation, however, also applies to the other patients tested who, despite their visual impairment, all had normal IQ scores. Furthermore, the universal presence of early learning problems in classical CLN3 patients underscores that early intellectual impairment reflects actual functional impairment in daily life.

In summary, this study demonstrates that cognitive dysfunction is universally present around diagnosis in CLN3 disease, independent of the co-occurring vision loss. In a school aged child with rapid vision loss, learning difficulties should be perceived as an important cue for CLN3 disease. 


\section{REFERENCES}

Adams HR, Kwon J, Marshall FJ, de Blieck EA, Pearce DA, Mink JW. Neuropsychological symptoms of juvenile-onset batten disease: experiences from 2 studies. J Child Neurol. 2007;22(5):621-627.

Anderson GW, Goebel HH, Simonati A. Human pathology in NCL. Biochim Biophys Acta. 2013;1832(11):1807-1826.

Diekman EF, de Koning TJ, Verhoeven-Duif NM, Rovers MM, van Hasselt PM. Survival and psychomotor development with early betaine treatment in patients with severe methylenetetrahydrofolate reductase deficiency. JAMA Neurology. 2014;71:188-194.

Haltia M. The neuronal ceroid lipofuscinosis. J Neuropathol Exp Neurol. 2003;62(1):1-13.

Kousi M, Lehesjoki AE, Mole SE. Update of the mutation spectrum and clinical correlations of over 360 mutations in eight genes that underlie the neuronal ceroid lipofuscinoses. Hum Mutat. 2012;33(1):42-63.

Kristensen K, Lou HC. Central nervous system dysfunction as early sign of neuronal ceroid lipofuscinosis (Batten's disease) Dev Med Child Neurol. 1983;25(5):588-590.

Lambertus S, van Huet RA, Bax NM, et al. Early-onset stargardt disease: phenotypic and genotypic characteristics. Ophthalmology. 2015;122(2):335-344.

Lamminranta S, Aberg LE, Autti T, et al. Neuropsychological test battery in the follow-up of patients with juvenile neuronal ceroid lipofuscinosis. J Intellect Disabil Res. 2001;45(1):817.

Lauronen L, Munroe PB, Jarvela I et al (1999). Delayed classic and protracted phenotypes of compound heterozygous juvenile neuronal ceroid lipofuscinosis. Neurology ;52(2):360-5

Munroe PB, Mitchison HM, O'Rawe AM, et al. Spectrum of mutations in the batten disease gene, CLN3. Am J Hum Genet. 1997;61(2):310-316.

Spalton DJ, Taylor DSI, Sanders MD. Juvenile Batten's disease: an ophthalmological assessment of 26 patients. Br J Ophthalmol. 1980;64:726-732. 


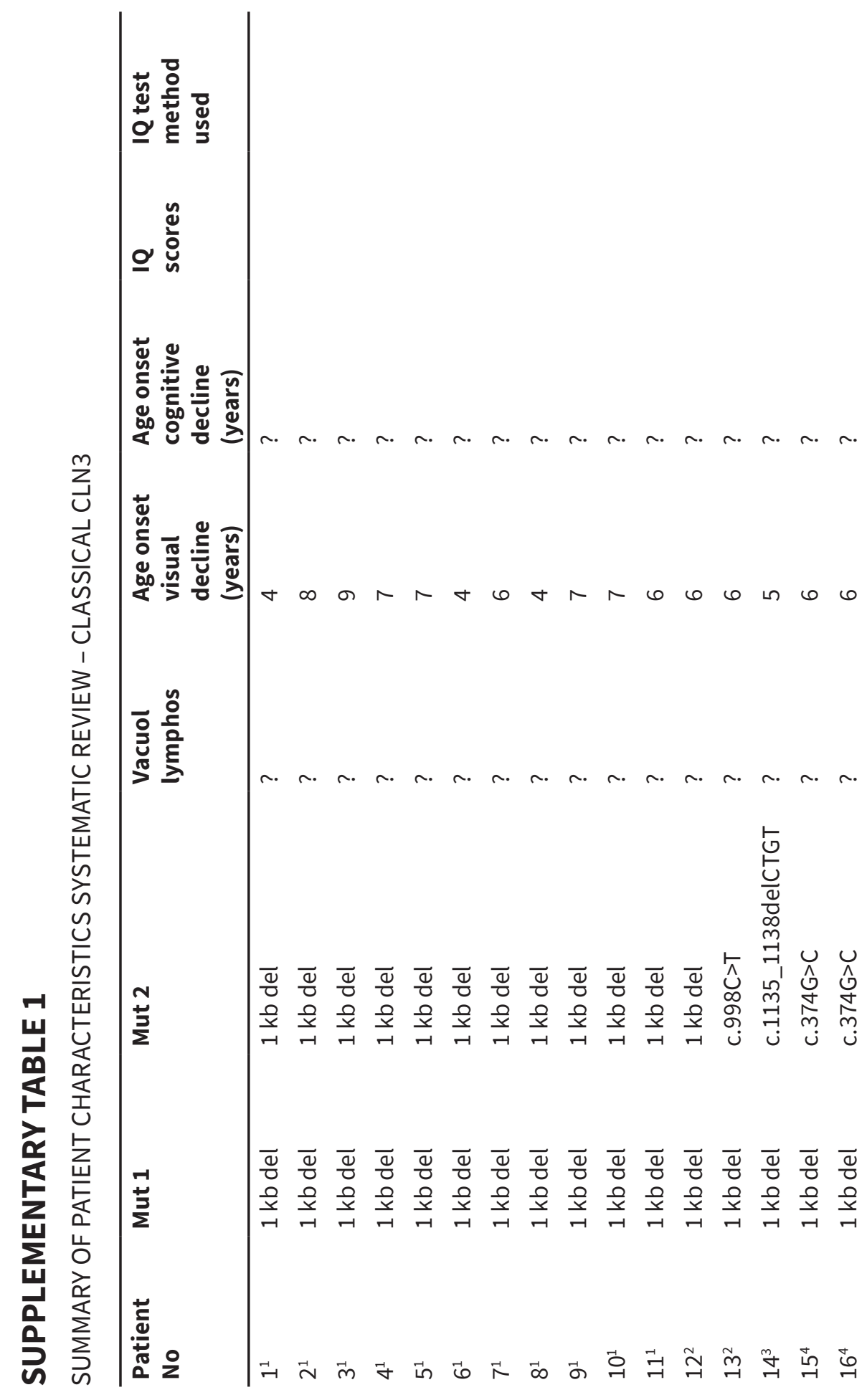




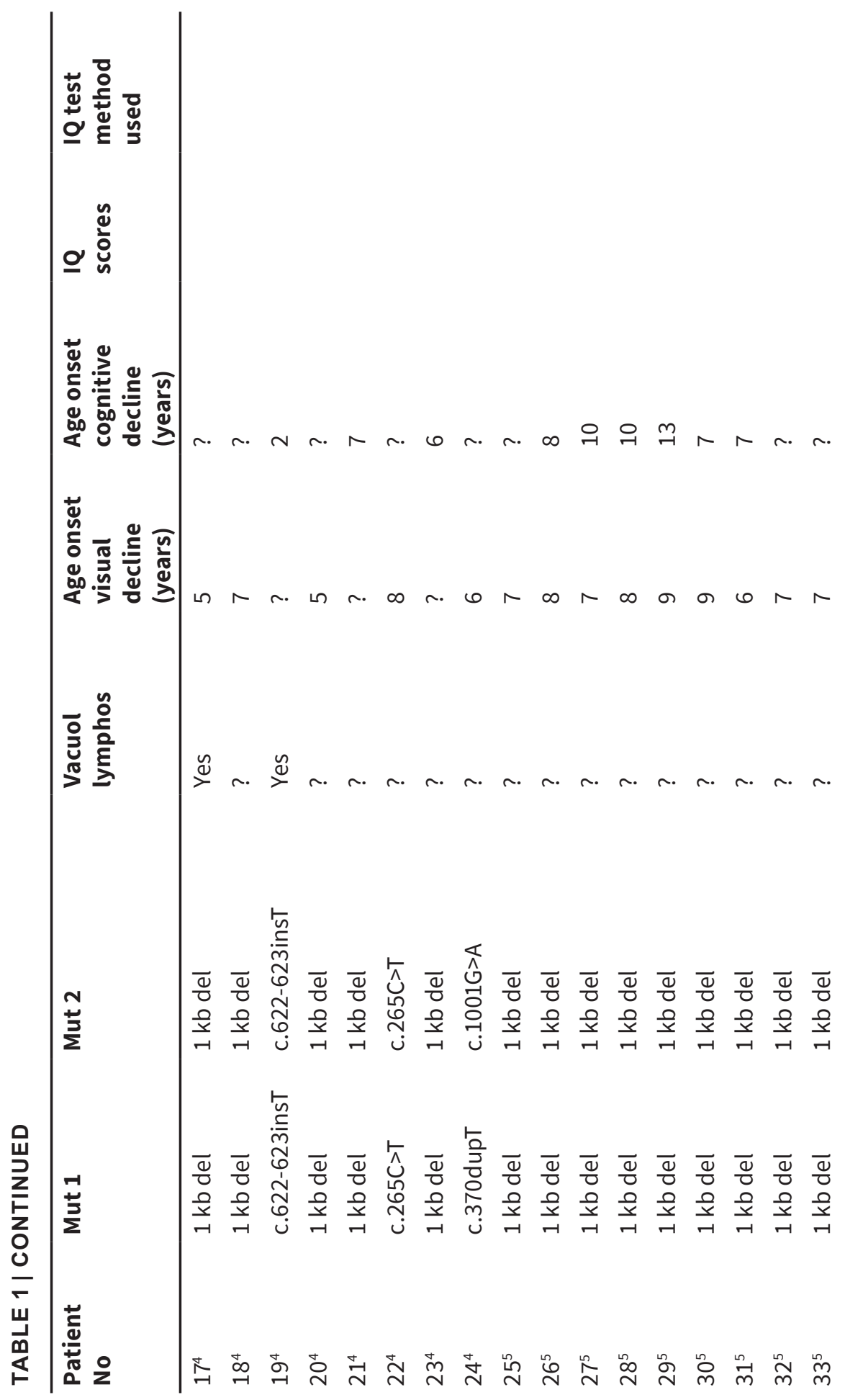




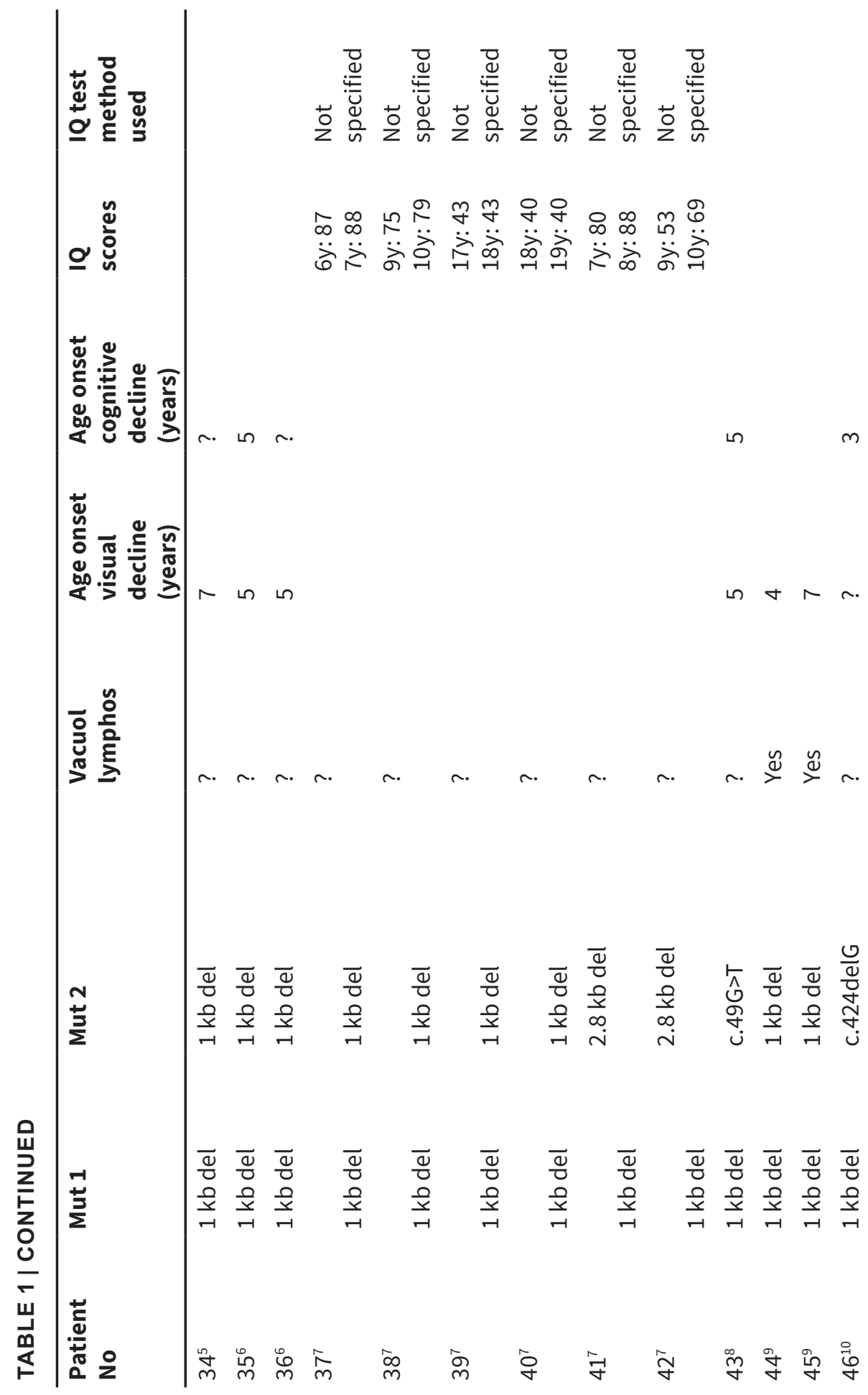




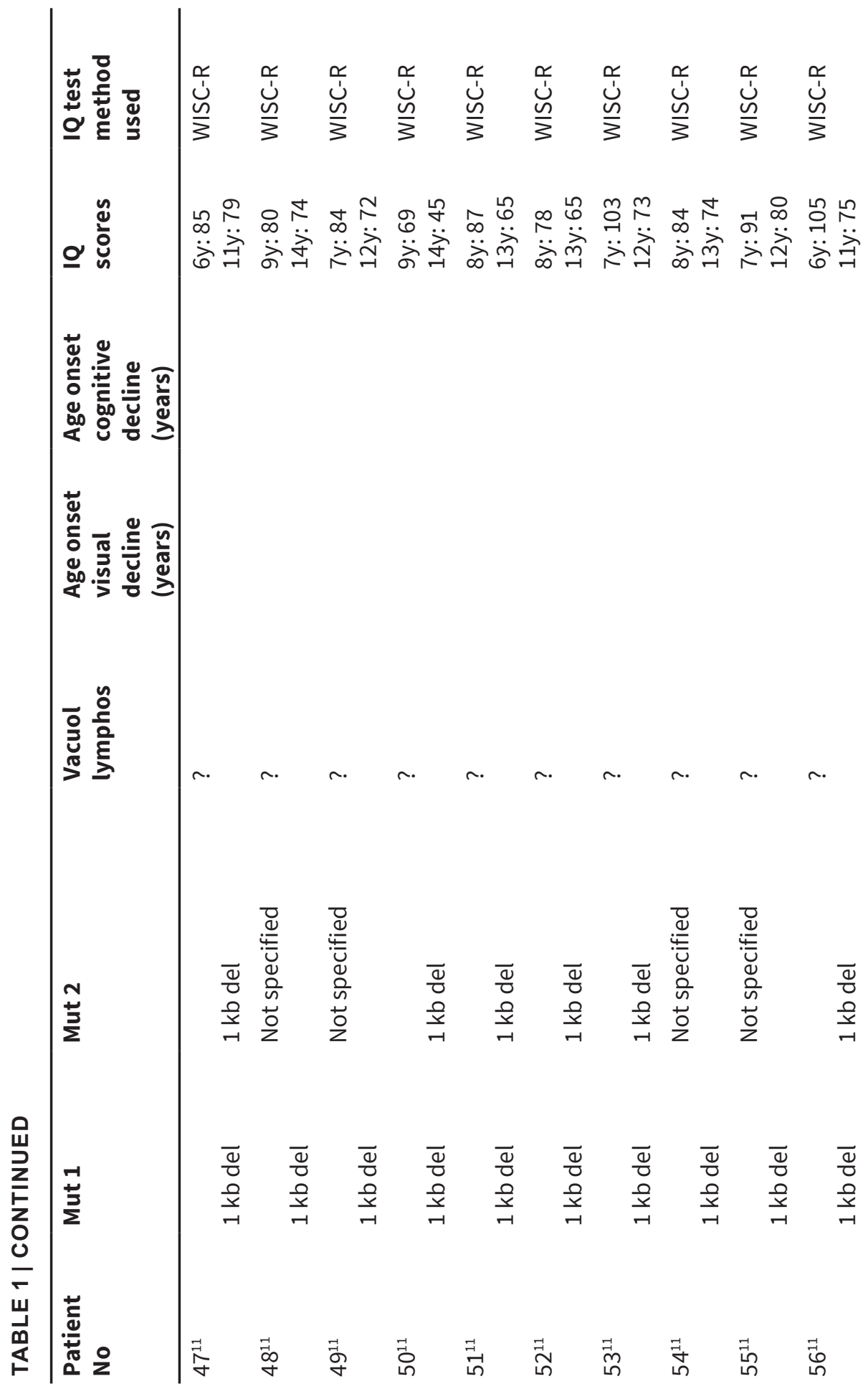




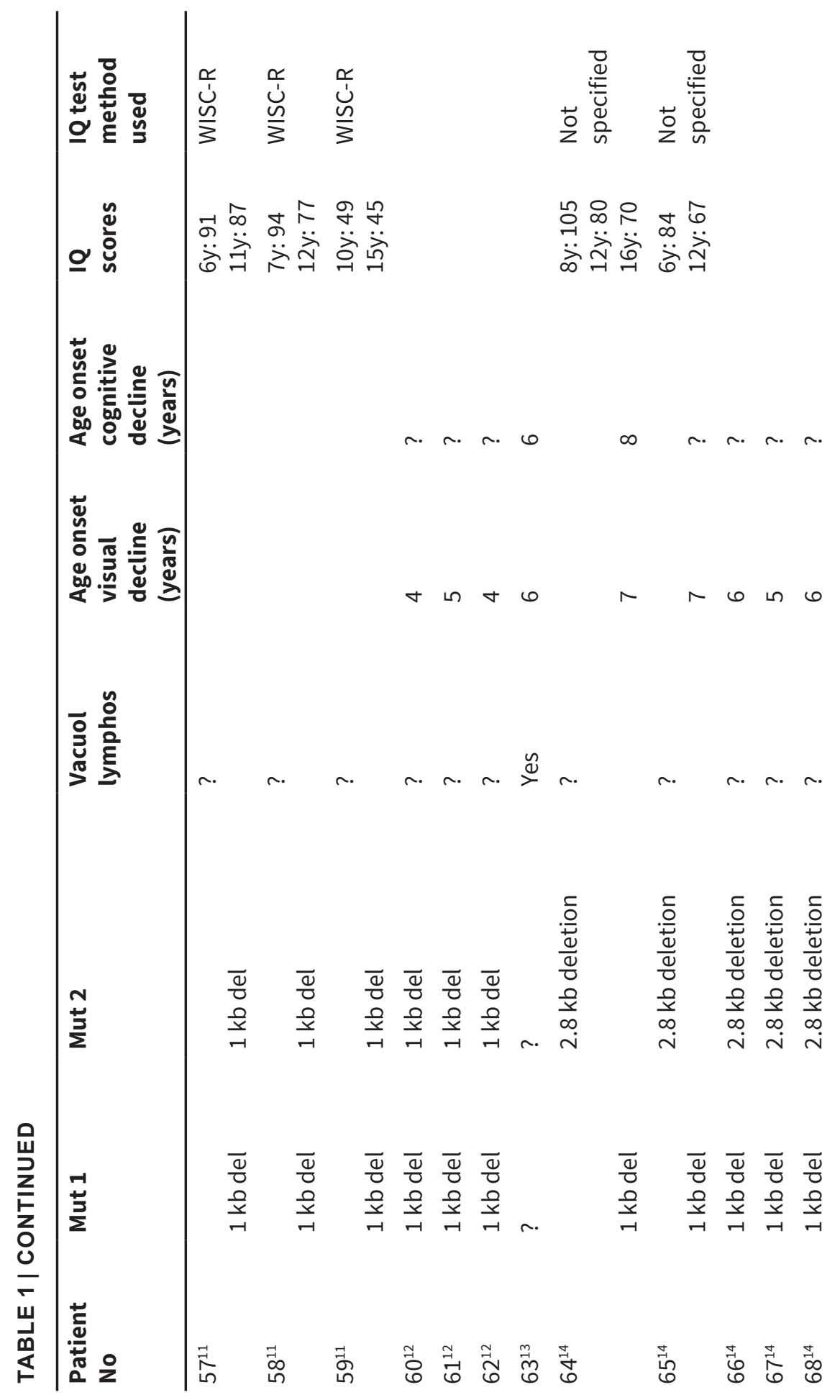




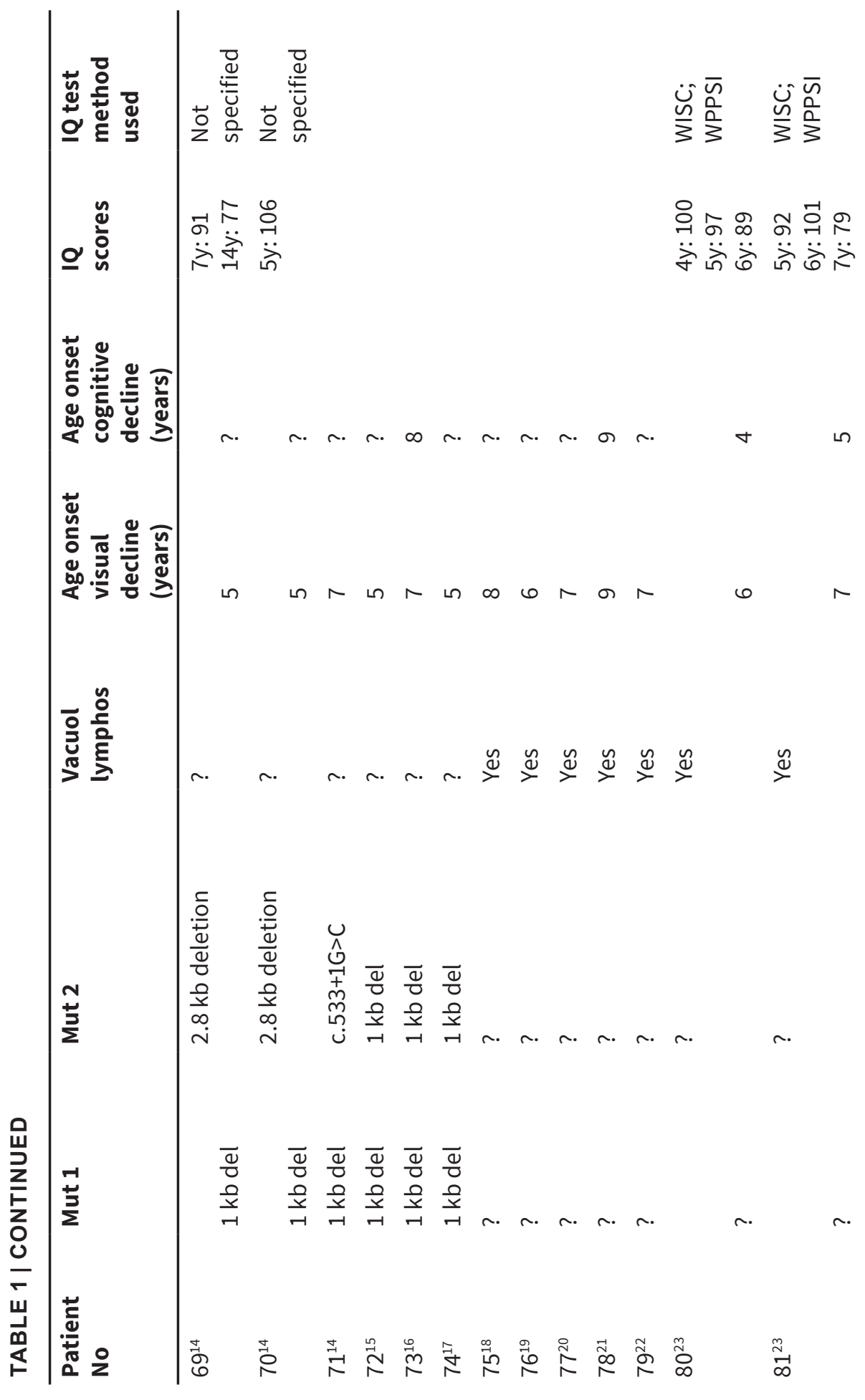




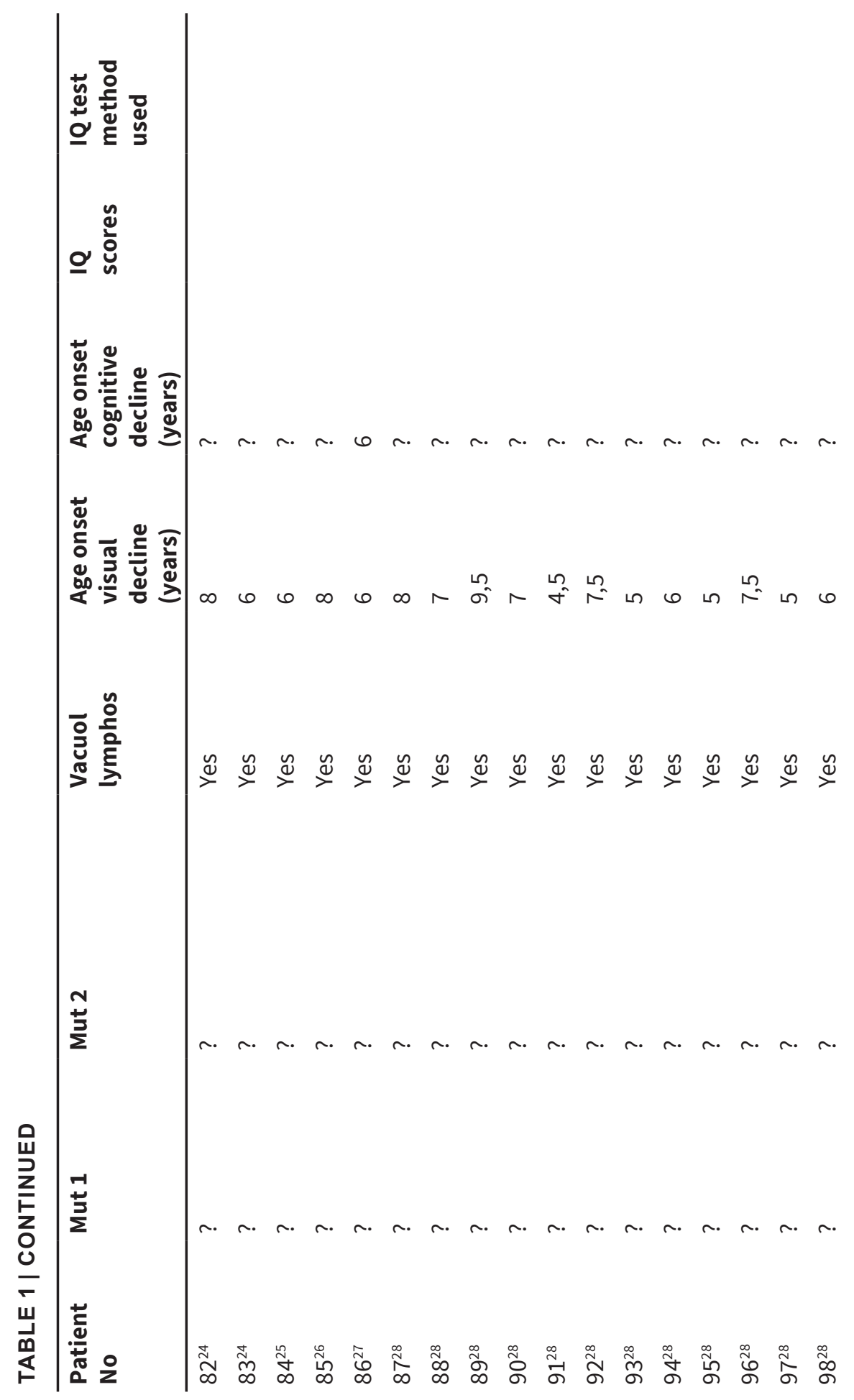




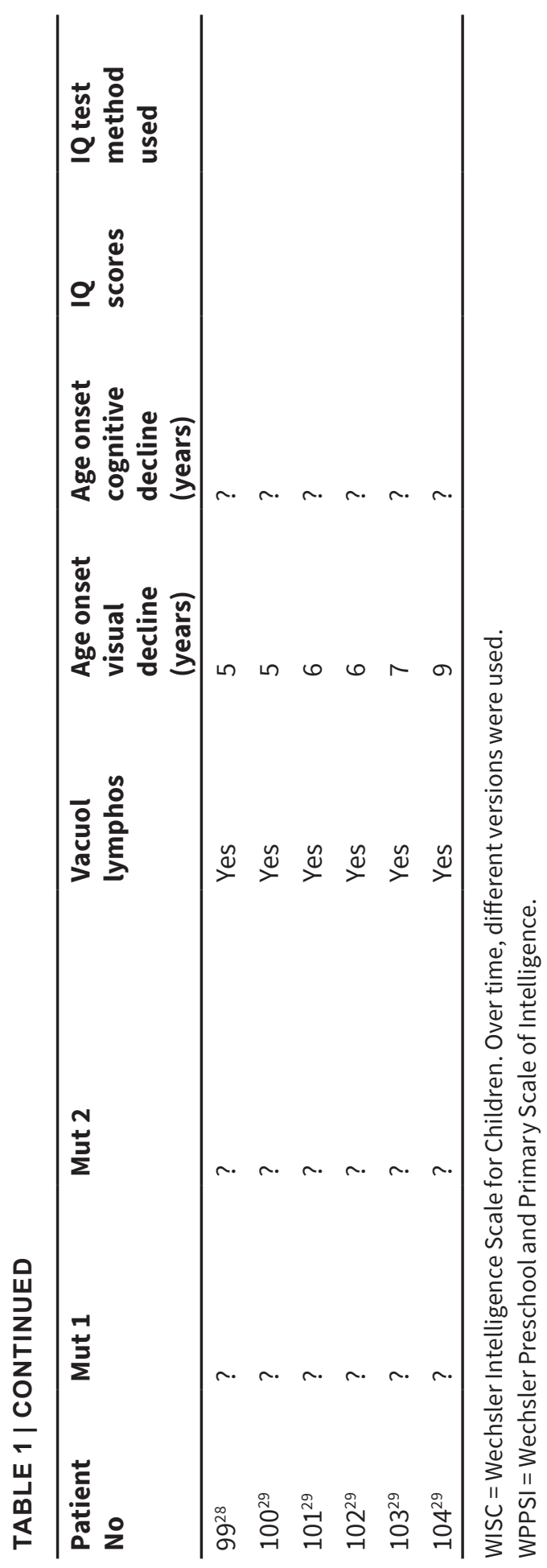




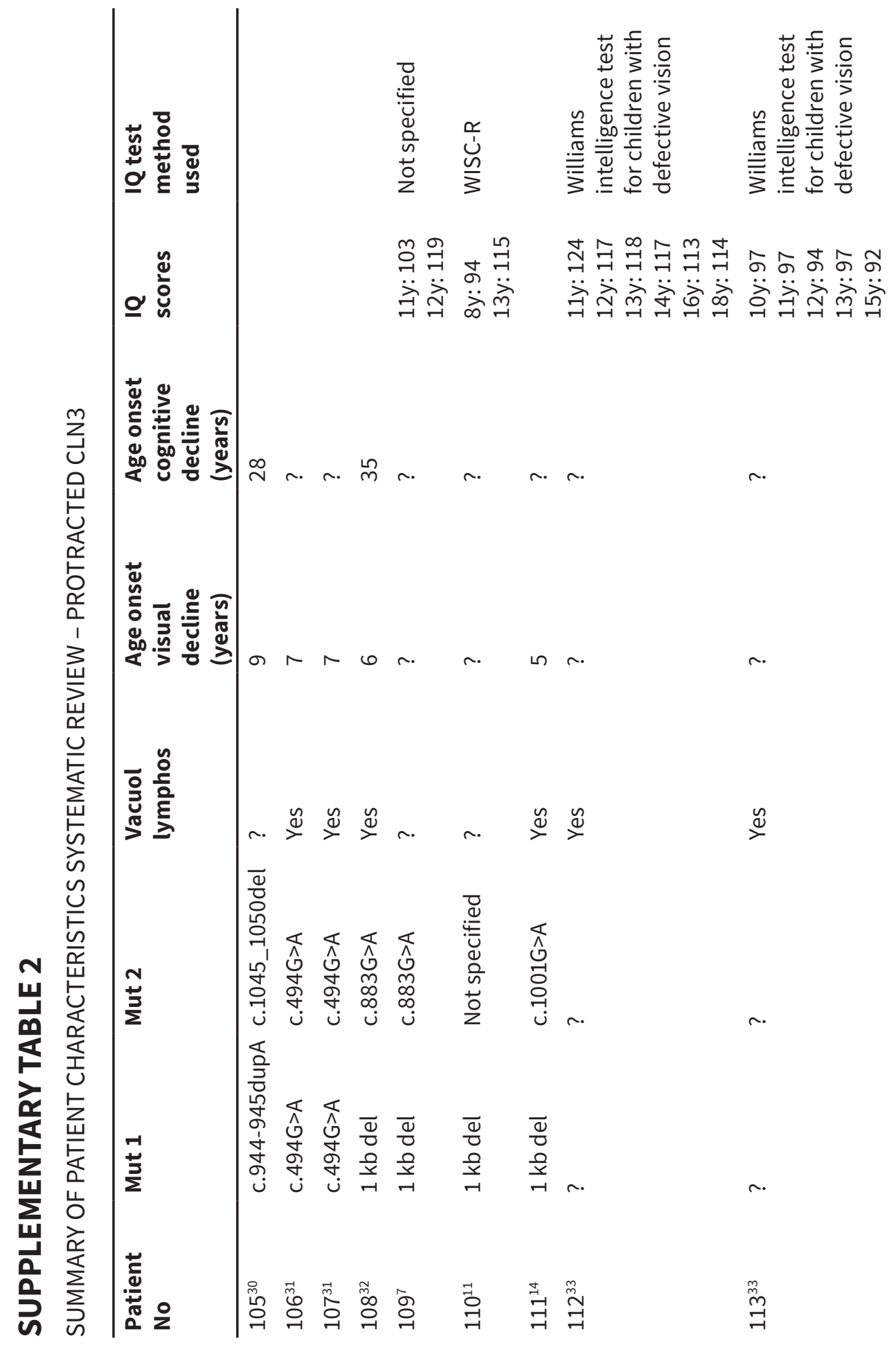




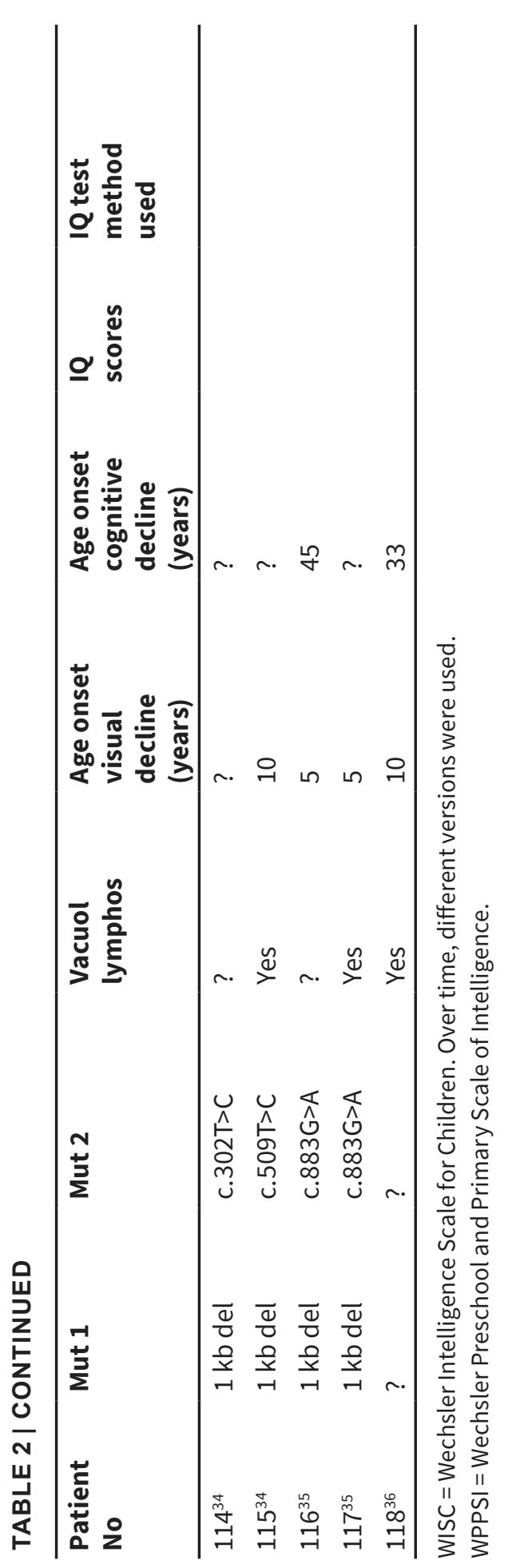




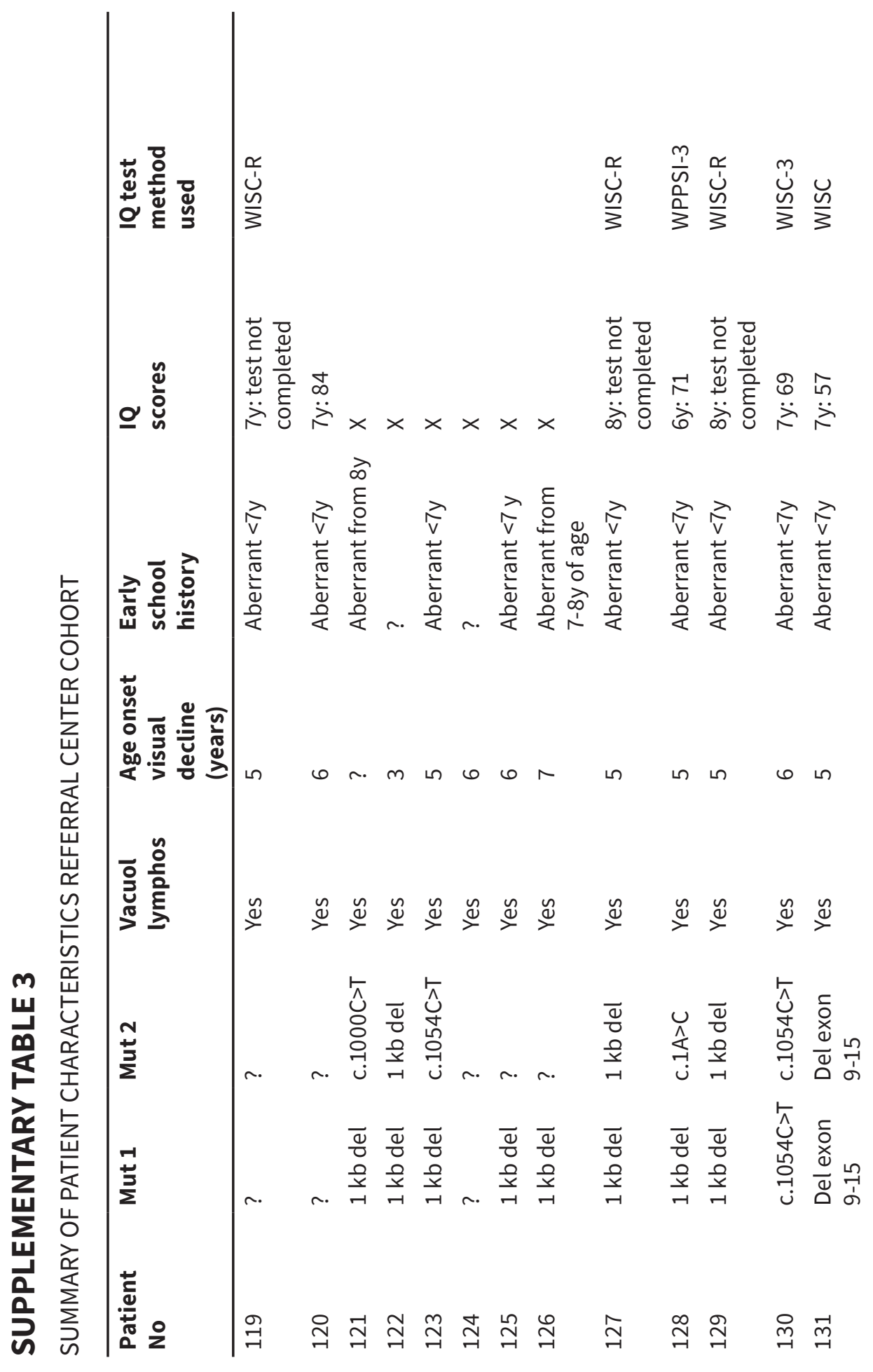




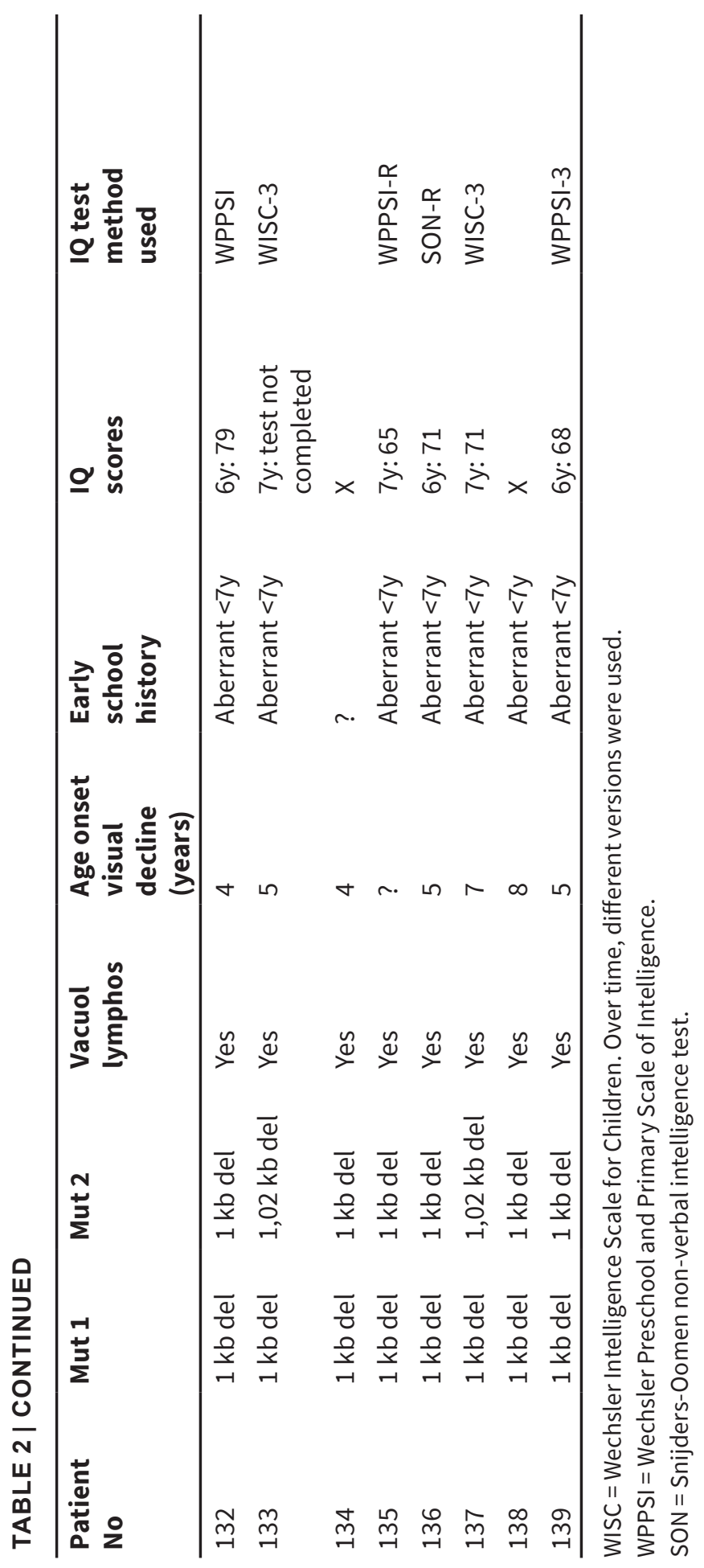




\section{SUPPLEMENTARY REFERENCES}

${ }^{1}$ Dulz S, Wagenfeld L, Nickel M et al (2016) Novel morphological macular findings in juvenile CLN3 disease. $\mathrm{Br}$ J Ophthalmol 100(6):824-8.

${ }^{2}$ Hansen MS, Hove MN, Jensen H, Larsen M (2016) OPTICAL COHERENCE TOMOGRAPHY IN JUVENILE NEURONAL CEROID LIPOFUSCINOSIS. Retin Cases Brief Rep 10(2):137-9.

${ }^{3}$ Drack AV, Miller JN, Pearce DA (2013) A novel c.1135_1138delCTGT mutation in CLN3 leads to juvenile neuronal ceroid lipofuscinosis. J Child Neurol 28(9):1112-6.

${ }^{4}$ Perez-Poyato MS, Mila Recansens M, Ferrer Abizanda I et al (2011) Juvenile neuronal ceroid lipofuscinosis: clinical course and genetic studies in Spanish patients. J Inherit Metab Dis 34:1083-1093.

${ }^{5}$ Valadares-Freitas ER, Pizarro MX, Oliveira LR, et al (2011) Juvenile neuronal ceroidlipofuscinosis: clinical and molecular investigation in a large family in Brazil. Arq Neuropsiquiatr 69:13-18.

${ }^{6}$ Elkay M, Silver K, Penn RD, Dalvi A (2009) Dystonic storm due to Batten's disease treated with pallidotomy and deep brain stimulation. Mov Disord 15;24(7):1048-53.

${ }^{7}$ Aberg L, Talling M, Harkonen T et al (2008) Intermittent prednisolone and autoantibodies to GAD65 in juvenile neuronal ceroid lipofuscinosis. Neurology 70(14):1218-20.

${ }^{8}$ Kwon JM, Rothberg PG, Leman AR, Weimer JM, Mink JW, Pearce DA (2005) Novel CLN3 mutation predicted to cause complete loss of protein function does not modify the classical JNCL phenotype. Neurosci Lett 387(2):111-4.

${ }^{9}$ Mantel I, Brantley MA Jr, Bellmann C et al (2004) Juvenile neuronal ceroid lipofuscinosis (Batten disease) CLN3 mutation (Chrom 16p11.2) with different phenotypes in a sibling pair and low intensity in vivo autofluorescence. Klin Monbl Augenheilkd 221(5):427-30.

${ }^{10}$ Vercammen L, Buyse GM, Proost JE, Van Hove JL (2003) Neuroleptic malignant syndrome in juvenile neuronal ceroid lipofuscinosis associated with low-dose risperidone therapy. $J$ Inherit Metab Dis 26(6):611-2.

${ }^{11}$ Lamminranta S, Aberg LE, Autti T et al (2001) Neuropsychological test battery in the followup of patients with juvenile neuronal ceroid lipofuscinosis. J Intellect Disabil Res 45(Pt 1):8-17.

${ }^{12}$ Bohra LI, Weizer JS, Lee AG, Lewis RA (2000) Vision loss as the presenting sign in juvenile neuronal ceroid lipofuscinosis. J Neuroophthalmol 20(2):111-5.

${ }^{13}$ Villanova M, Ceuterick C, Dotti MT et al (1999) Detection of beta-A4 amyloid and its precursor protein in the muscle of a patient with juvenile neuronal ceroid lipofuscinosis (SpielmeyerVogt-Sjogren). Acta Neuropathol 98(1):78-84.

${ }^{14}$ Lauronen L, Munroe PB, Jarvela I et al (1999) Delayed classic and protracted phenotypes of compound heterozygous juvenile neuronal ceroid lipofuscinosis. Neurology 15;52(2):3605.

${ }^{15}$ Inan C, Wong D, Wisniewski KE, Rose AL, Musarella MA (1998) First African-American child with juvenile neuronal ceroid lipofuscinosis. Am J Med Genet 79(5):335-6.

${ }^{16}$ Weleber RG (1998) The dystrophic retina in multisystem disorders: the electroretinogram in neuronal ceroid lipofuscinoses. Eye (Lond) 12(Pt 3b):580-90.

${ }^{17}$ Aberg L, Jarvela I, Rapola J et al (1998) Atypical juvenile neuronal ceroid lipofuscinosis with granular osmiophilic deposit-like inclusions in the autonomic nerve cells of the gut wall. Acta Neuropathol 95(3):306-12.

${ }^{18}$ Marshman WE, Lee JP, Jones B, Schalit G, Holder GE (1998) Duane's retraction syndrome and juvenile Batten's disease: a new association? Aust N Z J Ophthalmol 26(3):251-4. 
${ }^{19}$ Crow YJ, Tolmie JL, Howatson AG, Patrick WJ, Stephenson JB (1997). Batten disease in the west of Scotland 1974-1995 including five cases of the juvenile form with granular osmiophilic deposits. Neuropediatrics 28(3):140-4.

${ }^{20}$ Lake BD, Steward CG, Oakhill A, Wilson J, Perham TG (1997). Bone marrow transplantation in late infantile Batten disease and juvenile Batten disease. Neuropediatrics 28(1):80-1.

${ }^{21}$ Taschner PE, de Vos N, Thompson AD et al (1995) Chromosome 16 microdeletion in a patient with juvenile neuronal ceroid lipofuscinosis (Batten disease). Am J Hum Genet 56(3):663-8.

22 Horiguchi M, Miyake Y (1992) Batten disease--deteriorating course of ocular findings. Jpn J Ophthalmol 36(1):91-6.

${ }^{23}$ Kristensen K, Lou HC (1983) Central nervous system dysfunction as early sign of neuronal ceroid lipofuscinosis. Dev Med Child Neurol 25(5):588-90.

${ }^{24}$ Seeliger M, Rüther K, Apfelstedt-Sylla E, Schlote W, Wohlrab M, Zrenner E (1997) Juvenile neuronal ceroid lipofuscinosis (Batten-Mayou) disease. Ophthalmologic diagnosis and findings. Ophthalmologe 94(8):557-62.

${ }^{25}$ Henry JG, Stevens SM (1982) Neuronal ceroid lipofuscinosis in the amaurotic retardate: electron microscopic confirmation. Aust J Ophthalmol 10(3):161-6.

${ }^{26}$ Johnson J (1961) Juvenile amaurotic idiocy: a case report. J Ment Sci 107:931-5.

${ }^{27}$ Piatella L, Cardinali C, Zamponi N, Papa O (1991) Spielmeyer-Vogt disease: clinical and neurophysiological aspects. Child's Nerv Syst 7:226-230.

${ }^{28}$ Jensen GE, Clausen J, Melchior JC, Konat G (1977) Clinical, social and biochemical studies on Batten's syndrome, alias Spielmeyer-Vogt or Stengel's syndrome. Eur Neurol 15(4):203-11.

${ }^{29}$ Nardocci N, Verga ML, Binelli S, Zorzi G, Angelini L, Bugiani O (1995) Neuronal ceroidlipofuscinosis: a clinical and morphological study of 19 patients. Am J Med Genet 5;57(2):137-41.

${ }^{30}$ Licchetta L, Bisulli F, Fietz M t al (2015) A novel mutation of $\mathrm{Cln} 3$ associated with delayedclassic juvenile ceroid lipofuscinois and autophagic vacuolar myopathy. Eur J Med Genet 58(10):540-544.

${ }^{31}$ Cortese A, Tucci A, Piccolo G et al (2014) Novel CLN3 mutation causing autophagic vacuolar mypopathy. Neurology 10;82(23):2072-6.

${ }^{32}$ Aberg L, Lauronen L, Hämäläinen J, Mole SE, Autti T (2009) A 30-year follow-up of a neuronal ceroid lipofuscinosis patient with mutations in CLN3 and protracted disease course. Pediatr Neurol 40(2):134-7.

${ }^{33}$ Bennett MJ, Gayton AR, Rittey CD, Hosking GP (1994) Juvenile neuronal ceroid-lipofuscinosis: developmental progress after supplementation with polyunsaturated fatty acids. Dev Med Child Neurol 36(7):630-8.

${ }^{34}$ Munroe PB, Mitchison HM, O'Rawe AM et al (1997) Spectrum of mutations in the Batten disease gene, CLN3. Am J Hum Genet 61(2):310-6.

${ }^{35}$ Wisniewski KE, Zhong N, Kaczmarski W et al (1998) Compound heterozygous genotype is associated with protracted juvenile neuronal ceroid lipofuscinosis. Ann Neurol 43(1):10610.

${ }^{36}$ Goebel HH, Pilz H, Gullota F (1976) The protracted form of juvenile neuronal ceroidlipofuscinosis. Acta Neuropathol 36(4):393-6. 
|2 



\section{CHAPTER MOTOR FUNCTION IMPAIRMENT IS AN EARLY SIGN OF CLN3 DISEASE}

Willemijn F.E. Kuper, Claudia van Alfen, Linda van Eck, Barbara C.H. Huijgen, Edward E.S. Nieuwenhuis, Marco van Brussel, Peter M. van Hasselt Neurology. 2019 Jul 16;93(3):e293-e297

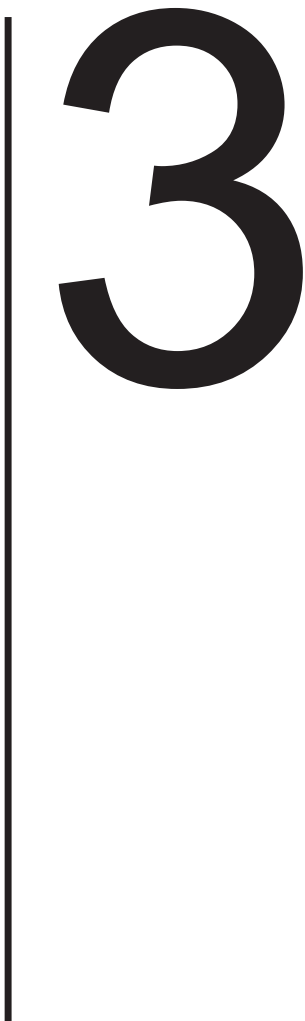




\section{ABSTRACT}

Objective: To delineate timing of motor decline in CLN3 disease.

Methods: Motor function, assessed by the 6-Minute Walk Test (6MWT), was evaluated repeatedly in 15 patients with CLN3 disease, resulting in 65 test results and during one occasion in 2 control cohorts. One control cohort $(n=14)$ had isolated visual impairment; a second cohort $(n=12)$ exhibited visual impairment in combination with neurologic impairments. Based on 6MWT reference values in healthy sighted children, $z$ scores of 6MWT results in patients with CLN3 disease and control cohort individuals were calculated. 6MWT results were correlated with age-including multilevel modeling analysis allowing assessment of imbalanced repeated measurements-and with Unified Batten Disease Rating Scale (UBDRS) scores.

Results: In CLN3 disease, 6MWT scores were already impaired from first testing near diagnosis (mean z scores of -3.6 and -4.7 at 7 and 8 years of age, respectively). Afterwards, 6MWT scores continuously declined with age $(r=-0.64, p<0.0001)$ and with increasing UBDRS scores $(r=-0.60, p=0.0001)$, confirming correlation with disease progression. The decrease was more pronounced at a later age, as shown by the nonlinear multilevel model for 6MWT results in CLN3 disease ( $y=409.18-[0.52 \times$ age2]). In contrast, an upward trend of 6MWT scores with age was observed in the control cohort with isolated visual impairment $(r=0.56 ; p=0.04)$ similar to healthy, sighted children. The control cohort with additional neurologic impairments displayed a slightly decreased 6MWT walking distance independent of age.

Conclusions: The 6MWT unveils early onset of motor decline in CLN3 disease. 


\section{INTRODUCTION}

CLN3 disease (OMIM \#204200), formerly known as juvenile neuronal ceroid lipofuscinosis or Batten disease, is a neurodegenerative lysosomal storage disorder with childhood onset. Currently, there are no curative or diseasemodifying treatments for CLN3 disease. As a consequence, patients usually die in their second or third decade of life. ${ }^{1}$ The most striking early clinical feature is vision loss. This was long assumed to precede progressive neurologic deterioration by a few years, implicating a therapeutic window for any future brain-targeting treatments. ${ }^{2-4}$ Recently, however, onset of vision loss was found to coincide with onset of cognitive decline, rather implying early brain involvement. ${ }^{5}$ This led us to hypothesize that motor function decline might also have its onset early in the disease course.

The 6-Minute Walk Test (6MWT) is a clinical evaluation tool to obtain an overall impression of motor functioning. The 6MWT is commonly used to assess disease progression in various clinical populations; however, to our knowledge, it has not yet been described in visually impaired individuals, including CLN3 disease. ${ }^{6,7}$ We used the 6MWT to delineate timing of motor function decline in CLN3 disease.

\section{METHODS}

\section{STUDY POPULATION}

From 2012 to 2017, 15 patients with a genetically confirmed diagnosis of CLN3 disease (9 patients homozygous for the common $1 \mathrm{~kb}$ deletion, 4 patients compound heterozygous for the $1 \mathrm{~kb}$ deletion and a different deletion or missense mutation, 1 patient homozygous for a different deletion, and 1 patient homozygous for a nonsense mutation) performed a total of 65 6MWT tests (mean of 3.8 6WMT performances per patient with CLN3 disease). All patients with CLN3 disease had a severe visual impairment: around diagnosis at a median age of 7 years (range 5-12 years), visual acuity was without exception below 0.3 -considered low vision according to WHO guidelines ${ }^{8}-$ deteriorating to blindness in a few years (data not shown). To determine the effect of visual impairment on motor functioning, 2 control cohorts totaling 26 children from the affiliated school for visually impaired children also performed the 6MWT during one occasion using the same protocol. Fourteen of these 26 children had an isolated visual impairment, thus without additional 
neurologic impairments, and the remaining 12 exhibited visual impairment in combination with neurologic impairments. A complete overview of the control cohorts is provided in the table. Of note, visual acuity can be reported in fractions, a logarithmic scale, or decimals, often dependent on the assessment method used. To make the visual acuities comparable, we chose to recalculate all visual acuities in decimals (e.g., 20/200 equals 0.1 ) if not already reported so. Reference values for 6MWT distances in healthy sighted children were retrieved from Ulrich et al., ${ }^{9}$ based on the decision tree described by Mylius et al. ${ }^{7}$

\section{MWT PROTOCOL}

The 6MWT was performed biannually during our multidisciplinary CLN3 disease follow-up meetings under the guidance of the same experienced physical therapist (L.v.E.). We applied a slightly adapted protocol derived from the standard American Thoracic Society guidelines. ${ }^{6}$ The assessments were performed indoors on a smooth, uncluttered surface in the physical rehabilitation accommodations at the Bartiméus center. Due to limitations of space, lanes of 10 meters rather than the standard 30 meters were marked on which the patients and the control group individuals had to walk back and forth. However, as both the patients and the control group individuals had to walk on these shorter lanes, any speed loss and thus 6MWT results impairment should not have affected the comparative analysis. Due to the visual impairments present in both the patients and control cohorts, they were allowed to-dependent on their preferences and abilities-walk independently, with a walking cane, or with physical assistance of the physical therapist. In all cases, the individual that was tested determined the tempo.

\section{DATA ANALYSIS}

For data analysis, we retrieved distance walked in meters and age at testing. Based on the 6MWTreference values in healthy sighted children, we calculated $z$ scores of the 6MWT results in patients with CLN3 disease and control cohort individuals. 6MWT results were correlated with age. To account for repeated measurements with missing data in the patients with CLN3 disease, multilevel analysis was used. Multilevel analysis, modeling all measurements (level 1) within patients (level 2) with CLN3 disease, was performed with 6MWT scores as the outcome measurement. Age was added to the multilevel model, to validate a possible relation between 6MWT scores and age. ${ }^{10}$ Both age and quadratic age were entered in the model to find the best model fit. This indicates if the best model fit is a linear or a quadratic curve relationship. The 
Unified Batten Disease Rating Scale (UBDRS) assesses multiple domains of disease in detail-including a physical assessment, seizure assessment, and capability assessment including school performance-and provides a clinical global impression score of these disease domains. ${ }^{11}$ If during the same visit both the 6MWT and the UBDRS were applied, we correlated 6MWT scores with the sum of the UBDRS clinical global impression scores.

Statistical analyses were performed using Graphpad Prism version 7.02 and MLwiN version 3.01 software. For correlation analyses, Pearson R coefficient was used. $p$ Values $<0.05$ were considered statistically significant.

STANDARD PROTOCOL APPROVALS, REGISTRATIONS, AND PATIENT CONSENTS

The study was approved by the Medical Ethical Review Committee of the University Medical Center Utrecht (17-269/C).

DATA AVAILABILITY

Anonymized data will be shared by request from any qualified investigator.

\section{RESULTS}

In patients with CLN3 disease, 6MWT scores were already impaired around diagnosis (mean $z$ score of $-3.6[n=3]$ at 7 years of age; mean $z$ score of -4.7 at 8 years of age $[n=5]$ ) (Figure 1 6MWT scores in CLN3 disease and figure 2 corresponding $z$ scores). Afterwards, a continuous decline with increasing age was seen $(r=-0.64, p<0.0001)$ (Figure 1). Correspondingly, 6MWT scores decreased with increasing UBDRS scores, confirming correlation with disease progression ( $n=37$ corresponding 6MWT and UBDRS measurements, $r=-0.60$, $p=0.0001)$ (data not shown). 
FIGURE 1 | SIX-MINUTE WALK TEST (6MWT) PERFORMANCE IN PATIENTS WITH CLN3 DISEASE

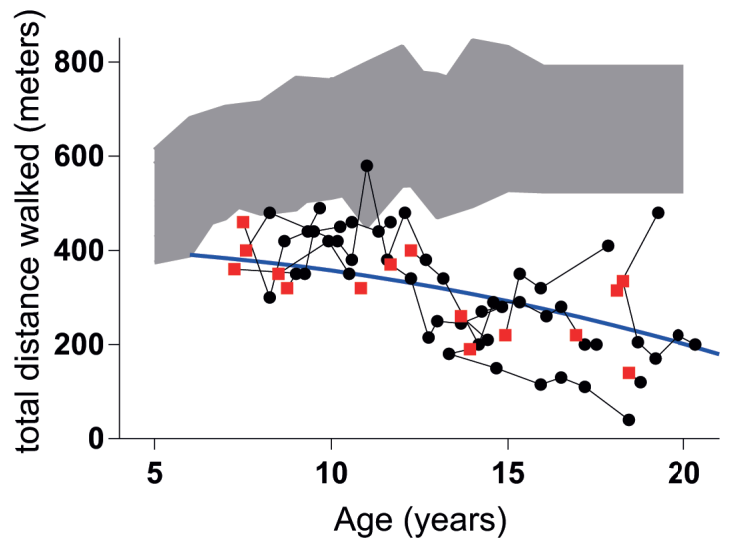

6MWT scores over time in patients with CLN3 disease ( $n=65$ test scores from $n=15$ patients, $r=-0.64, p<0.0001$ ). Median age at first 6MWT was 13 years (range $7-18$ years); median age at last 6MWT was 14 years (range 9-20 years). First measurement per patient is shown in red squares. Subsequent measurements are shown in black circles. Official reference values in healthy sighted children are provided in gray (mean $\pm 2 S D$ ). The nonlinear multilevel model is provided in blue $\left(y=409.18-\left[0.52 \times\right.\right.$ age $\left.\left.^{2}\right]\right)$.

Although 6MWT results already decreased in the early stages of the disease $(r=-0.46$ up to and including 13 years of age, $p=0.005)$, the decrease in motor function was as expected more pronounced at a later age, indicating that a linear regression model may not provide the most accurate model to use the 6MWT as a follow-up marker. To account for this nonlinear decrease with age, we therefore used a nonlinear multilevel model that provided us with the following equation for 6MWT results decline in CLN3 disease: $y=409.18$ - (0.52 $\times$ age 2$)$. To assess whether the decreased 6MWT walking distance could be attributed to the visual impairment, we additionally performed the 6MWT in the first control cohort with a severe but isolated visual impairment (table). In contrast with the test results in patients with CLN3 disease, 6MWT scores in children with an isolated visual impairment showed an upward trend with age $(r=0.56 ; p=0.04)$ similar to healthy sighted children (mean $z$ score -0.38 ) (figure 3). There was no correlation between the degree of visual impairment and 6MWTz score (data not shown), indicating that the degree of visual impairment did not directly affect $6 \mathrm{MWT}$ results. To assess the effect 
of neurologic impairment on 6MWT walking distance, we also performed the 6MWT in a control cohort displaying mild visual impairment in addition to non-progressive neurologic impairments (table). Within this control cohort, 6MWT scores were slightly impaired (mean z score -1.26 ) without an upward or downward trend with age $(r=-0.29 ; p=0.36)$ (Figure 3).

FIGURE 2 | SIX-MINUTE WALK TEST (6MWT) Z SCORES IN CLN3 DISEASE

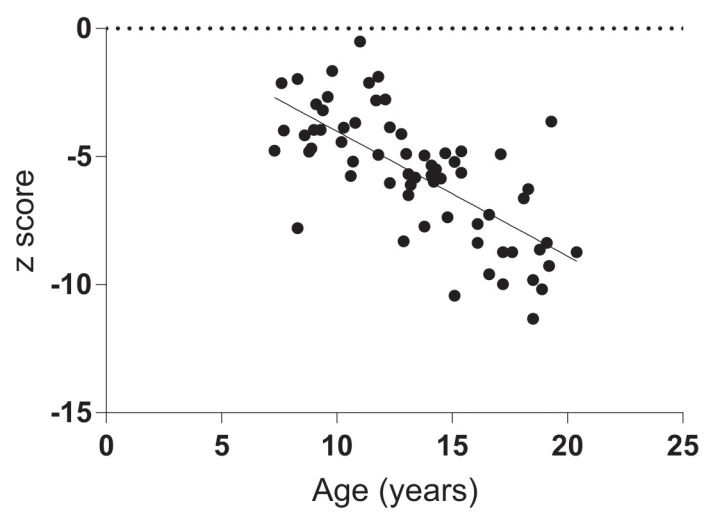

z-scores derived from 6MWT scores in patients with CLN3 disease compared to the reference values in healthy sighted children $(r=-0.70 ; p<0.0001)$.

FIGURE 3 | SIX-MINUTE WALK TEST (6MWT) PERFORMANCE IN (ISOLATED) VISUALLY IMPAIRED CHILDREN

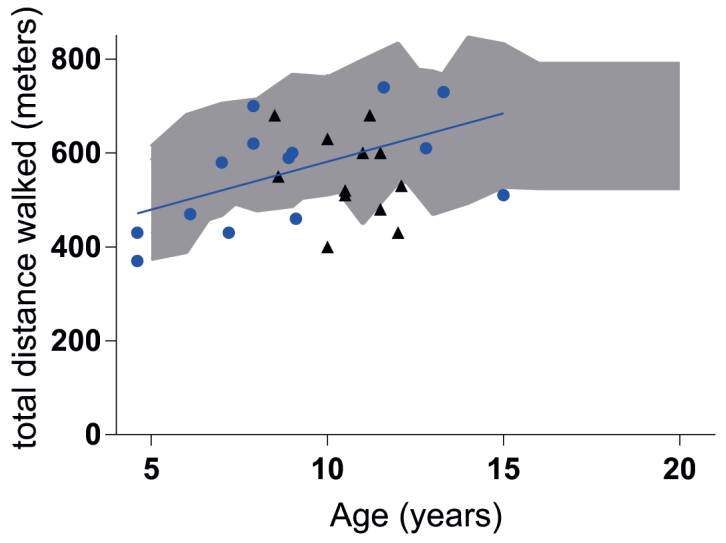


6MWT scores over time in children with a visual impairment (see table for individual diagnoses and test results). Test results from children with an isolated visual impairment are shown in blue circles $(n=14, r=0.56 ; p=0.04)$. Test results from children with visual and neurologic impairments are shown in black triangles $(n=12, r=-0.29 ; p=0.36)$. Official reference values in healthy sighted children are provided in gray (mean $\pm 2 S D$ ).

\section{DISCUSSION}

We provide evidence that in CLN3 disease, motor function is already impaired at the time of diagnosis.

The decreased 6MWT distance walked by patients with CLN3 disease cannot be explained by their visual impairment. The control cohort of individuals with a severe but isolated visual impairment performed the 6MWT similarly well compared to their sighted peers. Conversely, the group with mild impairments of visual acuity but accompanying neurologic impairments displayed a decreased 6MWT walking distance. Of note, they still outperformed patients with CLN3 disease-even those early in the disease course-underlining the severity of motor impairment in CLN3 disease as detected by the 6MWT.

As the 6MWT assesses functional exercise capacity deriving from multiple aspects involved in disease, ${ }^{4}$ it is not possible to retrieve a single cause for impaired 6WMT scores. As CLN3 disease is known to cause a parkinsonismlike motor dysfunction ${ }^{12}$-a combination of reduced walking speed, smaller steps, and start-stop problems-it is tempting to speculate that the gradual decrease in ambulation from early in the disease course onwards reflects subclinical parkinsonism. Attention span may also be a relevant factor, particularly early in the disease. ${ }^{13,14}$ Indeed, the physical therapist who guided all 6MWTs mentioned that the patients with CLN3 disease, already from an early age, seemed easily distracted and hence seemed to have difficulties keeping themselves focused on the task. Of note, while neuronal loss is the main cause of its deteriorating course, CLN3 disease is a systemic disease. ${ }^{15}$ Signs of accumulation in skeletal muscle have been described, but without any clear clinical implications. ${ }^{16}$ In contrast, cardiac muscle involvement has been described late in the disease course. ${ }^{17}$ Therefore, we cannot exclude a role for cardiac or even skeletal muscle impairment in the decreased functional exercise capacity earlier in the disease course. 
We show that motor decline has its onset early in the disease course of CLN3 disease, which is quantifiable using the 6MWT and cannot be explained by decreased visual acuity. We encourage using the 6MWT for CLN3 disease progression assessment in clinical follow-up and future therapeutic studies.

TABLE | CONTROL GROUP CHARACTERISTICS

\begin{tabular}{|c|c|c|c|c|c|c|}
\hline $\begin{array}{l}\text { No. of } \\
\text { patients } \\
\text { (total } \\
\mathrm{n}=26 \text { ) }\end{array}$ & Visual diagnosis & $\begin{array}{l}\text { Latest } \\
\text { known } \\
\text { visual } \\
\text { acuity in } \\
\text { decimals }\end{array}$ & $\begin{array}{l}\text { Neurologic } \\
\text { impairment }\end{array}$ & $\begin{array}{l}\text { Age at } \\
\text { testing } \\
\text { (years) }\end{array}$ & $\begin{array}{l}6 \mathrm{MWT} \\
\text { results } \\
\text { in } \\
\text { meters }\end{array}$ & $\begin{array}{l}\text { 6MWT } \\
\text { z-score }\end{array}$ \\
\hline
\end{tabular}

Isolated visual impairment

(14)

\begin{tabular}{lllllll}
\hline 1 & $\begin{array}{l}\text { Leber congenital } \\
\text { amaurosis }\end{array}$ & $\begin{array}{l}\text { Legally } \\
\text { blind }\end{array}$ & - & $15 y$ & 510 & -2.5 \\
3 & Stargardt & 0.1 & - & & & \\
& disease/cone-rod & 0.25 & & 7.0 & 580 & -0.45 \\
& $\begin{array}{l}\text { dystrophy/retinal } \\
\text { dystrophy }\end{array}$ & 0.1 & & 7.9 & 700 & 1.9 \\
& & & 12.8 & 610 & -1.0 \\
2 & (Congenital) & 0.2 & - & & & \\
& nystagmus & 0.25 & & 7.2 & 430 & -3.4 \\
3 & Achromatopsia & 0.1 & - & 9.0 & 600 & -0.11 \\
& & 0.16 & & 6.1 & 470 & -1.4 \\
& & 0.1 & & 9.1 & 460 & -2.8 \\
2 & Oculo(cutaneous) & 0.1 & - & 13.3 & 730 & 1.9 \\
& albinism & 0.2 & & 4.6 & 430 & -1.1 \\
1 & Congenital & 0.08 & - & 7.9 & 620 & 0.33 \\
& cataract & & & 4.6 & 370 & -2.1 \\
1 & Retinoblastoma & 0.25 & - & & & \\
1 & Stickler & 0.12 & - & 11.6 & 740 & 1.3 \\
& syndrome & & & 8.9 & 590 & -0.10 \\
& & & & &
\end{tabular}


TABLE | CONTINUED

\begin{tabular}{|c|c|c|c|c|c|c|}
\hline $\begin{array}{l}\text { No. of } \\
\text { patients } \\
\text { (total } \\
n=26 \text { ) }\end{array}$ & Visual diagnosis & $\begin{array}{l}\text { Latest } \\
\text { known } \\
\text { visual } \\
\text { acuity in } \\
\text { decimals }\end{array}$ & $\begin{array}{l}\text { Neurologic } \\
\text { impairment }\end{array}$ & $\begin{array}{l}\text { Age at } \\
\text { testing } \\
\text { (years) }\end{array}$ & $\begin{array}{l}6 \mathrm{MWT} \\
\text { results } \\
\text { in } \\
\text { meters }\end{array}$ & $\begin{array}{l}\text { 6MWT } \\
\text { z-score }\end{array}$ \\
\hline
\end{tabular}

\begin{tabular}{|c|c|c|c|c|c|c|}
\hline \multicolumn{7}{|c|}{$\begin{array}{l}\text { Visual impairment + neurologic impairment } \\
\text { (12) }\end{array}$} \\
\hline 1 & $\begin{array}{l}\text { Leber congenital } \\
\text { amaurosis }\end{array}$ & $\begin{array}{l}\text { Legally } \\
\text { blind }\end{array}$ & Autism & 10.0 & 630 & -0.47 \\
\hline 4 & $\begin{array}{l}\text { Cerebral visual } \\
\text { impairment }\end{array}$ & $\begin{array}{l}0.6 \\
1.0 \\
0.3 \\
0.6\end{array}$ & $\begin{array}{l}\text { Additional } \\
\text { neurologic } \\
\text { impairments } \\
\text { inherent to } \\
\text { cerebral visual } \\
\text { impairment }\end{array}$ & $\begin{array}{l}8.6 \\
10.0 \\
11.0 \\
11.5\end{array}$ & $\begin{array}{l}550 \\
400 \\
600 \\
480\end{array}$ & $\begin{array}{l}-0.78 \\
-3.8 \\
-0.28 \\
-1.7\end{array}$ \\
\hline 3 & Hemianopsia & $\begin{array}{l}0.6 \\
0.6 \\
1.0\end{array}$ & $\begin{array}{l}\text { Cerebral } \\
\text { paresis }\end{array}$ & $\begin{array}{l}8.5 \\
11.5 \\
12.0\end{array}$ & $\begin{array}{l}680 \\
600 \\
430\end{array}$ & $\begin{array}{l}1.4 \\
-0.28 \\
-3.4\end{array}$ \\
\hline 1 & Stargardt & 0.1 & $\begin{array}{l}\text { Bardet Biedl } \\
\text { syndrome }\end{array}$ & 12.1 & 530 & -2.1 \\
\hline 1 & Optic hypoplasia & 0.1 & $\begin{array}{l}\text { Additionally } \\
\text { has mild global } \\
\text { developmental } \\
\text { delay }\end{array}$ & 10.5 & 520 & -2.5 \\
\hline 1 & $\begin{array}{l}\text { Cerebral visual } \\
\text { impairment and } \\
\text { optic atrophy }\end{array}$ & 0.25 & $\begin{array}{l}\text { Additional } \\
\text { neurologic } \\
\text { impairments } \\
\text { inherent to } \\
\text { cerebral visual } \\
\text { impairment }\end{array}$ & 10.5 & 510 & -2.0 \\
\hline 1 & Microphtalmia & 0.16 & $\begin{array}{l}\text { Microcephaly, } \\
\text { mild-moderate } \\
\text { developmental } \\
\text { delay }\end{array}$ & 11.2 & 680 & 0.81 \\
\hline
\end{tabular}




\section{REFERENCES}

1. Kousi M, Lehesjoki AE, Mole SE. Update of the mutation spectrum and clinical correlations of over 360 mutations in eight genes that underlie the neuronal ceroid lipofuscinoses. Hum Mutat 2012;33:42-63.

2. Ouseph MM, Kleinman ME, Wang QJ. Vision loss in juvenile neuronal ceroid lipofuscinosis (CLN3 disease). Ann NY Acad Sci 2016;1371:55-67.

3. Augustine EF, Mink JW. Juvenile NCL (CLN3 disease): emerging disease-modifying therapeutic strategies. Pediatr Endocrinol Rev 2016;13(suppl 1):655-662.

4. Geraets RD, Koh SY, Hastings ML, Kielian T, Pearce DA, Weimer JM. Moving towards effective therapeutic strategies for neuronal ceroid lipofuscinosis. Orphanet J Rare Dis 2016;11:40.

5. Kuper WFE, van Alfen C, Rigterink RH, Fuchs SA, van Genderen MM, van Hasselt PM. Timing of cognitive decline in CLN3 disease. J Inherit Metab Dis 2018;41:257-261.

6. $\quad$ ATS. ATS statement: guidelines for the six-minute walk test. Am J Respir Crit Care Med 2002;166:111-117.

7. Mylius CF, Paap D, Takken T. Reference value for the 6-Minute Walk Test in children and adolescents: a systematic review. Expert Rev Respir Med 2016;10:1335-1352.

8. World Health Organization. Change the Definition of Blindness. 2015. Available at: who. int/blindness/Change\%20the\%20Definition\%20of\%20Blindness.pdf. Accessed May 1, 2018.

9. Ulrich S, Hildenbrand FF, Treder U, et al. Reference values for the 6-Minute Walk Test in healthy children and adolescents in Switzerland. BMC Pulm Med 2013;13:49.

10. Snijders TAB, Bosker RJ. Multilevel Analysis: An Introduction to Basic and Advanced Multilevel Modeling, 2nd ed. Los Angeles: SAGE Publications; 2012.

11. Marshall FJ, de Blieck EA, Mink JW, et al. A clinical rating scale for Batten disease: reliable and relevant for clinical trials. Neurology 2005;65:275-279.

12. Santavuori P, Heiskala H, Westermarck T, Saino K, Moren R. Experience over 17 years with antioxidant therapy in Spielmeyer-Sjogren disease. Am J Hum Genet 1988;5:265-274.

13. Kristensen K, Lou HC. Central nervous system dysfunction as early sign of neuronal ceroid lipofuscinosis (Batten's disease). Dev Med Child Neurol 1983;25:588-590.

14. Lamminranta S, Aberg LE, Autti T, et al. Neuropsychological test battery in the follow-up of patients with juvenile neuronal ceroid lipofuscinosis. J Intellect Disabil Res 2001; 45:817.

15. Anderson GW, Goebel HH, Simonati A. Human pathology in NCL. Biochim Biophys Acta 2013;1832:1807-1826.

16. Licchetta L, Bisulli F, Fietz M, et al. A novel mutation of $C \ln 3$ associated with delayedclassic juvenile ceroid lipofuscinosis and autophagic vacuolar myopathy. Eur J Med Genet 2015;58:540-544.

17. Ostergaard JR, Rasmussen TB, Molgaard H. Cardiac involvement in juvenile neuronal ceroid lipofuscinosis (Batten disease). Neurology 2011;76:1245-1251. 



\section{CHAPTER DIFFERENTIATING OPHTHALMOLOGICAL CHARACTERISTICS OF CLN3 DISEASE (BATTEN DISEASE)}

Willemijn F.E. Kuper, Herman E. Talsma, Mary J. van Schooneveld, Jan Willem R. Pott, Barbara C.H. Huijgen, Gerard C. de Wit, Peter M. van Hasselt*, Maria M. van Genderen* *These authors contributed equally to this work Submitted

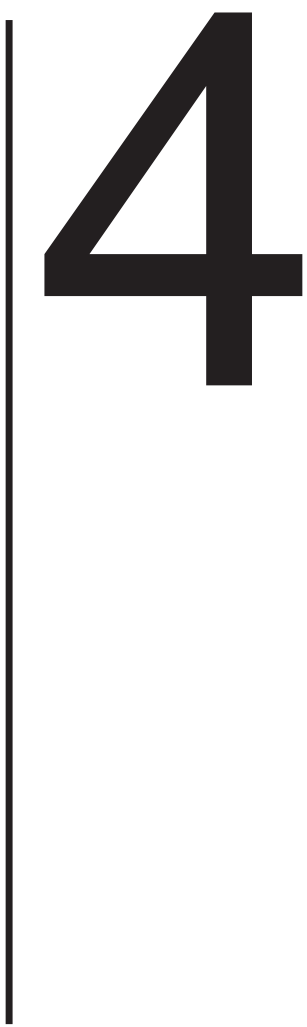




\section{ABSTRACT}

Purpose: To identify differentiating ophthalmological characteristics of CLN3 (Batten) disease, a devastating childhood metabolic disorder initially presenting similar to early-onset Stargardt disease (STGD1).

Methods: Medical chart review of 18 CLN3 disease patients and 20 early-onset STGD1 patients.

Results: Both diseases presented with visual acuity (VA) loss due to macular dystrophy at 5 to 10 years of age. VA in CLN3 disease decreased significantly faster than in STGD1 $(p=0.01)$. Color vision around diagnosis was often severely affected in CLN3 disease while unaffected or only mildly affected in STGD1. Optic disc pallor on fundoscopy and an abnormal nerve fiber layer on optical coherence tomography were common in CLN3 disease compared to generally unaffected in STGD1. In CLN3 disease, dark-adapted (DA) full field electroretinogram (ERG) responses were either absent, or electronegative. In early-onset STGD1, DA ERG responses were generally unaffected. None of the STGD1 patients had an electronegative ERG.

Conclusion: CLN3 disease and early-onset STGD1 may present apparently similar, but at the time of diagnosis the retina in CLN3 disease is more extensively and more severely affected. This results in more rapid VA loss, severe color vision abnormalities and abnormal DA ERG responses as the main differentiating features of CLN3 disease.

Key words: Batten disease; childhood retinal dystrophy; CLN3 disease; deep phenotyping; early-onset STGD1; early recognition

Summary statement: CLN3 disease is a devastating metabolic disorder initially presenting similar to early-onset Stargardt disease (STGD1), hindering timely recognition. This deep phenotyping study indicates that around diagnosis, rapid loss of vision, severe color vision deficiency and electronegative or absent dark-adapted ERG responses are differentiating ophthalmological features of CLN3 disease. 


\section{INTRODUCTION}

The majority of children presenting with vision loss due to macular dystrophy have early-onset Stargardt disease (STGD1) (OMIM \#248200), which is a retinaonly disease. ${ }^{1}$ However, a significant minority actually suffers from CLN3 disease (Batten disease) (OMIM \#204200), which is a devastating metabolic disease impacting both retina and brain, generally limiting life expectancy to their early twenties. ${ }^{2}$ Early distinction between CLN3 disease and earlyonset STGD1 is essential to tailor counseling, minimizing both a diagnostic odyssey in those with CLN3 disease, and unnecessary concerns in those with early-onset STGD1. However, at CLN3 disease onset, the brain is only subtly affected, which may make it difficult for an ophthalmologist to recognize the disorder. ${ }^{3-6}$ In addition, fundus features which are characteristic of STGD1, such as yellow-white flecks, are seen in only a minority of early-onset STGDT1 patients at presentation. ${ }^{1}$

In more frequent disorders, repeated exposure facilitates a pattern recognition process that allows clinicians to identify differentiating features that aid a correct diagnosis. The rareness of particularly CLN3 disease however precludes this naturally occurring learning curve complicating timely recognition even more. We hypothesized that recognition of a rare disorder could be improved by an in-depth analysis of all phenotypic abnormalities observed - also known as deep phenotyping -, compared to an apparently similar, but more frequent, disorder to identify differentiating phenotypic abnormalities. ${ }^{7}$ This way, pattern recognition could be made available for rare disorders as well. Although increasingly exome or even genome-wide analyses are being performed, the ability to recognize a rare disorder on clinical characteristics only is of ongoing relevance, particularly because in many countries genetic analysis is not yet routinely available or affordable. Not recognizing CLN3 disease at an early stage may have devastating consequences for affected children, as they will not get adequate support and treatment.

In this study, we explored the use of deep phenotyping to delineate defining ophthalmological characteristics of CLN3 disease at the time of diagnosis as compared to early-onset STGD1. The differentiating characteristics identified in this study could aid ophthalmologists in the early recognition of CLN3 disease. 


\section{METHODS}

\section{PATIENTS}

We retrieved the medical records of patients referred to Bartiméus for the diagnostic analysis of their vision loss, with a subsequent genetically confirmed diagnosis of either CLN3 disease (due to bi-allelic mutations in CLN3) or earlyonset STGD1 (due to bi-allelic mutations in ABCA4). ${ }^{1,2}$ Bartiméus is a tertiary diagnostic center for complex visual disorders and the national referral center for CLN3 disease. A waiver of requirement for ethical review was granted by The Medical Ethical Research Committee of the University Medical Center Utrecht.

\section{DATA COLLECTION}

We retrieved the clinical data from the medical records. From the clinical history, we retrieved the onset of vision loss and the presence of additional ophthalmological complaints (specifically night vision problems, photophobia) around diagnosis. We regarded the moment at which the first signs of rapid vision loss were noticed by the parents as the time point of onset $(T=0)$. If the onset was not reported, then the first rapid vision loss-related doctor visit was regarded as $\mathrm{T}=0$. From the validated clinical assessments we retrieved visual acuity (VA), color vision, and contrast sensitivity. To analyze vision loss over time, we included all VA measurements available from before, around, and after diagnosis. We retrieved the best corrected VA (BCVA) of both eyes (VODS) from each measurement. If the VA of the right eye (VOD) and the VA of the left eye (VOS) were described only separately, we used the BCVA in the better eye. Regarding color vision, we included the severity of a color vision deficiency if the Hardy Rand Rittler (HRR) test was included in the test battery. From ophthalmoscopy we retrieved imaging (fundus photography, fundus autofluorescence (FAF), and optical coherence tomography (OCT)), and full field electroretinography (ffERG). If available, we collected the original images and graphs in addition to the descriptive conclusion of these assessments. From the globally tested assessments, we retrieved peripheral visual field results (examined with confrontational techniques because of the young age of the patients).

Fundus photography and FAF were made using the Canon CX-1 camera. OCT images were obtained with RT-Vue (Optovue, Fremont, CA). ffERG recordings were performed in accordance with the guidelines of the International Society for Clinical Electrophysiology of Vision (ISCEV). ${ }^{8}$ To obtain the responses, 
Dawson-Trick-Litzkow (DTL) corneal electrodes were used. For flash stimulation, an Espion E3 System with ColorDome Stimulator (Diagnosys LLC, Cambridge, the UK) was used. From the extended ISCEV series measured at Bartiméus, we extracted the light adapted (LA), 3.0 and $30 \mathrm{~Hz}$ flicker responses and the dark adapted (DA) 0.01 and 3.0 responses. ${ }^{9}$

\section{DATA ANALYSIS}

Due to the retrospective nature of the study, VA was measured using different methods and thus reported in different units. To be able to analyze VA loss for all patients per diagnosis combined, we converted all VA measurements into decimals. We used multi-level modeling - that accounts for imbalanced repeated measurements - to assess the velocity of the vision loss per diagnosis. ${ }^{10}$ We analyzed the velocity of the vision loss during the complete follow-up (starting from $\mathrm{t}=0$ ) in general, as during the first year of follow-up specifically.

Retinal layer anatomy was analyzed by using the OCT MM6 scan that captured the foveal region (manually extracted from the 12 radial line scans by WK). The MM6 scan was used based on the recognition that the more commonly used line scans due to eccentric fixation often failed to capture the foveal region. The OCT scans of $n=9$ CLN3 disease patients and $n=13$ early-onset STGD1 patients were subjected to an assessment of the inner retina/nerve fiber layer (NFL) by three experienced ophthalmologists (MvS, JWP, and MvG) who were blinded for the underlying diagnosis. All three assessors were asked to classify the NFL of each OCT scan as normal - doubtfully (ab)normal - or abnormal.

\section{STATISTICAL ANALYSIS}

For multi-level modeling, MLwiN version 3.0.1 was used to investigate longitudinal changes in VODS over time (Centre for Multilevel Modelling, University of Bristol, Bristol, United Kingdom). An advantage of multilevel modeling over the traditional repeated-measurement approach is that all of the available results can be incorporated within the analysis, even if the number of measurements varies between the patients because of missing data, assuming that the missing data are random. A 2-level multilevel structure was used; level 1 represented repeated measures within participants, level 2 represented differences among patients. The predictor 'years since onset' was entered into the model to find the best model fit. The model fit was evaluated 
by comparing the deviance ( $\ln -2$ log likelihood) of the empty model with the final model.

The differences in age at onset, VA at onset and vision loss velocity (as denominated by the multi-level equation) between CLN3 disease and earlyonset STGD1 were calculated using the unpaired two-tailed t-test in Graphpad Prism version 8.0.1 (Graphpad Prism San Diego CA). P-values below 0.05 were considered statistically significant.

\section{RESULTS}

Between 1987 and 2019 a total of 38 patients with a subsequent genetically confirmed diagnosis of either CLN3 disease or early-onset STGD1 were referred to Bartiméus for the ophthalmological assessment of their vision loss. Of these, 18 were diagnosed with CLN3 disease and 20 with early-onset STGD1.

\section{VISION LOSS}

Patients with CLN3 disease exhibited a somewhat earlier onset of vision loss (mean age at onset of 6.4 years, range 5-8 years) than patients with earlyonset STGD1 (mean age at onset of 7.5 years, range $5-10$ years, $p=0.009$ ). In addition, there was a trend towards a worse VA at CLN3 disease presentation (mean VODS of 0.2 (comparable to 6/30 in Snellen $(\mathrm{m})$ ratios) in CLN3 disease compared to a mean VODS of 0.3 (comparable to $6 / 19$ in Snellen $(\mathrm{m})$ ratios) in STGD1, $p=0.12$ ). However, given their clinically significant overlap, neither age at onset nor VODS at presentation allowed to differentiate between earlyonset STGD1 or CLN3 disease. What clearly did discriminate CLN3 disease from early-onset STGD1, was the subsequent course of vision loss (Figure 1). Already within the first year after vision loss onset, VA decreased in CLN3 disease significantly faster $\left(\operatorname{VODS}_{1 y \mathrm{r}}=0,267-0,259^{\star}\right.$ part of the year since onset) than in early-onset STGD1 (VODS 1 rr $=0,411-0,357^{*}$ part of the year since onset) $(p=0.01)$. 
FIGURE 1 | VISION LOSS COURSE IN CLN3 DISEASE AND EARLY-ONSET STGD1

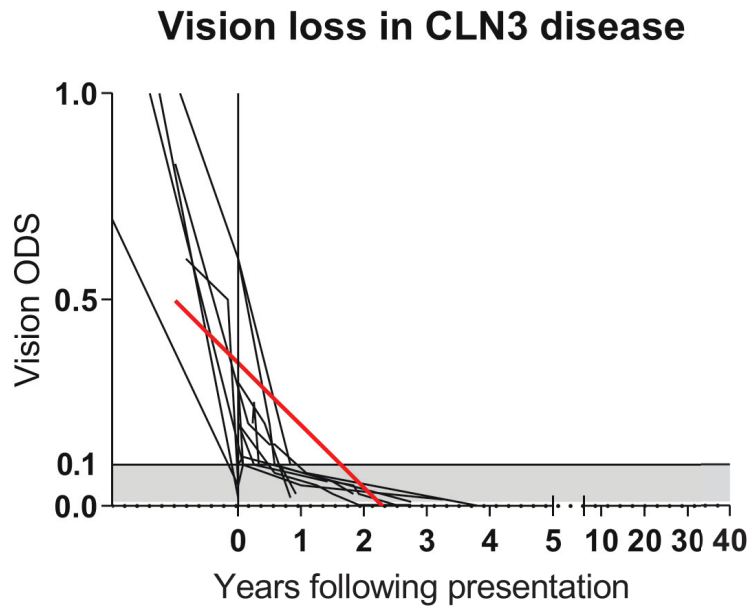

Vision loss in early onset STGD1

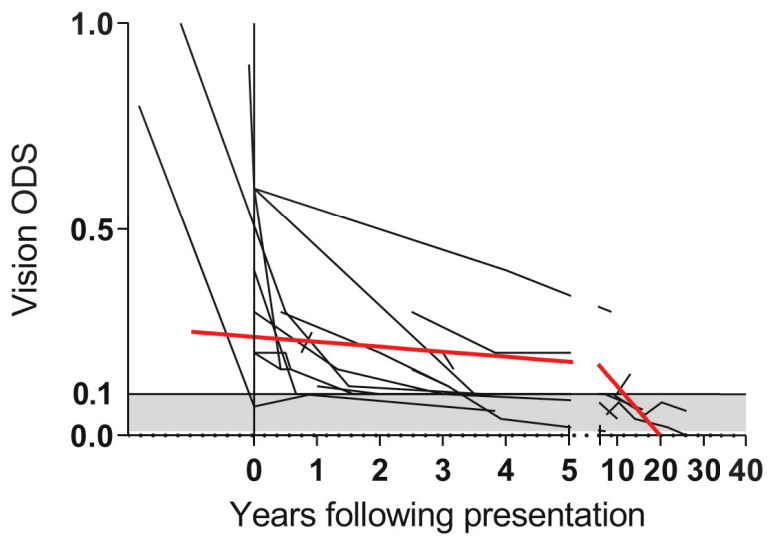

Visual acuity measurements in CLN3 disease compared to early-onset Stargardt disease (STGD1). Velocity coefficient calculations confirm similar VODS around presentation but subsequently a significantly faster decrease of visual acuity in CLN3 disease $\left(\right.$ VODS $_{\text {overall }}=0,347-0,151^{*}$ years since onset) compared to early-onset STGD1 $\left(V_{\text {VODS }}=0,347-0,151^{\star}\right.$ years since onset; $\left.p=0.02\right)$ for the complete follow-up period as shown in figure). In both cohorts, $V O D=$ VOS. 
COLOR VISION

Systematic assessment revealed that the severity of color vision deficiency allowed to distinguish most patients with CLN3 disease from those with earlyonset STGD1. Normal color vision was only seen in patients with early-onset STGD1. Conversely, severely affected color vision - most pronounced in redgreen color vision but also in tritan color vision - indicated that the patient was suffering from CLN3 disease (Figure 2 and Supplemental Table). Of note, in the only one (male) early-onset STGD1 patient exhibiting a severe RG color vision deficiency, a highly ordered Farnsworth D15 pattern was suggestive of a congenital deutan deficiency as relatively commonly occurring in males (Supplemental Table). ${ }^{11}$ 
FIGURE 2 | SEVERITY OF COLOR VISION DEFICIENCY AROUND DIAGNOSIS IN CLN3 DISEASE AND EARLY-ONSET STGD1

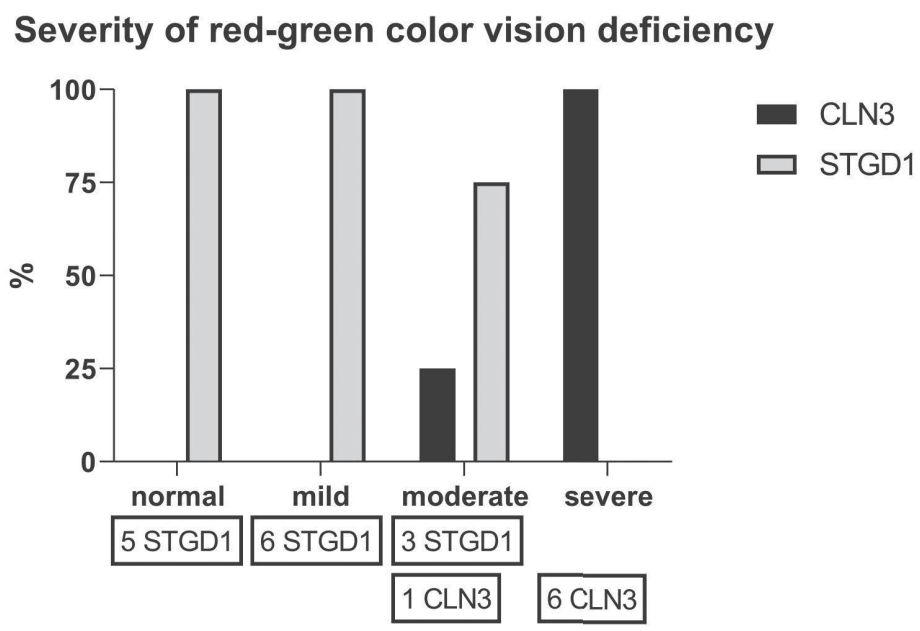

\section{Severity of tritan color vision deficiency}

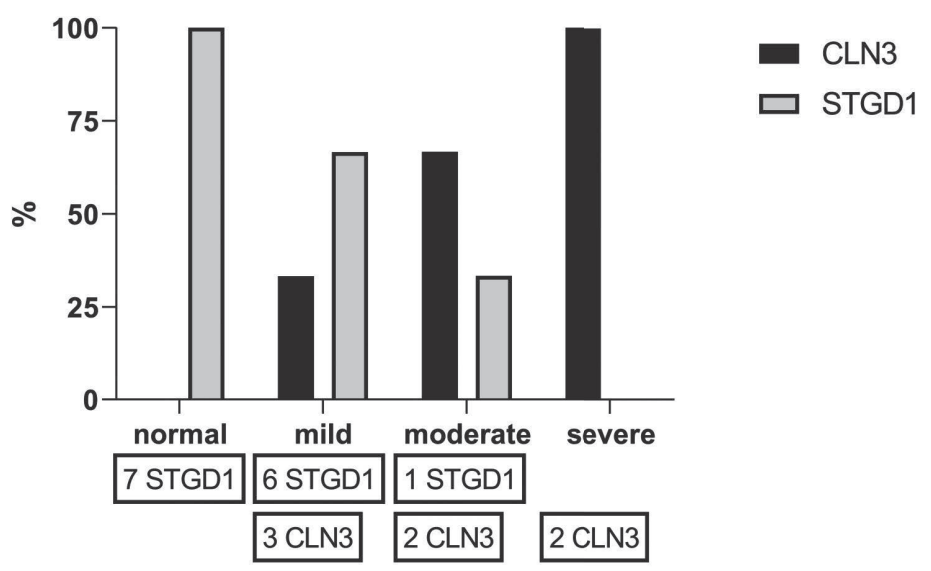

Red-green and tritan color vision deficiency in $n=7$ CLN3 disease patients and $n=14^{*}$ early-onset STGD1 patients tested with (at least) the HRR around diagnosis. *excluded from this figure is one (male) early-onset STGD1 patient with a suspected co-occurring congenital color vision deficiency (see also supplemental table). 


\section{FUNDOSCOPY}

Macular pigmentary changes were present in all CLN3 disease patients and the majority of early-onset STGD1 patients ( $n=17 / 20)$, but the presence of optic disc pallor and attenuated vessels proved somewhat more discriminatory: their presence was observed in the majority ( $n=12 / 17$ and $n=15 / 17$, respectively) of patients with CLN3 disease, being rare in patients with early-onset STGD1 ( $n=2 / 20$ and $n=2 / 19$, respectively) (Figure 3, Supplemental Table). Of note, as in previous studies on the early-onset type of STGD1, ${ }^{1,12}$ typical STGD1 fundus abnormalities - i.e. irregular yellow-white posterior fundus flecks - were only rarely seen in our early-onset STGD1 cohort, underscoring the difficulty to distinguish CLN3 disease from early-onset STGD1 in this initial phase.

OCT

Patients with CLN3 disease as well as early-onset STGD1 presented with macular atrophy on OCT. The optic disc pallor observed on fundoscopy suggested a CLN3 disease-specific early involvement of the inner retina. To further explore this hypothesis, we used the OCT scans to assess the different retinal layers. As expected, the outer photoreceptor related layers were severely affected in both CLN3 disease and early-onset STGD1. When focusing on the inner retina, the NFL was indeed often regarded abnormal in CLN3 disease but regarded as normal in early-onset STGD1 (Figure 3) (Table 1). 
FIGURE 3 | FUNDOSCOPY, FAF, AND OCT AROUND DIAGNOSIS IN CLN3 DISEASE AND EARLY-ONSET STGD1

CLN3 disease
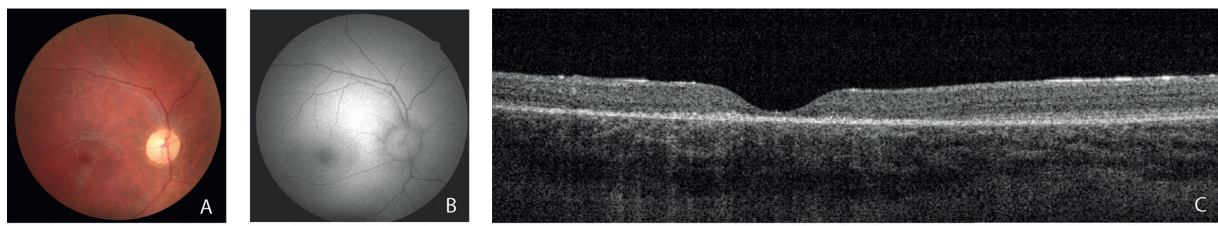

Early-onset STGD1
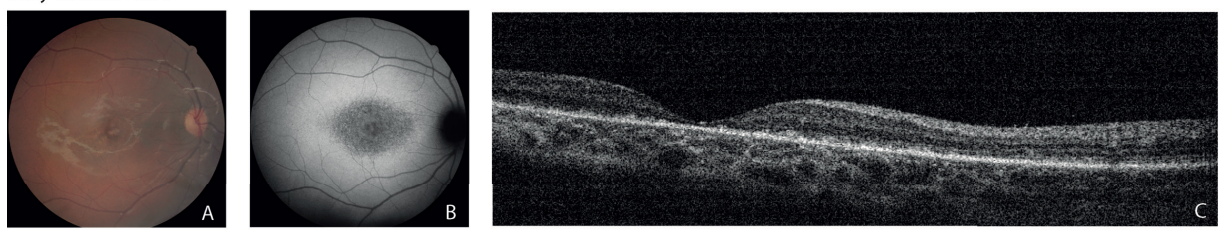

Healthy individual
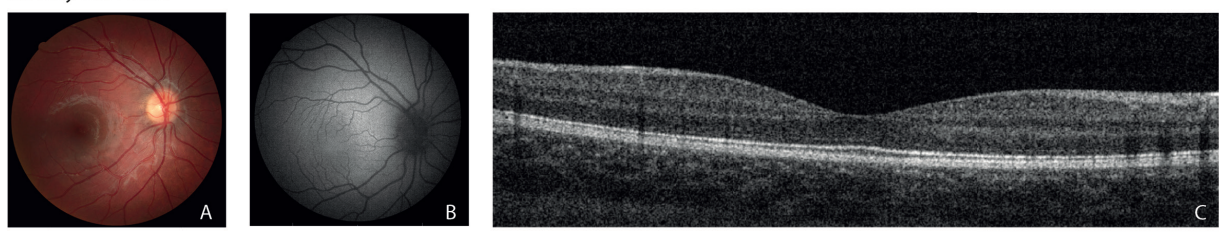

Representative fundoscopy (A), fundus autofluorescence (FAF) (B), and optical coherence tomography (OCT) (C), from $n=1$ CLN3 disease patient and $n=1$ early-onset STGD1 patient around diagnosis compared to $n=1$ age-matched healthy control.

TABLE 1 | QUALITATIVE ASSESSMENT OF THE NERVE FIBER LAYER IN CLN3 DISEASE AND EARLY-ONSET STGD1

\begin{tabular}{lllll}
\hline NFL & & Assessor 1 & Assessor 2 & Assessor 3 \\
\hline CLN3 & normal & 3 & 4 & \\
N=9 & doubt & 3 & 1 & 7 \\
& abnormal & 3 & 4 & 2 \\
& & & & \\
STGD1 & normal & 10 & 10 & 11 \\
N=13 & doubt & 2 & 1 & 2 \\
& abnormal & 1 & 2 & 2 \\
\hline
\end{tabular}

Qualitative assessment of the NFL performed by three independent assessors (experienced ophthalmologists) in $n=9$ CLN3 disease and $n=13$ early-onset STGD1 radial MM6 OCT scans made around diagnosis. 


\section{ERG}

In all CLN3 disease patients who underwent visual electrophysiology testing $(n=13)$, the DA ERG was described as markedly abnormal. In $n=7$ CLN3 disease patients we could retrieve the raw ERG responses to further zoom in on the different responses. In these, DA 0.01 responses were severely reduced or absent. The DA 3.0 bright flash responses showed a b-wave which was much smaller than the a-wave, also called an electronegative ERG. In addition, the photoreceptor-driven a-wave was reduced in $n=4 / 7$ patients. These results confirm that inner retinal dysfunction occurs (as represented in the b-wave) very early in CLN3 disease, in some patients preceding deterioration of the photoreceptors (as represented in the a-wave).13 While the DA ERG was severely reduced and electronegative in all CLN3 disease patients, the DA responses were unaffected $(n=8 / 11)$ or only mildly reduced $(n=3 / 11)$ in earlyonset STGD1. None of the early-onset STGD1 patients had an electronegative bright flash ERG (Figure 4 and Supplemental Table).

Of note, in our analysis, the LA ERG did not always discriminate CLN3 disease from early-onset STGD1, as it was within normal limits not only in 6 STGD1 patients, but also in 1 CLN3 patient. Thus, the DA ERG was necessary to distinguish CLN3 disease from early-onset STGD1 without any doubt. 
FIGURE 4 | LIGHT ADAPTED AND DARK ADAPTED ERG RESPONSES AROUND DIAGNOSIS IN CLN3 DISEASE AND EARLY-ONSET STGD1
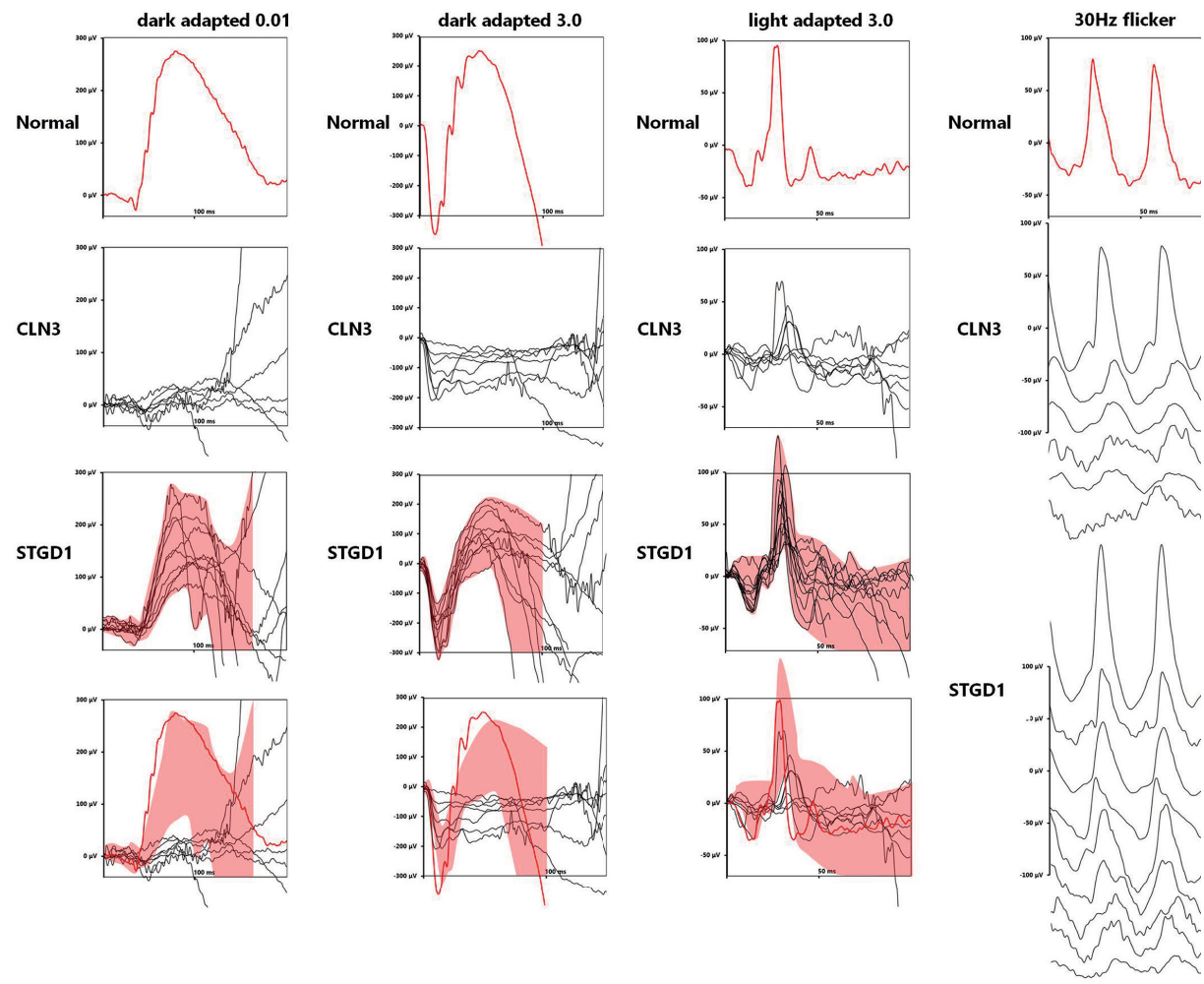

Light adapted (LA 3.0 and $30 \mathrm{~Hz}$ flicker) and dark adapted (DA 0.01 and 3.0) full-field ERG responses in $n=6$ CLN3 disease patients and $n=11$ early-onset STGD1 patients examined around diagnosis compared to $n=1$ age-matched healthy control. In the lower row the composite responses of CLN3 disease (individual black lines), STGD1 (red area) and normal subject (red line) are shown.

MULTIDIMENSIONAL ANALYSIS OF RETINAL INVOLVEMENT IN CLN3 DISEASE COMPARED TO EARLY-ONSET STGD1

Based on the diagnostic test results, CLN3 disease and early-onset STGD1 may seem very similar at presentation (Figure $5 \mathrm{~A}$ ), but can actually be distinguished well both on the extensiveness of retina involvement - that is the different components affected - as on the degree to which the different components were affected (Figure 5B). 
FIGURE 5 | MULTIDIMENSIONAL ANALYSIS OF RETINAL INVOLVEMENT IN CLN3 DISEASE COMPARED TO EARLY-ONSET STGD1
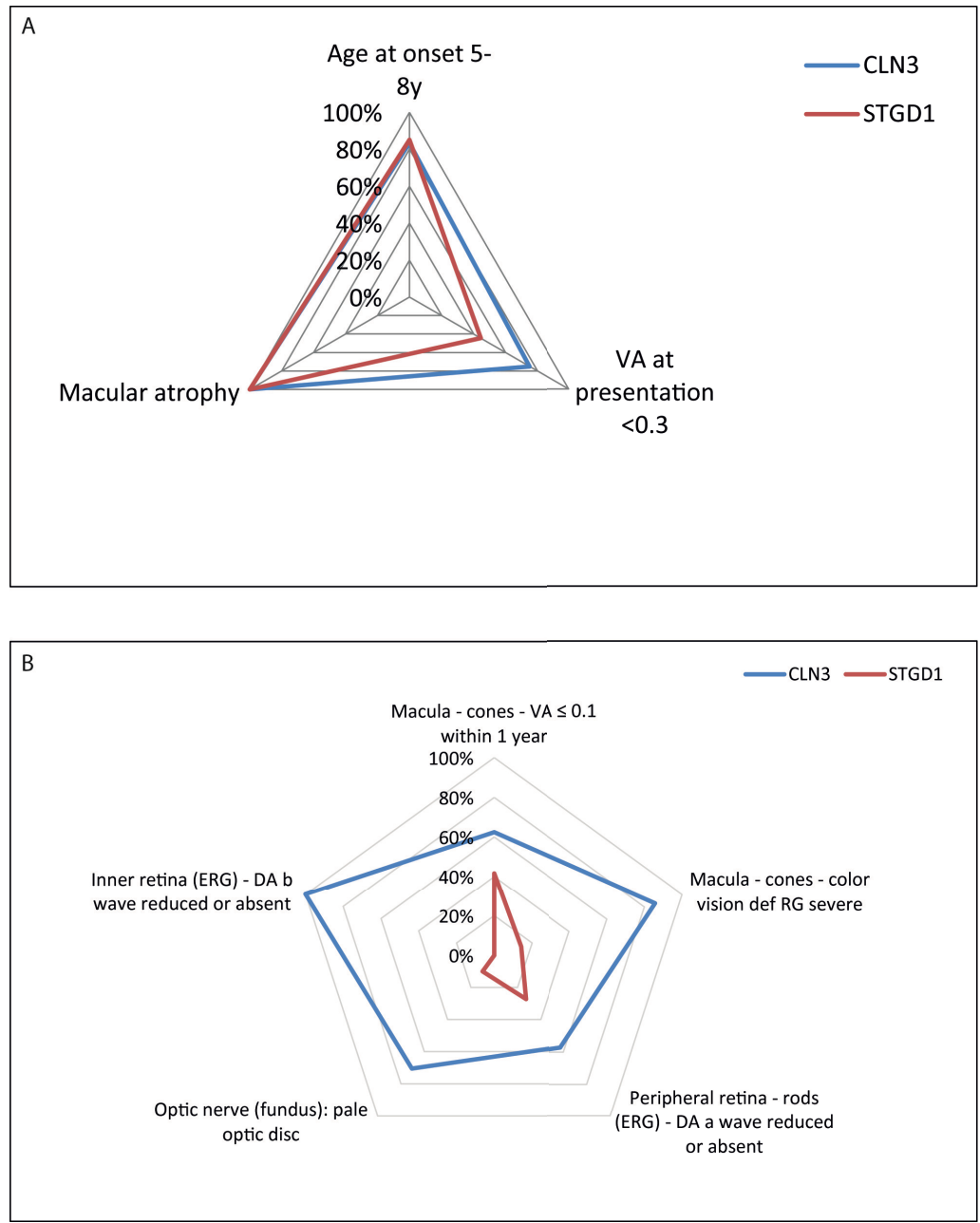

CLN3 disease and early-onset STGD1 present seemingly similar (A), but in-depth analysis of ophthalmological test results performed around diagnosis reveals that the retinal disease is in CLN3 disease already more extensive (B). In CLN3, more ocular components are involved compared to STGD1: not only photoreceptors (cones and rods) but also inner retina and optic nerve. In addition, the different components are more severely affected, e.g. a severe cone involvement resulting in a severely impaired VA and severe color vision deficiency around diagnosis.

Abbreviations: $V A$ = visual acuity; def = deficiency; $R G$ = red-green 


\section{DISCUSSION}

In this study, we explored whether we could aid the ophthalmologist to differentiate between a devastating metabolic disorder and a retina-only disease, despite their similar clinical presentation. Using a deep phenotyping approach, we unveiled that compared to early-onset STGD1, the retina is in CLN3 disease more extensively (involving both cones and rods, as optic nerve associated layers) and more severely affected, resulting in several consistent clinical and electrophysiological differentiating features of CLN3 disease. Three of these features most clearly aided us to distinguish CLN3 disease from early-onset STGD1: dramatically rapid loss of vision, severe color vision deficiency (both indicative of severe cone involvement) and absent or electronegative DA ERG responses (indicative of severe rod and optic nerve involvement). The ability to recognize a rare disorder such as CLN3 disease on clinical characteristics only is essential for speedy referral to a metabolic pediatrician or child neurologist, and for adequate rehabilitation, especially since (untargeted) genetic analysis is not always available or desirable. Patients with CLN3 disease often already face learning problems requiring more than only practical adjustments for their visual impariment. ${ }^{5}$

By making the implicit explicit, this approach made pattern recognition of the differentiating features of a rare retinal dystrophy available to the clinical practice in various contexts. We were able to show that while the visual acuity itself may not be clearly different between CLN3 disease and earlyonset STGD1 at presentation, the velocity of the vision loss clearly is different - already within the first year of follow-up. Thus, in circumstances when referral to a specialized center is not yet possible, re-examining visual acuity within a few months will already help to determine the probability of CLN3 disease as the underlying diagnosis. Next, we argue that color vision should be assessed with a test that specifies the severity of a color vision deficiency, such as the HRR. Our results indicate that in most children with vision loss due to macular atrophy, the probability of CLN3 disease as the underlying diagnosis can already reliably be determined based on the severity of a color vision deficiency. Finally, although it is more invasive than most of the other tests, our results clearly emphasize the relevance of the ERG. Importantly, to distinguish between CLN3 disease and early-onset STGD1, a dark adapted ERG must be included. This was in our study most strikingly exemplified by the one CLN3 disease patient with only a moderate (red-green) color vision deficiency and an unaffected light adapted ERG in whom thus only the - electronegative 
- dark adapted ERG responses were clearly outside the range of early-onset STGD1 and within the range of CLN3 disease. We advise to perform the ERG in these (young) children - who may in the case of CLN3 disease already suffer from subtle cognitive and/or behavioral problems - in a specialized center to maximize the chance to get (reliable) results. ${ }^{5,14,15}$

The apparent early involvement of the inner retina and optic nerve in CLN3 disease not only aids clinical recognition and understanding, but may also provide a clue towards understanding the function of the CLN3 protein, which has to date remained enigmatic. Early optic nerve atrophy is rare in most retinal dystrophies, but relatively common in mitochondrial disorders. ${ }^{16}$ In CLN3 disease, the predominant component of the accumulated storage material is the subunit c of mitochondrial F0 ATP synthase, a major component of the inner mitochondrial membrane. It has been postulated that the CLN3 protein functions in a specific part of mitochondrial turnover. ${ }^{17}$ When the CLN3 protein is deficient, particularly those areas that require a highly efficient mitochondrial turnover - such as the optic nerve associated layers - might deteriorate. Cln3 was found to primarily localize to the inner retina layers in mice and when deficient optic nerve atrophy was an early feature, ${ }^{16-19}$ but ongoing difficulties with CLN3 antibody specificity preclude definite conclusions on CLN3 location and function. ${ }^{20}$

To the best of our knowledge, we provide the most extensive overview of the ophthalmological characteristics early in CLN3 disease described so far. Previous reports on ocular abnormalities in CLN3 disease generally discussed either relatively old patients years after diagnosis or small patient numbers, without or with only few control patients with an isolated retinal disease. ${ }^{21-26}$ The extensive nature of the current study allowed us to gain new insights, yet still within limits. The eccentric fixation and limited cooperation of the children led us to use MM6 OCT images in this study. While very useful to identify retinal dystrophy with macular atrophy, these images are less useful for in-depth analysis of retinal layers in these children. This may explain why we did not corroborate a CLN3 disease-specific macular striation pattern, ${ }^{23,26}$ although we did find an apparent CLN3 disease-specific early inner retina/ NFL involvement. ${ }^{24}$

The phenotype of CLN3 disease is distributed among a spectrum. While most patients suffer from classical CLN3 disease at the severe end of the spectrum, 
protracted phenotypes have been described as well. In protracted CLN3 disease, neurodegeneration may be delayed, but the retinal phenotype is similar as in classical CLN3 disease underscoring the importance of CLN3 in the retina. Interestingly, recent research has revealed a new phenotypic entity at the mild end of the phenotypic spectrum, named CLN3 associated (isolated) retinal degeneration, in which neurodegeneration may never occur at all, and which has a different retinal phenotype as well. ${ }^{27-29}$ Whereas in classical and protracted CLN3 disease, the retinal phenotype is characterized by a conerod dystrophy with childhood onset rapidly deteriorating to blindness, in the CLN3-retina-only phenotype rather a rod-cone dystrophy is seen with adolescence-adult onset more slowly progressing over years to decades. Whereas the contents of our study may only be applicable to classical up to and including protracted CLN3 disease, the approach of our study may similarly be used to improve recognition and understanding of CLN3- associated retinal degeneration.

In conclusion, this study unveiled defining characteristics that aid clinicians in differentiating between two rare but clinically significant disorders of the retina - CLN3 disease and early-onset STGD1 - based on their presenting clinical characteristics only. This differentiation allows early identification of children with CLN3 disease essential for adequate referral, counselling and rehabilitation. 


\section{SUPPLEMENTAL TABLE}

OVERVIEW OF OPHTHALMOLOGICAL EXAMINATIONS AROUND DIAGNOSIS IN CLN3 DISEASE AND EARLY-ONSET STGD1

\begin{tabular}{|c|c|c|}
\hline & CLN3 disease $\left(n=18^{\star}\right)$ & Early-onset STGD1 $\left(n=20^{\star}\right)$ \\
\hline \multicolumn{3}{|c|}{ Visual functions - validated clinical assessments } \\
\hline $\begin{array}{l}\text { Color vision - } \\
\text { red/green }\end{array}$ & $\begin{array}{l}\text { With severity testing }(n=7) \\
\text { Unaffected in } 0 \\
\text { Mildly affected in } 0 \\
\text { Moderately affected in } 1 \\
\text { Severely affected in } 6\end{array}$ & $\begin{array}{l}\text { With severity testing }(\mathrm{n}=15) \\
\text { Unaffected in } 5 \\
\text { Mildly affected in } 6 \\
\text { Moderately affected in } 3 \\
\text { Severely affected in } 1^{\star \star}\end{array}$ \\
\hline & $\begin{array}{l}\text { Without severity testing }(n=4) \\
\text { Unaffected in } 0 \\
\text { At least mildly affected in } 4\end{array}$ & $\begin{array}{l}\text { Without severity testing }(n=2) \\
\text { Unaffected in } 1 \\
\text { At least mildly affected in } 1\end{array}$ \\
\hline $\begin{array}{l}\text { Color vision - } \\
\text { tritan }\end{array}$ & $\begin{array}{l}\text { With severity testing }(n=7) \\
\text { Unaffected in } 0 \\
\text { Mildly affected in } 3 \\
\text { Moderately affected in } 2 \\
\text { Severely affected in } 2\end{array}$ & $\begin{array}{l}\text { With severity testing }(n=15) \\
\text { Unaffected in } 8 \\
\text { Mildly affected in } 6 \\
\text { Moderately affected in } 1 \\
\text { Severely affected in } 0\end{array}$ \\
\hline Contrast vision & Impaired in $5 / 5$ & Impaired in $6 / 12$ \\
\hline \multicolumn{3}{|c|}{ Imaging and electrophysiology } \\
\hline \multicolumn{3}{|l|}{ Fundus } \\
\hline Macula & Affected in 17/17 & Affected in $17 / 20^{\star \star \star}$ \\
\hline Periphery & Affected in 9/14 & Normal in $12 / 15$ \\
\hline Optic Disc & Pallor in $12 / 17$ & $\begin{array}{l}\text { Normal in } 18 / 20 \\
\text { Slight temporal pallor in } 2 / 20\end{array}$ \\
\hline Vessels & Attenuated in 15/17 & Normal in $17 / 19$ \\
\hline
\end{tabular}

Fundus auto fluorescence (FAF)

Hypo-AF macula in 5/5 Hypo-AF macula in $7 / 7$

Optical coherence tomography (OCT)

Thinned retina with atrophic Thinned retina with atrophic fovea in $9 / 9$ fovea in $13 / 13$

\section{Electroretinography (ERG)}

\begin{tabular}{lll}
\hline Light adapted & Unaffected in $1 / 13$ & Unaffected in 6/11 \\
responses & Reduced in $12 / 13$ & Reduced in $5 / 11$
\end{tabular}


SUPPLEMENTAL TABLE | CONTINUED

\begin{tabular}{|c|c|c|}
\hline & CLN3 disease $\left(n=18^{\star}\right)$ & Early-onset STGD1 $\left(n=20^{\star}\right)$ \\
\hline \multirow{3}{*}{$\begin{array}{l}\text { Dark adapted } \\
\text { responses }\end{array}$} & Unaffected in 0 & Unaffected in $8 / 11$ \\
\hline & Reduced in $13 / 13$ & Reduced in $3 / 11$ \\
\hline & $\begin{array}{l}\text { Electronegative or absent DA } \\
\text { responses in } 13 / 13\end{array}$ & $\begin{array}{l}\text { Electronegative or absent DA } \\
\text { responses in } 0 / 11\end{array}$ \\
\hline \multicolumn{3}{|l|}{ Other } \\
\hline \multicolumn{3}{|c|}{ Visual functions - globally assessed } \\
\hline $\begin{array}{l}\text { Peripheral field } \\
\text { vision }\end{array}$ & Impaired in $6 / 10$ & Unaffected in $12 / 13$ \\
\hline \multicolumn{3}{|c|}{ Visual functions - anamnestically assessed } \\
\hline Night vision & Impaired in $10 / 13$ & Unaffected in $12 / 12$ \\
\hline Photophobia & Present in 4/9 & Present in $5 / 13$ \\
\hline \multicolumn{3}{|c|}{$\begin{array}{l}\text { * total number of patients included; per test the number of patients of whom results o } \\
\text { this test could be retrieved is described } \\
\text { ** panel D15 appearance suggestive of a congenital deutan deficiency (male patient } \\
\text { no explanatory family history of congenital color vision deficiency). } \\
\text { *** on OCT, all early-onset STGD1 patients displayed macular atrophy }\end{array}$} \\
\hline
\end{tabular}




\section{REFERENCES}

${ }^{1}$ Lambertus S, van Huet RA, Bax NM et al. Early-onset stargardt disease: phenotypic and genotypic characteristics. Ophthalmology. 2015;122(2):335-44.

${ }^{2}$ Mole SE, Cotman SL. Genetics of the neuronal ceroid lipofuscinoses (Batten disease). Biochim Biophys Acta. 2015;1852(10 Pt B):2237-41.

${ }^{3}$ Bohra LI, Weizer JS, Lee AG, Lewis RA. Vision loss as the presenting sign in juvenile neuronal ceroid lipofuscinosis. J Neuroophthalmol. 2000; 20(2):111-115.

${ }^{4}$ Ouseph MM, Kleinman ME, Wang QJ. Vision loss in juvenile neuronal ceroid lipofuscinosis (CLN3 disease). Ann N Y Acad Sci. 2016;1371(1):55-67.

${ }^{5}$ Kuper WFE, van Alfen C, Rigterink RH et al. Timing of cognitive decline in CLN3 disease. J Inherit Metab Dis. 2018;41(2):257-261.

${ }^{6}$ Kuper WFE, van Alfen C, van Eck L et al. Motor function impairment is an early sign of CLN3 disease. Neurology. 2019;93(3):e293-e297.

${ }^{7}$ Robinson PN. Deep phenotyping for precision medicine. Hum Mutat. 2012;33(5):777-80.

${ }^{8}$ McCulloch DL, Marmor MF, Brigell MG et al. ISCEV standard for full-field clinical electroretinography (2015 update). Doc Ophthalmol 2015;130(1):1-12.

${ }^{9}$ Brouwer AH, de Wit GC, Ten Dam NH et al. Prolonged cone b-wave on electroretinography is associated with severity of inflammation in non-infectious uveitis. Am J Ophthalmol. 2019 pii: S0002-9394(19)30261-2.

${ }^{10}$ Snijders TAB, Bosker RJ. Multilevel Analysis: An Introduction to Basic and Advanced Multilevel Modeling, 2nd ed. Los Angeles: SAGE Publications; 2012.

${ }^{11}$ Simunovic MP. Colour vision deficiency. Eye (Lond). 2010;24(5):747-55.

${ }^{12}$ Bax NM, Lambertus S, Cremers FPM et al. The absence of fundus abnormalities in Stargardt disease. Graefes Arch Clin Exp Ophthalmol. 2019;257(6):1147-1157.

${ }^{13}$ Audo I, Robson AG, Holder GE, Moore AT. The negative ERG clinical phenotypes and disease mechanisms of inner retinal dysfunction. Surv Ophthalmol. 2008;53(1):16-40.

${ }^{14}$ Spalton DJ, Taylor DS, Sanders MD. Juvenile batten's disease: An ophthalmological assessment of 26 patients. Br J Ophthalmol. 1980; 64(10):726-732.

${ }^{15}$ van Genderen MM, Riemslag F, Jorritsma F et al. The key role of electrophysiology in the diagnosis of visually impaired children. Acta Ophthalmol Scand. 2006 ;84(6):799-806.

${ }^{16}$ Carelli V, Ross-Cisneros FN, Sadun AA. Mitochondrial dysfunction as a cause of optic neuropathies. Progress in retinal and eye research. Prog Retin Eye Res. 2004;23(1):53-89.

${ }^{17}$ Katz ML, Gao CL, Prabhakaram M et al. Immunochemical localization of the Batten disease (CLN3) protein in retina. Invest Ophthalmol Vis Sci. 1997;38(11):2375-86.

${ }^{18}$ Sappington RM, Pearce DA, Calkins DJ. Optic nerve degeneration in a murine model of juvenile ceroid lipofuscinosis. Invest Ophthalmol Vis Sci. 2003;44(9):3725-31.

${ }^{19}$ Weimer JM, Custer AW, Benedict JW et al. Visual deficits in a mouse model of Batten disease are the result of optic nerve degeneration and loss of dorsal lateral geniculate thalamic neurons. Neurobiol. Dis. 2006; 22: 284-293.

${ }^{20}$ Nelson T, Pearce DA, Kovács AD. Lack of specificity of antibodies raised against CLN3, the lysosomal/endosomal transmembrane protein mutated in juvenile Batten disease. Biosci Rep. 2017 ;37(6).

${ }^{21}$ Hainsworth DP, Liu GT, Hamm CW, Katz ML. Funduscopic and angiographic appearance in the neuronal ceroid lipofuscinoses. Retina. 2009;29(5):657-68.

${ }^{22}$ Eksandh LB, Ponjavic VB, Munroe PB et al. Full-field ERG in patients with Batten/SpielmeyerVogt disease caused by mutations in the CLN3 gene. Ophthalmic Genet. 2000;21(2):69-77. 
${ }^{23}$ Dulz S, Wagenfeld L, Nickel $\mathrm{M}$ et al. Novel morphological macular findings in juvenile CLN3 disease. Br J Ophthalmol. 2016; 100(6):824-8.

${ }^{24}$ Preising MN, Abura M, Jäger M et al. Ocular morphology and function in juvenile neuronal ceroid lipofuscinosis (CLN3) in the first decade of life. Ophthalmic Genet. 2017;38(3):252259.

${ }^{25}$ Hansen MS, Hove MN, Jensen H, Larsen M. OPTICAL COHERENCE TOMOGRAPHY IN JUVENILE NEURONAL CEROID LIPOFUSCINOSIS. Retin Cases Brief Rep. 2016;10(2):137-9.

${ }^{26}$ Wright GA, Georgiou M, Robson AG et al. Juvenile Batten Disease (CLN3): Detailed Ocular Phenotype, Novel Observations, Delayed Diagnosis, Masquerades, and Prospects for Therapy. Ophthalmol Retina. 2019 Nov 13. pii: S2468-6530(19)30629-3.

${ }^{27}$ Wang F, Wang H, Tuan HF et al. Next generation sequencing-based molecular diagnosis of retinitis pigmentosa: identification of a novel genotype-phenotype correlation and clinical refinements. Hum Genet. 2014;133(3):331-45.

${ }^{28} \mathrm{Ku}$ CA, Hull S, Arno G et al. Detailed Clinical Phenotype and Molecular Genetic Findings in CLN3-Associated Isolated Retinal Degeneration. JAMA Ophthalmol. 2017;135(7):749-760.

${ }^{29}$ Kuper WFE, van Alfen C, van Eck L et al. A case of unexpected adult-onset neurologic decline in CLN3-associated retinal degeneration. JAMA Ophthalmol. 2017;135(12):1451-1453. 



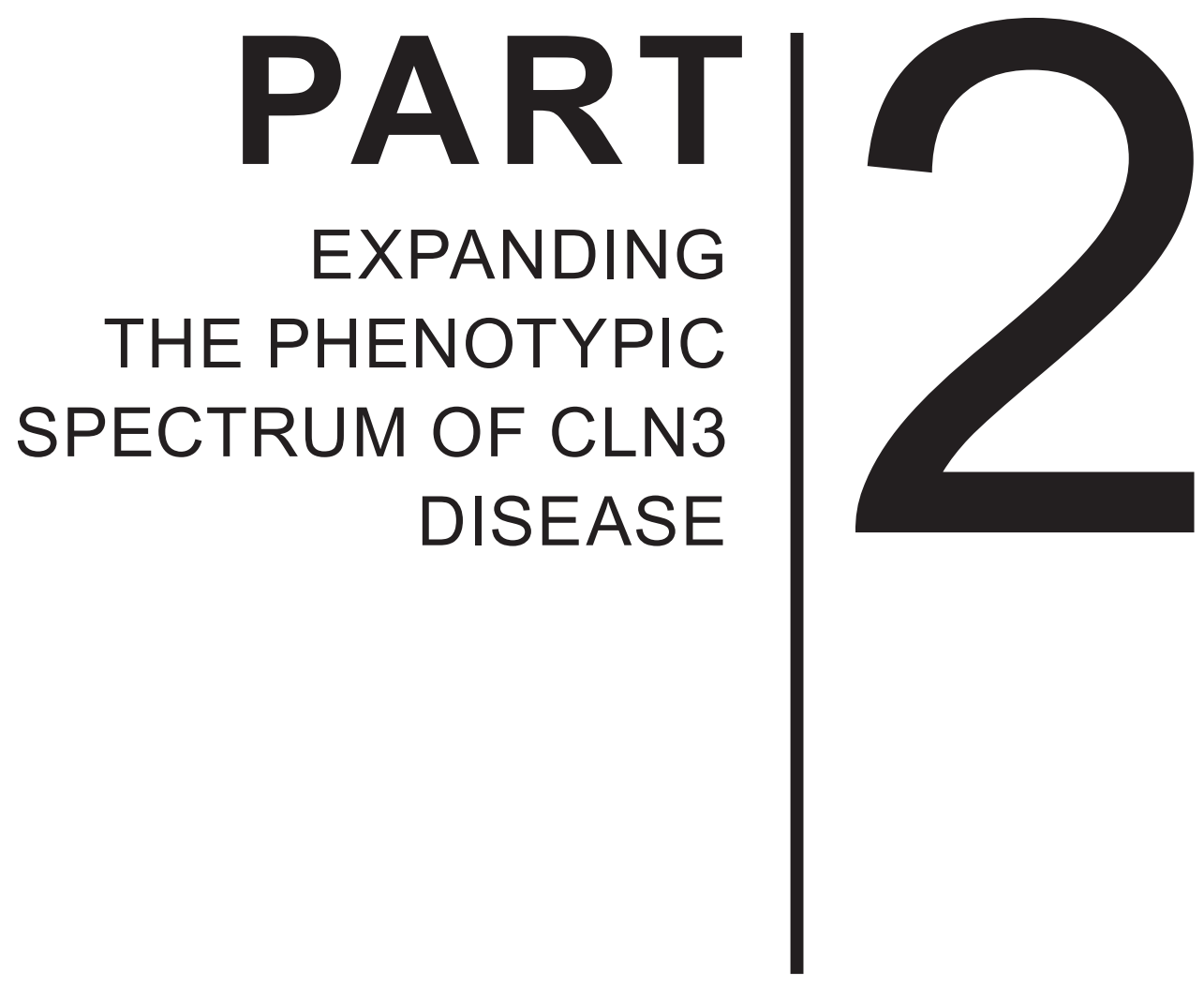





\section{CHAPTER \\ THE C.1A>C START CODON MUTATION IN CLN3 IS ASSOCIATED WITH A PROTRACTED DISEASE COURSE}

Willemijn F.E. Kuper, Claudia van Alfen, Linda van Eck, Stella A. de Man, Marjolein H. Willemsen, Koen L.I. van Gassen, Monique Losekoot, Peter M. van Hasselt JIMD Rep. 2020 Feb 7;52(1):23-27

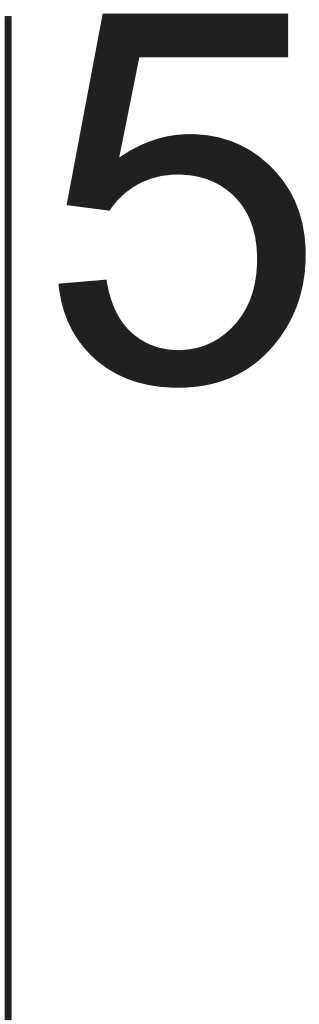




\section{ABSTRACT}

Background: CLN3 disease is a disorder of lysosomal homeostasis predominantly affecting the retina and the brain. The severity of the underlying mutations in CLN3 particularly determine onset and course of neurological deterioration. Given the highly conserved start codon code among eukaryotic species, we expected a variant in the start codon of CLN3 to give rise to the classical, i.e. severe, phenotype.

Case series: We present three patients with an identical CLN3 genotype (compound heterozygosity for the common $1 \mathrm{~kb}$ deletion in combination with a c. $1 \mathrm{~A}>\mathrm{C}$ start codon variant) who all displayed a more protracted phenotype than expected. While their retinal phenotype was similar to as expected in classical CLN3 disease, their neurological phenotype was delayed. Two patients had an early onset of cognitive impairment, but a particularly slow deterioration afterwards without any obvious motor impairment. The third patient also had a late onset of cognitive impairment.

Conclusions: Contrasting our initial expectations, patients with a start codon variant in CLN3 may display a protracted phenotype. Future work will have to reveal the exact mechanism behind the assumed residual protein synthesis, and determine whether this may be eligible to start codon targeted therapy.

Synopsis: Counter-intuitively, a variant in the CLN3 start codon may give rise to a protracted phenotype.

Key Words: CLN3; start codon; translation initiation codon; protracted phenotype; counseling 


\section{INTRODUCTION}

The start codon is responsible for the initiation of transcription and errors herein are intuitively expected to result in the absence of a functional protein (Kozak, 1991). As phenotype correlates with genotype, a severe phenotype is expected. In our clinic, we identified a patient with the common $1 \mathrm{~kb}$ deletion and a c.1A $>C$ start codon variant in CLN3 (Kousi et al, 2012). CLN3 disease (OMIM \#204200) is a neurodegenerative lysosomal storage disorder predominantly affecting the retina and the brain. In the classical phenotype - deriving from two truncating mutations expected to completely abolish CLN3 protein synthesis -, neurodegeneration already starts and progresses in childhood limiting life span of patients to their late teens/early twenties (Kuper et al, 2018; Kuper et al, 2019; Kousi et al, 2012). Contrary to our expectations, the patient displayed a protracted phenotype - defined as deriving from at least one 'mild' (missense) mutation still allowing some residual protein synthesis associated with a delayed neurological phenotype (Kousi et al, 2012). The milder phenotype could not be explained by the $1 \mathrm{~kb}$ deletion on the other allele, as this common founder mutation severely affects CLN3 protein synthesis generally hypothesized to completely abolish CLN3 protein function (Mirza et al, 2019; Munroe et al, 1997). Indeed, although some degree of phenotypic heterogeneity is seen, homozygosity for the $1 \mathrm{~kb}$ deletion present in around $75 \%$ of CLN3 disease patients - is consequently associated with the classical phenotype (Adams et al, 2010; Lebrun et al, 2011; Kousi et al, 2012; Munroe et al, 1997).

In this report, we assessed the hypothesis that the c. $1 \mathrm{~A}>\mathrm{C}$ variant in $C L N 3$ is responsible for a protracted CLN3 disease phenotype by successfully searching for additional patients harboring this genotype and delineating their disease course.

\section{CASE SERIES}

\section{CASE 1}

This 14-year old boy is the only child born to unrelated, Dutch parents. In his early childhood, a mild developmental delay was noticed, particularly concerning behavioral abnormalities, which led to the diagnosis of an autism spectrum disorder. Around the age of 5 years, a rapid decrease of vision was 
noticed and following ophthalmological examinations a cone-rod dystrophy was identified. Due to the combination of a retinal dystrophy with a mild developmental delay, the suspicion of CLN3 disease was raised. At the age of 6 years, the diagnosis of CLN3 disease was confirmed based on compound heterozygosity for the $1 \mathrm{~kb}$ deletion and a $\mathrm{c} .1 \mathrm{~A}>\mathrm{C}$ start codon variant in $C L N 3$. Ever since presentation, the patient has remained essentially stable, without clear signs of cognitive or motor deterioration. Regarding cognitive functions, his total IQ (TIQ) of 71 around diagnosis at 6 years of age has only declined to a verbal IQ (VIQ) of 67 at 12 years of age. Although TIQ and VIQ (at 12 years a TIQ could no longer be assessed since the patient is completely blind) are not directly interchangeable, this does suggest some degree of cognitive stabilization. More clearly, regarding motor function, this patient has walked a near-normal, stable 6-minute walk test (6MWT) distance in the past years - contrasting the early and continuous decline seen in (classical) CLN3 disease (Kuper et al, 2019) (Figure 1). Recently, at the age of 13 years, he has experienced his first generalized tonic-clonic seizure.

FIGURE 1 | 6MWT PERFORMANCE IN A PATIENT WITH THE 1.A $>$ C START CODON MUTATION IN CLN3 COMPARED TO CLASSICAL CLN3 DISEASE

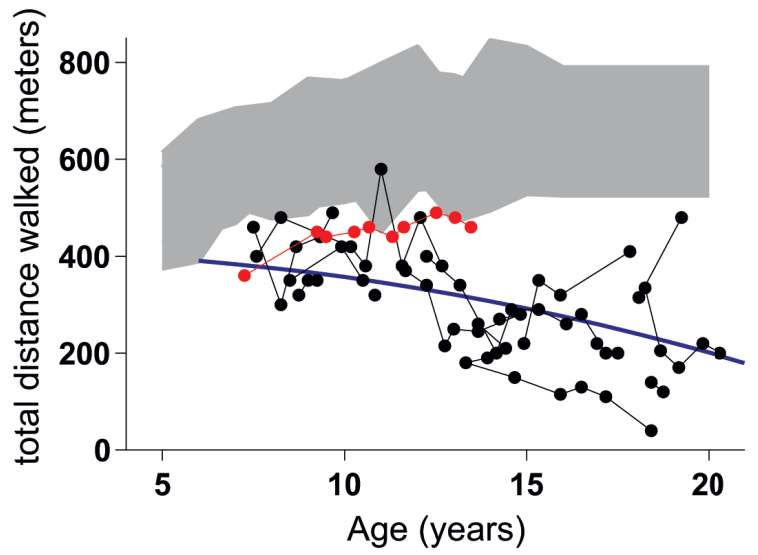

Figure adjusted from Kuper et al, 2019. The red dots show the 6-minute walk test (6MWT) performance of case 1 indicating a stable, near-normal, walking distance. This increasingly deviates from the classical CLN3 disease decline as depicted by the black dots (individual test results) and the blue line (overall velocity of the 6MWT distance decline over time). The grey area shows the 6MWT reference values in healthy, sighted children (+-2 SD). 


\section{CASE 2}

This woman, currently in her mid-twenties, was born to unrelated, Dutch parents. Her younger sister and brother are healthy. Of note, two far relatives had died due to CLN3 disease. No further details, particularly no genetic information, could be retrieved. Her early development was reportedly normal. Around the age of 7 years a rapid decrease in her vision was noticed. A retinal dystrophy affecting the macula was found, which, in combination with the suggested family history of CLN3 disease, led to the identification of two compound heterozygous disease causing variants in CLN3 (the $1 \mathrm{~kb}$ deletion $+c .1 \mathrm{~A}>\mathrm{C}$ start codon variant). Following the diagnosis of CLN3 disease, her parents were counselled on the expected disease course in classical CLN3 disease. They therefore expected that she would decline severely in the following years resulting in premature death around 20 years of age. In the following years however, psychomotor deterioration was profoundly slower than expected. While she currently, at the age of 24 , has exhibited some degree of deterioration, the deterioration is limited mainly concerning short term memory, concentrating on multiple tasks and word finding. She is still able to perform simple tasks on a farm specialized in care for the mentally handicapped. Similarly, and perhaps even more strikingly, her motor function still seems preserved. She does not experience any extrapyramidal symptoms: her speech is still clear and she still walks independently without the need for a wheelchair. Only since the age of 23 years, she has started to suffer from generalized tonic-clonic seizures.

\section{CASE 3}

This woman was the first child born to unrelated, Dutch parents with a healthy younger sister. Following a normal early development, a rapid loss of vision was noticed at the age of 7 years initially attributed to juvenile macular degeneration (Stargardt disease). However, after her first generalized tonicclonic seizure at 11 years of age she was diagnosed with CLN3 disease based on a skin biopsy and genetic analysis, although only one disease causing variant (the common $1 \mathrm{~kb}$ deletion) could be identified at that time. Despite the expected deterioration, her cognitive and motoric abilities remained preserved for years. With practical adjustments for her visual handicap, she was able to follow regular level education and even to graduate from high school at the age of 18 years which has - to our knowledge - not been reported 
in classical CLN3 disease and which strongly implies an (above) average IQ until her late teens. Around her final year at high school however, a decrease in her learning abilities and short term memory became increasingly obvious. In the following years, she quickly deteriorated both in her cognitive and motoric abilities. From her early twenties onwards, she lost the ability to walk and her speech became unintelligible. She died just before the age of 26 years. After she passed away, carrier ship analysis within the family led to the identification of the second variant - the $\mathrm{C} .1 \mathrm{~A}>\mathrm{C}$ start codon variant - in CLN3.

\section{DISCUSSION}

We report on three CLN3 disease patients compound heterozygous for the common $1 \mathrm{~kb}$ deletion and a c. $1 \mathrm{~A}>\mathrm{C}$ start codon variant in CLN3 who all displayed a protracted phenotype. The CLN3 protein is a transmembrane protein with yet unresolved function. In the absence of a specific antibody (precluding reliable residual protein analysis) and/or an assay measuring residual activity we focused on the clinical underpinning (Nelson et al, 2017).

Based on this milder clinical presentation, we hypothesize that the c. $1 \mathrm{~A}>\mathrm{C}$ variant in CLN3 does not completely abolish CLN3 protein synthesis. Interestingly, although start codon variants are often associated with a severe disease course (Sargiannidou et al, 2015; Won et al, 2019), there are several reports of disease causing start codon variants describing a milder phenotype (Chen et al, 2016; Pittis et al, 2004; Sadhwani et al, 2018). The residual protein synthesis in case of such a start codon variant may be explained by the production of a shorter but still partly functional protein because of the use of an alternative downstream start codon, either in the mutated transcript itself or in alternatively spliced transcripts. The CLN3 protein is known to exist in multiple isoforms, of which a few have a putative later start position - recently extensively reviewed in (Mirza et al, 2019) - and thus do not encompass the mutated c. $1 \mathrm{~A}>\mathrm{C}$ base pair as present in our patients. Possibly, these unaffected isoforms may provide the residual CLN3 protein production allowing a protracted neurological phenotype. An alternative hypothesis is that the mutated start codon itself is still translated. From in-vitro studies, non-AUG translation initiation has been shown to occur in a wide range of proteins, with some proteins even deriving solely from a non-AUG start codon. Since non-AUG translation initiation occurs at a much lower efficiency than 
the regular AUG translation initiation, this is probably one of the processes to regulate protein expression. Of the non-AUG start codons, the CUG alternative start codon - which corresponds to the $C .1 A>C$ start codon variant in our patients - has been suggested to provide the 'least inefficient' alternative start codon (Kearse and Wilusz, 2017). However, whether this tightly controlled regulatory process also applies to a variant in the start codon is speculative. In addition to transcriptional (regulatory) processes as described above, also, other - genetic, such as post-translational modifications, and probably nongenetic - modifying factors could impact residual CLN3 protein synthesis and function (Mirza et al, 2019). Regardless of the exact underlying mechanism, the protracted phenotype displayed by all three patients discussed in this report strongly indicates that the $\mathrm{C} .1 \mathrm{~A}>\mathrm{C}$ start codon variant in CLN3 allows some degree of residual protein synthesis.

Even with the start codon mutation being responsible for some degree of residual protein synthesis, it remains particularly puzzling why two of our patients displayed early onset of cognitive impairment, but thereafter a particularly slow decline/long plateau phase contrasting case 3 who finished regular high school but thereafter declined dramatically rapidly. Regarding the first two patients, particularly in light of (near) future therapeutic efficacy studies, prudence is warranted when classifying patients with an atypical genotype as having classical CLN3 disease, even if confirmed by an impaired IQ and motor score (as in case 1 ) as they might still later display a protracted phenotype. Regarding the third patient, insight in the chain of events that led the 'neuronal' balance to collapse so dramatically might also provide insight in neuronal deterioration in CLN3 disease in general.

In humans, previous work has shed light on the factors that determine whether a start codon variant will cause disease (Wolf et al, 2011). Our work suggests that future work should focus on the next step: the factors that determine whether a disease causing start codon variant completely abolishes protein synthesis or still allows some residual protein synthesis and the mechanism behind this. Insight herein will improve diagnosis, counseling, follow-up and possibly even therapy development analogous to the concept of nonsense codon read through (Lee et al, 2012; Welch et al, 2007). 


\section{ACKNOWLEDGMENTS}

We thank the parents/legal representatives of the patients for their consent to publish the case series. 


\section{REFERENCES}

Adams HR, Beck CA, Levy E et al (2010). Genotype does not predict severity of behavioural phenotype in juvenile neuronal ceroid lipofuscinosis (Batten disease). Dev Med Child Neurol. 52(7):637-43.

Chen CA, Bosch DG, Cho MT et al (2016). The expanding clinical phenotype of Bosch-BoonstraSchaaf optic atrophy syndrome: 20 new cases and possible genotype-phenotype correlations. Genet Med. 18(11):1143-1150.

Kearse MG, Wilusz JE (2017). Non-AUG translation: a new start for protein synthesis in eukaryotes. Genes Dev. 1;31(17):1717-1731.

Kousi M, Lehesjoki AE, Mole SE (2012). Update of the mutation spectrum and clinical correlations of over 360 mutations in eight genes that underlie the neuronal ceroid lipofuscinoses. Hum Mutat. 33(1):42-63.

Kozak M (1991). Structural features in eukaryotic mRNAs that modulate the initiation of translation. J. Biol. Chem. 266, 19867-19870.

Kuper WFE, van Alfen C, Rigterink RH, Fuchs SA, van Genderen MM, van Hasselt PM (2018). Timing of cognitive decline in CLN3 disease. J Inherit Metab Dis. 41(2):257-261.

Kuper WFE, van Alfen C, van Eck L et al (2019). Motor function impairment is an early sign of CLN3 disease (2019). Neurology. 16;93(3):e293-e297.

Lebrun AH, Moll-Khosrawi P, Pohl S et al (2011). Analysis of potential biomarkers and modifier genes affecting the clinical course of CLN3 disease. Mol Med. 17(11-12):1253-61.

Lee HL, Dougherty JP (2012). Pharmaceutical therapies to recode nonsense mutations in inherited diseases. Pharmacol. Ther. 136:227-266.

Munroe PB, Mitchison HM, O'Rawe AM et al (1997). Spectrum of mutations in the Batten disease gene, CLN3. Am J Hum Genet. 61(2):310-6.

Mirza M, Vainshtein A, DiRonza A et al (2019). The CLN3 gene and protein: What we know. Mol Genet Genomic Med. 2019 Sep 30:e859.

Nelson T, Pearce DA, Kovács AD (2017). Lack of specificity of antibodies raised against CLN3, the lysosomal/endosomal transmembrane protein mutated in juvenile Batten disease. Biosci Rep. 23;37(6).

Pittis MG, Ricci V, Guerci VI et al (2004). Acid sphingomyelinase: identification of nine novel mutations among Italian Niemann Pick type B patients and characterization of in vivo functional in-frame start codon. Hum Mutat. 24(2):186-7.

Sadhwani A, Sanjana NE, Willen JM et al (2018). Two Angelman families with unusually advanced neurodevelopment carry a start codon variant in the most highly expressed UBE3A isoform. Am J Med Genet A. 176(7):1641-1647.

Sargiannidou I, Kim GH, Kyriakoudi S, Eun BL, Kleopa KA (2015). A start codon CMT1X mutation associated with transient encephalomyelitis causes complete loss of $\mathrm{C} \times 32$. Neurogenetics. 16(3):193-200.

Welch EM, Barton ER, Zhuo J et al (2007). PTC124 targets genetic disorders caused by nonsense mutations. Nature. 447(7140):87-91.

Wolf A, Caliebe A, Thomas NS et al (2011). Single base-pair substitutions at the translation initiation sites of human genes as a cause of inherited disease. Hum Mutat. 32(10):1137-43.

Won JY, Kim D, Park SY et al (2019). Novel loss-of-function variants of TRAPPC2 manifesting X-linked spondyloepiphyseal dysplasia tarda: report of two cases. BMC Med Genet. 3;20(1):70. 



\section{CHAPTER A CASE OF UNEXPECTED ADULT-ONSET NEUROLOGIC DECLINE IN CLN3-ASSOCIATED RETINAL DEGENERATION}

Willemijn F.E. Kuper, Claudia van Alfen, Linda van Eck, Brigitte T.A. van den Broek, Albert Huisman, Maria M. van Genderen, Peter M. van Hasselt JAMA Ophthalmol. 2017 Dec 1;135(12):1451-1453

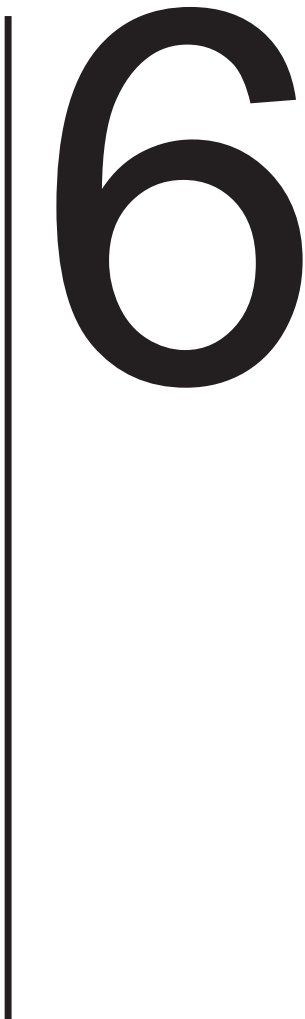




\section{INTRODUCTION}

Mutations in CLN3 (OMIM \#204200) lead to retinal degeneration in childhood, with additional development of cerebral neurodegeneration around the same age (classic CLN3) or up to adulthood (protracted CLN3). ${ }^{1}$ However, recent research claims that a subset of mutations in CLN3, notably the R405W missense mutation, give rise to isolated retinal degeneration. ${ }^{2,3}$ We describe a man in his early 30s homozygous for the R405W mutation initially diagnosed with isolated retinitis pigmentosa. In the past few years he started exhibiting neurologic decline.

\section{REPORT OF A CASE}

Retinitis pigmentosa was diagnosed in a man in his early 30s of Iraqi descent born to consanguineous parents after onset of slowly progressive vision loss, in particular night blindness and peripheral vision loss, from approximately age 12 years. The patient received mainstream education and subsequently worked as a factory worker and gardener. Genetic analysis performed during his mid-20s yielded a homozygous c.1213C $>\mathrm{T}$ (R405W) missense mutation in CLN3. This finding fit the isolated retinitis pigmentosa observed at that time. Light microscopy analysis revealed the presence of vacuolated lymphocytes, although less pronounced than usually seen in (classic) CLN3 disease. ${ }^{4}$ Over time, his best-corrected visual acuity deteriorated to $2.00 \log M A R$ (approximate Snellen equivalent, $3 / 300$ ) in his left eye, but, on recent examination, his bestcorrected visual acuity was $0.4 \log$ MAR (approximate Snellen equivalent, 20/50) in his right eye. Optical coherence tomography showed foveal sparing with photoreceptor loss from the perimacular area outward in his right eye and generalized photoreceptor loss, including macular atrophy, in his left eye (Figure).Despite the relatively intact central vision in his right eye, full-field electroretinography demonstrated undetectable rod and cone responses in both eyes.

Until his late 20s, the patient exhibited no extraocular symptoms, in particular, no neurologic problems. However, in the past 5 years, he gradually developed motor problems most prominent in his legs that were not explained by extensive analyses (magnetic resonance imaging of his lumbar spine, electromyogram, and muscle echography). On recent neurologic examination, he displayed a bipyramidal spastic paraparesis gait pattern, including lively 
reflexes in his legs, and low to normal reflexes and a cog-wheel rigidity in his arms. In addition, the patient increasingly experiences forgetfulness and wordfinding difficulties. Of note, the patient has a brother reportedly showing a similar combination of retinal and neurologic deterioration who has the same homozygous R405W missense CLN3 genotype.

FIGURE | FUNDOSCOPY AND OPTICAL COHERENCE TOMOGRAPHY (OCT) OF A PATIENT WITH CLN3-ASSOCIATED RETINAL DEGENERATION
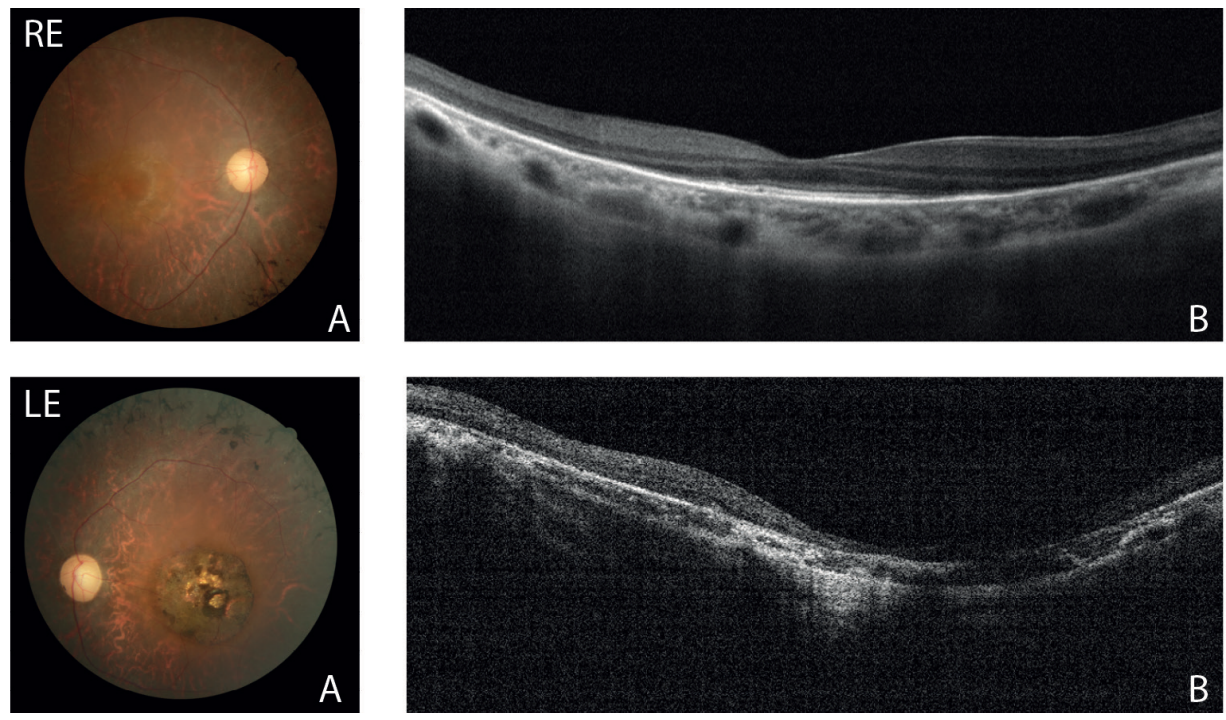

Patient's funduscopy and OCT at recent examination. A, Funduscopy shows narrow vessels, pale optic discs, and peripheral pigmentations in both eyes, a relatively intact macula in the right eye (RE), and macular pigment alterations and atrophy in the left eye (LE). B, Optical coherence tomography shows macular atrophy in the LE vs foveal sparing in the RE.

\section{DISCUSSION}

We describe a patient who initially presented with CLN3-associated isolated retinal degeneration but developed adult-onset neurologic decline, contrasting with the previous exclusive association of the R405W mutation with isolated vision loss. ${ }^{2,3}$ This observation indicates that some patients sharing this mutation-and perhaps other CLN3 mutations presently considered to be associated with isolated retinal degeneration-may be at an unknown risk 
for subsequent development of neurodegeneration. The marked phenotypic heterogeneity in patients sharing the same "mild" genotype suggests differences in the amount of residual protein activity between patients and implies a significant role for genetic and non-genetic modifying factors. ${ }^{5}$ It is known that the propensity of proteins to fold properly despite subtle errors is influenced by the availability of chaperones that aid the folding process. ${ }^{6}$ Future studies will have to delineate whether the onset of the ocular symptoms, which developed significantly earlier in our patient compared with other patients homozygous for the CLN3 R405W missense mutation, ${ }^{3}$ may serve as a predictor of the disease course. Until then, prudence may be warranted when counseling young patients with $C L N 3$ variants and apparently isolated retinal degeneration.

\section{ACKNOWLEDGMENTS}

We thank the patient for granting permission to publish this information. 


\section{REFERENCES}

1. Kousi M, Lehesjoki AE, Mole SE. Update of the mutation spectrum and clinical correlations of over 360 mutations in eight genes that underlie the neuronal ceroid lipofuscinoses. Hum Mutat. 2012;33(1):42-63.

2. Wang F, Wang H, Tuan HF, et al. Next generation sequencing-based molecular diagnosis of retinitis pigmentosa: identification of a novel genotype-phenotype correlation and clinical refinements. Hum Genet. 2014;133(3):331-345.

3. Ku CA, Hull S, Arno G, et al. Detailed clinical phenotype and molecular genetic findings in CLN3-associated isolated retinal degeneration. JAMA Ophthalmol. 2017;135(7):749-760.

4. Anderson G, Smith VV, Malone M, Sebire NJ. Blood film examination for vacuolated lymphocytes in the diagnosis of metabolic disorders; retrospective experience of more than 2,500 cases from a single centre. J Clin Pathol. 2005;58(12):1305-1310.

5. Lebrun AH, Moll-Khosrawi P, Pohl S, et al. Analysis of potential biomarkers and modifier genes affecting the clinical course of CLN3 disease. Mol Med. 2011;17(11-12):1253-1261.

6. McCabe ERB. Modifier genes: moving from pathogenesis to therapy [published online May 30, 2017]. Mol Genet Metab. doi:10.1016/j.ymgme.2017.05.018. 



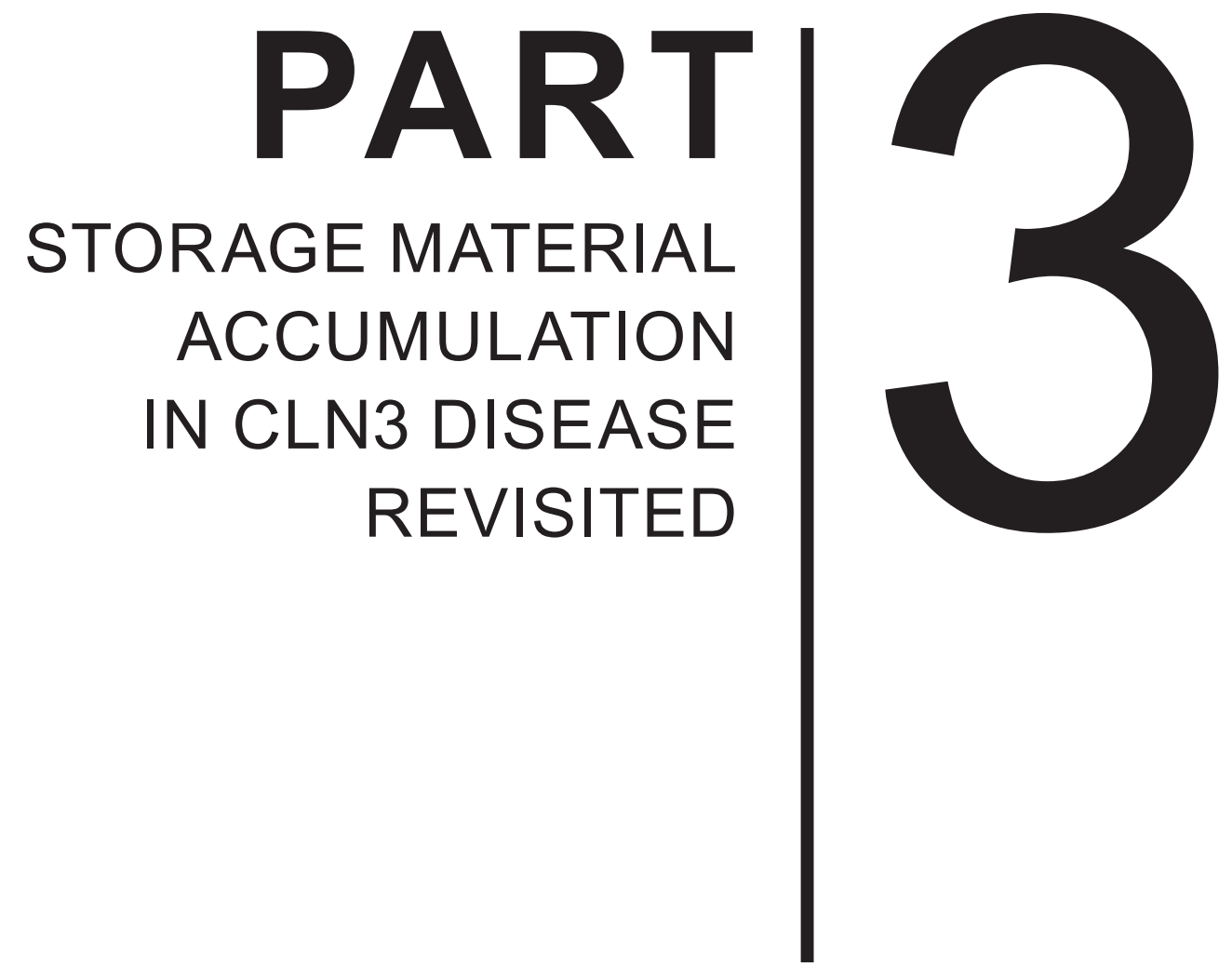





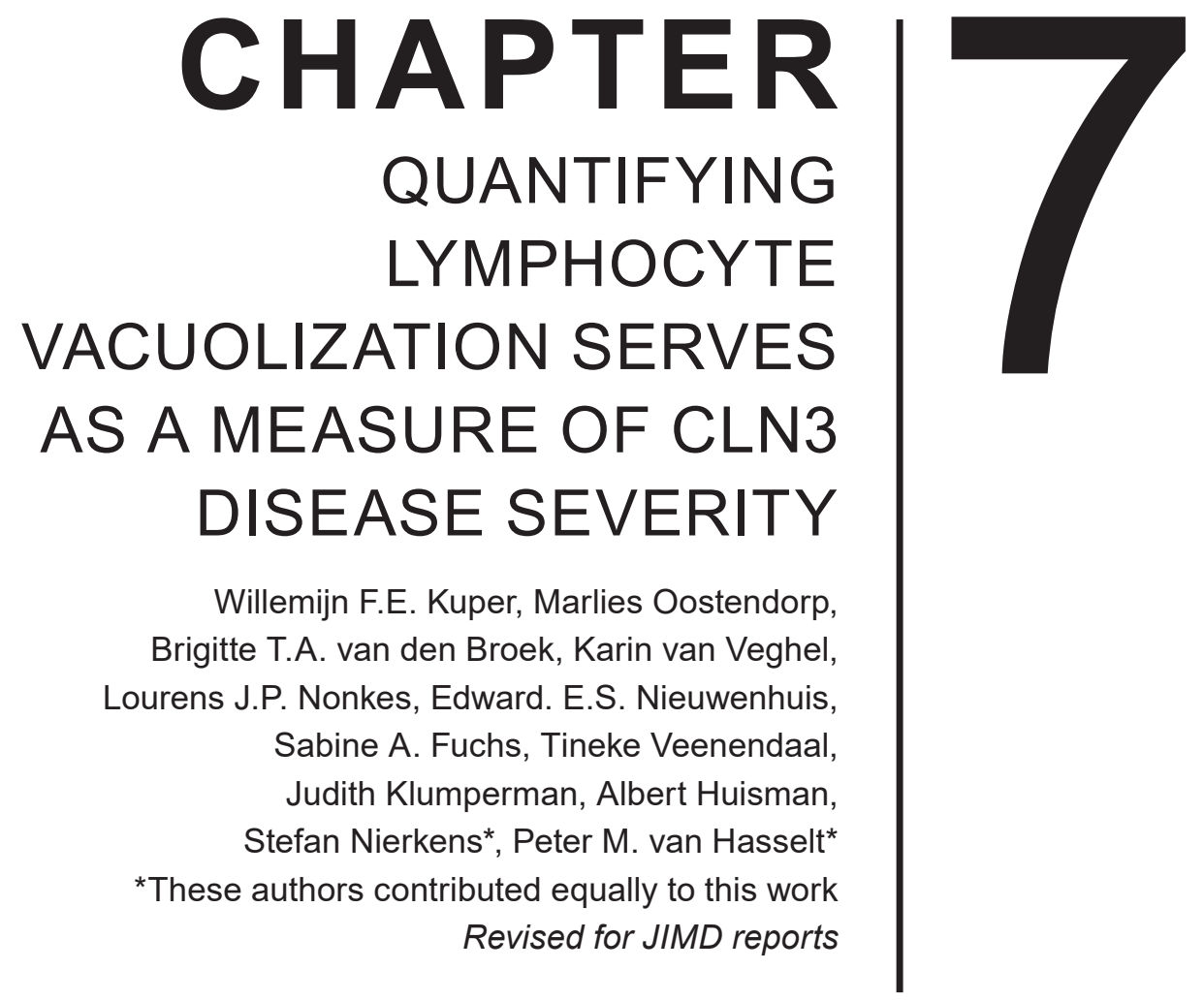




\section{ABSTRACT}

Background: Lymphocyte vacuolization is a hallmark of classical CLN3 disease, but differentiation with harmless vacuolization as observed during infections requires expertise, limiting its diagnostic use. We assessed if quantifying the degree of lymphocyte vacuolization could facilitate its use as a diagnostic marker for CLN3 disease and even as an index of disease severity.

Methods: Peripheral blood obtained from healthy controls and patients with CLN3 disease at diagnosis and during follow-up was used to assess lymphocyte vacuolization by 1) calculating the degree of vacuolization and by 2) quantifying expression of lysosomal associated membrane-1 (LAMP-1), using flow cytometry in lymphocyte subsets as well as a qualitative analysis using ImageStream and immuno-electron microscopy.

Results: Quantifying lymphocyte vacuolation not only allowed to differentiate controls from patients, but additionally allowed to differentiate between CLN3 patients according to disease severity $(p=0.0001)$. On immunofluorescence, classical CLN3 disease lymphocytes exhibited abundant vacuole-shaped LAMP-1 expression, suggesting its use as a proxy for lymphocyte vacuolization. Using flow cytometry in lymphocyte subsets, intracellular LAMP-1 expression was even more discriminative than vacuolization alone, additionally allowing to differentiate between storage and infection and to differentiate between CLN3 disease phenotypes even more in-depth. Intracellular LAMP-1 expression was highest in T-cells of patients with classical and protracted CLN3 disease while it was highest in B-cells at the mildest end of the disease spectrum.

Conclusion: Quantifying lymphocyte vacuolization allows to diagnose CLN3 disease and to classify disease severity. This may help interpretation of novel genetic variants and provide an individualized and quantitative readout for upcoming therapies.

Synopsis: Quantifying lymphocyte vacuolization - either manually or by determining flow cytometry derived LAMP-1 expression - serves as a measure of CLN3 disease severity.

Key Words: CLN3 disease; neuronal ceroid lipofuscinosis (NCL); lymphocyte vacuolization; lysosomal membrane associated protein-1 (LAMP-1); flow cytometry; ImageStream 


\section{INTRODUCTION}

CLN3 disease (OMIM \#204200) is the most common of the neuronal ceroid lipofuscinoses (NCLs), a genetically heterogeneous group of lysosomal storage disorders (LSDs) unified by the accumulation of autofluorescent storage material in all tissues with the retina and the brain as the predominantly affected tissues (Haltia and Goebel, 2013). CLN3 disease is caused by bi-allelic mutations in $C L N 3$, encoding a transmembrane protein involved in lysosomal homeostasis. While the retinal phenotype is relatively uniform throughout the disease spectrum, it is known that the severity of mutations in CLN3 determines the neurocognitive consequences of the disease (Kousi et al, 2012; Ku et al, 2017; Kuper et al, 2018; Kuper et al, 2019).

Abundant lymphocyte vacuolization in a school-aged child suffering from retinal dystrophy is pathognomonic for (classical) CLN3 disease (Anderson et al, 2005; Anderson et al, 2013; Aula et al, 1975). The degree of vacuolization may however be less abundant in non-classical forms of the disease (Kuper et al, 2017). Especially in these latter cases, differentiation from controls may be difficult, as some degree of lymphocyte vacuolization could also be due to a physiological response to a recent infection (Simon, 2003).

We hypothesized that quantifying lymphocyte vacuolization would provide an objective diagnostic marker that simultaneously allows to assess disease severity.

\section{METHODS}

\section{STUDY POPULATION}

Peripheral blood samples left over after routine analyses were obtained from 19 patients with genetically confirmed CLN3 disease and from five patients with other LSDs. The CLN3 disease cohort comprised the complete phenotypic spectrum, including 15 patients with classical CLN3 disease (deriving from two truncating mutations), one patient with delayed classical CLN3 disease, two patients with protracted CLN3 disease (both deriving from at least one relatively "mild" mutation), and one patient with CLN3-associated retinal degeneration (deriving from two relatively "mild" mutations) (Kousi et al, 2012; Ku et al, 2017; Kuper et al, 2017; Kuper et al, 2019) (Table 1). The patients with other LSDs comprised two patients with NCL subtypes in which lymphocyte 
vacuolization is absent (one patient with variant juvenile CLN1 disease, one patient with late infantile CLN5 disease) and three patients with other LSDs associated with lymphocyte vacuolization (two patients diagnosed with sialidosis type I, one patient diagnosed with alpha-mannosidosis) (Anderson et al, 2005).

A waiver for ethical review was granted by the Medical Ethical Research Committee of the University Medical Center Utrecht (18-024).

\section{CONTROLS}

First, control peripheral blood samples were obtained from six children in whom the diagnosis of CLN3 disease was ruled out: three patients whose retinal dystrophy was found to have a different cause (in the first patient bi-allelic mutations in $A B C A 4$ were found associated with Stargardt disease (Maugeri et al 2000); in the second patient bi-allelic mutations in CEP83 were found associated with a ciliopathy (Failler et al, 2014); in the third patient further genetic analyses were postponed) and three siblings of CLN3 disease patients who turned out to be heterozygous carriers of the common $1 \mathrm{~kb}$ deletion in CLN3.

Next, additional blood samples were collected from 22 healthy controls (10 used for peripheral blood smear analysis, 10 for flow cytometry, one for ImageStream and one for immuno-electron microscopy).

\section{AUTOMATED HEMATOLOGY ANALYSIS}

Complete blood count with standard 5-part leucocyte differentiation was performed using three commonly applied hematology analyzers that use optical light scatter (Abbot Cell-Dyn Sapphire and Sysmex XE-2100) and impedance technology (Abbot Cell-Dyn 1800) to differentiate between leucocyte subclasses (Chabot-Richards et al, 2015; Vis et al, 2016).

\section{PERIPHERAL BLOOD SMEAR ANALYSIS}

Peripheral blood smears were prepared using an automated slide maker stainer (Abbott Cell-Dyn SMS) and stained with May-Grünwald-Giemsa. Three experienced laboratory technicians independently counted the percentage of vacuolated lymphocytes in a total of 100 lymphocytes and the number of vacuoles per lymphocyte in a maximum of 20 vacuolated lymphocytes. 


\section{STAINING AND FLOW CYTOMETRY}

Lysated whole blood (EDTA anticoagulated) was incubated using directly labeled monoclonal antibodies against surface proteins (anti-CD56 BD Biosciences diluted 1:100; anti-CD20 BD Biosciences diluted 1:100; anti-CD3 BD Biosciences diluted 1:50; anti-CD4 Sony Biotechnology diluted 1:50; antiCD8 Biosciences diluted 1:50) and intracellular proteins (anti-LAMP-1 (CD107a) BD Biosciences diluted 1:25) and anti-perforin eBioscience diluted 1:50). An additional panel was constituted in which anti-LAMP-1 was replaced by antiLAMP-2 (CD107b BD Biosciences diluted 1:25). Directly following staining, cells were acquired on FACSCanto II and analyzed using FACS Diva Version 6.13 (BD Biosciences) or FlowJo version 7.6.5 software. Within the lymphocyte population, B-lymphocytes and T-lymphocytes were differentiated based respectively on $C D 20$ and $C D 3$ fluorescence. T-lymphocytes were further differentiated into CD4 and CD8 positive T cells. Non-B/T CD56 positive cells were categorized as NK cells. Gating for intracellular lysosomal accumulation was based on the NK cell population used as an internal positive control for both increased LAMP and perforin expression. To determine the degree of lysosomal accumulation, the percentage of affected cells - defined as the subset of cells positive for LAMP expression but negative for perforin expression - was measured for each lymphocyte subset.

IMAGING

IMMUNO-ELECTRON MICROSCOPY

Peripheral blood samples (sodium-heparin anticoagulated) collected from two classical CLN3 disease patients and one healthy control were mixed with equal amounts of PBS containing 2\% FBS and centrifuged at RT for 10 minutes at $600 \mathrm{~g}$ without brake. A concentrated leukocyte band (buffycoat) was collected and transferred to freshly prepared fixative containing $2 \%$ formaldehyde and $0.2 \%$ glutaraldehyde in $0.1 \mathrm{M}$ phosphate buffer $\mathrm{pH} 7.4$ for $10 \mathrm{~min}$, refreshed to continue fixation for $2 \mathrm{hr}$. Cells were stored in $1 \%$ formaldehyde at $4^{\circ} \mathrm{C}$ at least overnight, after which a second leucocyte concentration step was performed. Processing of cells for ultrathin cryosectioning and immuno-labeling according to the protein A-gold method was performed as described previously (Slot et al, 2007). In brief, fixed cells were washed with $0.05 \mathrm{M}$ glycin in PBS, resuspended and pelleted in $12 \%$ gelatin in PBS at $37^{\circ} \mathrm{C}$. The cell pellet was solidified on ice and cut into small blocks. For cryoprotection, blocks were infiltrated overnight with $2.3 \mathrm{M}$ sucrose at $4^{\circ} \mathrm{C}$, then mounted on aluminum pins and frozen in liquid nitrogen. A 1:1 mixture of $2.3 \mathrm{M}$ sucrose and $1.8 \%$ methylcellulose was used 
to pick up the ultrathin cryosections $(60 \mathrm{~nm})$. Grids were labeled with antiLAMP-1 (CD107A BD Biosciences) diluted 1:150 in PBS, 0,1\% BSA-C + 0.5 \% FSG. Followed by Protein A conjugated to $10 \mathrm{~nm}$ gold particles was homemade in UMC Utrecht (www.cellbiology-utrecht.nl/second-menu-cmc/products.html), diluted 1:50.

\section{IMAGESTREAM}

Peripheral blood samples (EDTA anticoagulated) collected from one classical CLN3 disease patient and one healthy control were incubated using directly labeled monoclonal antibodies against surface proteins (anti-CD3 BD Biosciences diluted 1:10; anti-CD4 Invitrogen diluted 1:50) and intracellular proteins (anti-LAMP-1 (CD107a) BD Biosciences diluted 1:25; anti-perforin eBioscience diluted 1:50). Expression of surface proteins and intracellular proteins in lymphocytes was measured by ImageStream (Amnis, Seattle, WA). A minimum of 10000 events were acquired for this analysis. Gating of lymphocytes was performed using the IDEAS software (v6.2). Subsequently all images of CD4 positive lymphocytes were extracted as 8-bit Tagged image file format (TIFF) images in a channel separated manner (LAMP-1, CD4) and imported in a customized script written in Python (Python v2.7) that was used to further process the images. First an Otsu's thresholding method based segmentation approach was used to obtain whole-cell masks of all lymphocytes. Hereby cell segmentation boundaries were based on the (surface) CD4 signal, as expressed in the CD4 channel. Using these segmentation masks the mean LAMP-1 expression for each individual cell was calculated. Additionally, using a 'sliding threshold' procedure the distribution of LAMP-1 expression in all lymphocytes was assessed. Thus, for each greyscale intensity level $(0-255,8$-bit) the percentage of cell surface that had a LAMP-1 intensity equal or above this intensity level was calculated. A total of 10492 CD4 positive lymphocytes were analyzed (healthy control: 2364 cells, classical CLN3 disease: 8128 cells)

\section{STATISTICAL ANALYSIS}

Patient characteristics were reported as frequencies and percentages for categorical variables and mean (SD) or median (range) for continuous variables. To analyze the effect of age as a proxy for disease progression on the percentage of vacuolated lymphocytes, and percentage of single positive LAMP-1 CD4, CD8, and CD20 cells, a linear mixed model was fitted with age and CLN3 phenotype as fixed effect. Based on the Akaike Information Criterion 
a random intercept was included per individual to account for individual variation in percentage of positive cells at baseline and a random slope (for age) for dependency across the repeated measurements within the same individual during follow-up. Final coefficients were estimated using restricted maximum likelihood. The model assumptions including normal distributed residuals, random effects, and homogeneity of variance were confirmed visually.

P-values $<0.05$ were considered significant. The $\mathrm{R}$ project for statistical computing version 3.4.1 was used for all mixed model analyses using the packages 'nlme' and 'ggplot2' (R Core Team, 2017).

\section{RESULTS}

\section{CLN3 DISEASE PATIENTS}

Between 2012 and 2019, we collected 67 peripheral blood samples from 19 patients with genetically confirmed CLN3 disease (Table 1 ).

\section{QUANTIFYING LYMPHOCYTE VACUOLIZATION}

Despite their grossly abnormal appearance (Figure 1), routine complete blood count analysis failed to detect vacuolated lymphocytes (Supplemental Figure $1 \mathrm{~A}, 1 \mathrm{~B}, 1 \mathrm{C})$. Thus, next, the percentage of vacuolated lymphocytes and the number of vacuoles per lymphocyte were quantified to determine their potential as a diagnostic test.

Lymphocyte vacuolization was rare with 1 to 3 percent of lymphocytes exhibiting some degree of vacuolization in both healthy controls and patients suffering from retinal dystrophy not caused by CLN3 disease, a relevant reference population. The few (1 to 5 per lymphocyte) vacuoles present were small and difficult to discern (Figure $1 \mathrm{~A}$ ). In contrast, vacuolization was abundant in patients with classical CLN3 disease (Figure 1A) with $11-69 \%$ of lymphocytes exhibiting vacuolization (median $30 \%$ ). The vacuoles in these patients were usually large, with the number per lymphocyte ranging from 6 to 18 (median 10). Of interest, patients with a milder disease severity exhibited fewer and smaller vacuoles (Figure $1 \mathrm{~A}$ ). The percentage of lymphocytes exhibiting vacuolization in patients with protracted CLN3 was 5 to $30 \%$ (median $8 \%$ ), with a median of 8 vacuoles per lymphocyte (ranged 3 to 12). In support of 
a gene dose effect, mixed model analysis which allows correction for repeated measurements, showed a statistically significant increase in vacuolization with increasing disease severity ( $p=0.0001$; Figure $1 B$ ). The degree of vacuolization was not associated with age, or longer follow up duration, arguing against vacuolization as a proxy for CLN3 disease progression ( $p=0.26$; Figure $1 B)$.

Of note, compared to the non-carrier healthy controls, CLN3 disease carriers appeared to display a slight increase in both the percentage of vacuolated lymphocytes (ranging from 4 to 9 ) and the number of vacuoles per lymphocyte (an average of 5 in all 3 individuals). In conclusion, the degree of lymphocyte vacuolization not only readily differentiates CLN3 disease patients from controls but also reflects disease severity.

\section{FIGURE 1A | LYMPHOCYTE VACUOLIZATION IN CLN3 DISEASE}

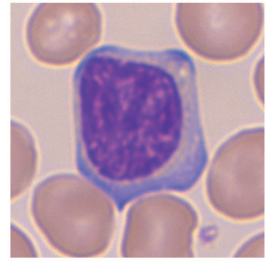

healthy control

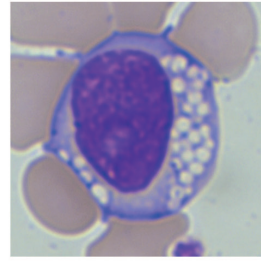

classical CLN3 disease

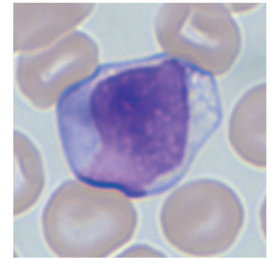

protracted CLN3 disease CLN3-as ret deg

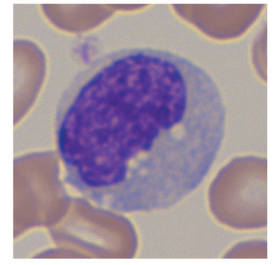

Light microcopy image of lymphocyte vacuolization in a healthy control (A) compared to classical CLN3 disease (B), protracted CLN3 disease (C), and CLN3 associated retinal degeneration (D). In CLN3 disease lymphocytes, the degree of vacuolization including the size of the vacuoles appeared to be related to disease severity. Abbreviations: CLN3-as ret deg $=$ CLN3-associated retinal degeneration 
FIGURE 1B | QUANTIFIED LYMPHOCYTE VACUOLIZATION IN CLN3 DISEASE

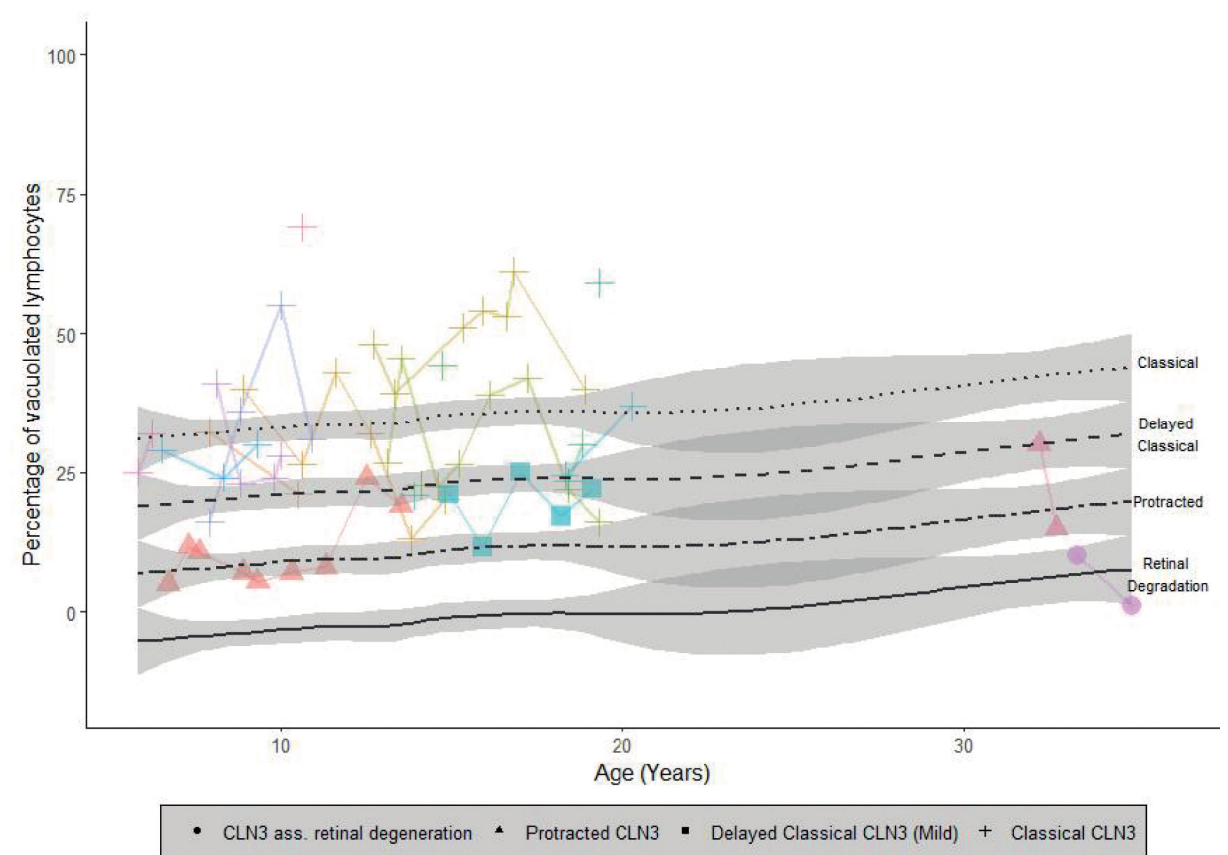

The percentage of vacuolated lymphocytes with a single positive LAMP-1 expression measured on flow cytometry. Connected lines show repeated measurements per patient. The linear mixed model estimates stable percentages of singe positive vacuolated lymphocytes per CLN3 disease severity type over time $(p=0.26)$ which is represented by the black lines.

Vacuoles in CLN3 disease are considered to be of lysosomal origin. We thus hypothesized that increased vacuolization is associated with a larger lysosomal compartment, hence an increased degree of lysosomal membrane protein expression. Indeed, using immunofluorescence on classical CLN3 disease lymphocytes, abundant LAMP-1 expression was observed in vacuole-shaped appearances, mirroring the vacuoles observed using light microscopy (Figure 1C). We next used flow cytometry to quantify LAMP-1 expression. 
FIGURE 1C | IMMUNOFLUORESCENCE DETECTS ABUNDANT LAMP-1

EXPRESSION IN CLN3 DISEASE LYMPHOCYTES

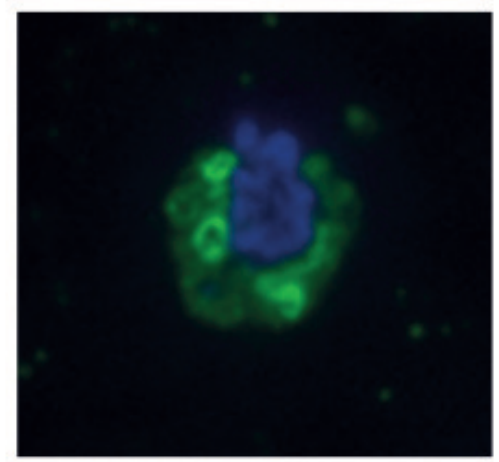

ImmunoFluorescence on $300 \mathrm{~nm}$ thick sections of lymphocytes of a classical CLN3 disease patient displaying abundant LAMP-1 expression (in green) appearing at the membranes of the vacuoles surrounding the nucleus (in blue).

Abbreviations: $L A M P-1=$ lysosomal associated membrane protein 1

\section{QUANTIFYING LAMP-1 EXPRESSION AS A PROXY FOR LYMPHOCYTE VACUOLIZATION}

Intracellular LAMP-1 expression was generally low in both controls and patients with CLN-subtypes (CLN1 and CLN5) without lymphocyte vacuolization. Any increases of LAMP-1 expression were only observed in the CD8 subset coinciding with increased perforin expression together indicating cytotoxic activation. In contrast, CLN3 disease lymphocytes exhibited an increased intracellular expression of both LAMP-1 (Figure 2) and LAMP-2 (data not shown), regardless of perforin expression. Mixed model analysis showed that LAMP-1 expression in both the CD4 and CD8 compartment correlated strongly with CLN3 disease severity ( $<<0.0001$ for both CD4 and CD8) (Figure $3 A$ and $B$ ). LAMP-1 expression did not change over time: expression of LAMP-1 was stable in all lymphocyte compartments $(p=0.53$ for $C D 4, p=0.11$ for $C D 8, p=0.14$ for the CD20 without the CLN3-associated retinal degeneration subtype). Enigmatically, LAMP-1 expression in the CD20 compartment was markedly and repeatedly-elevated in the CLN3-associated retinal degeneration patient (Figure 2 and Figure $3 \mathrm{C}$ ). Thus, quantifying LAMP-1 expression in lymphocytes using flow cytometry might even outperform microscopical assessment when classifying disease severity, especially at the mildest end of the disease spectrum. 


\section{FIGURE 2 | LYMPHOCYTE VACUOLIZATION AND LAMP-1 EXPRESSION CORRELATE WITH CLN3 DISEASE SEVERITY}
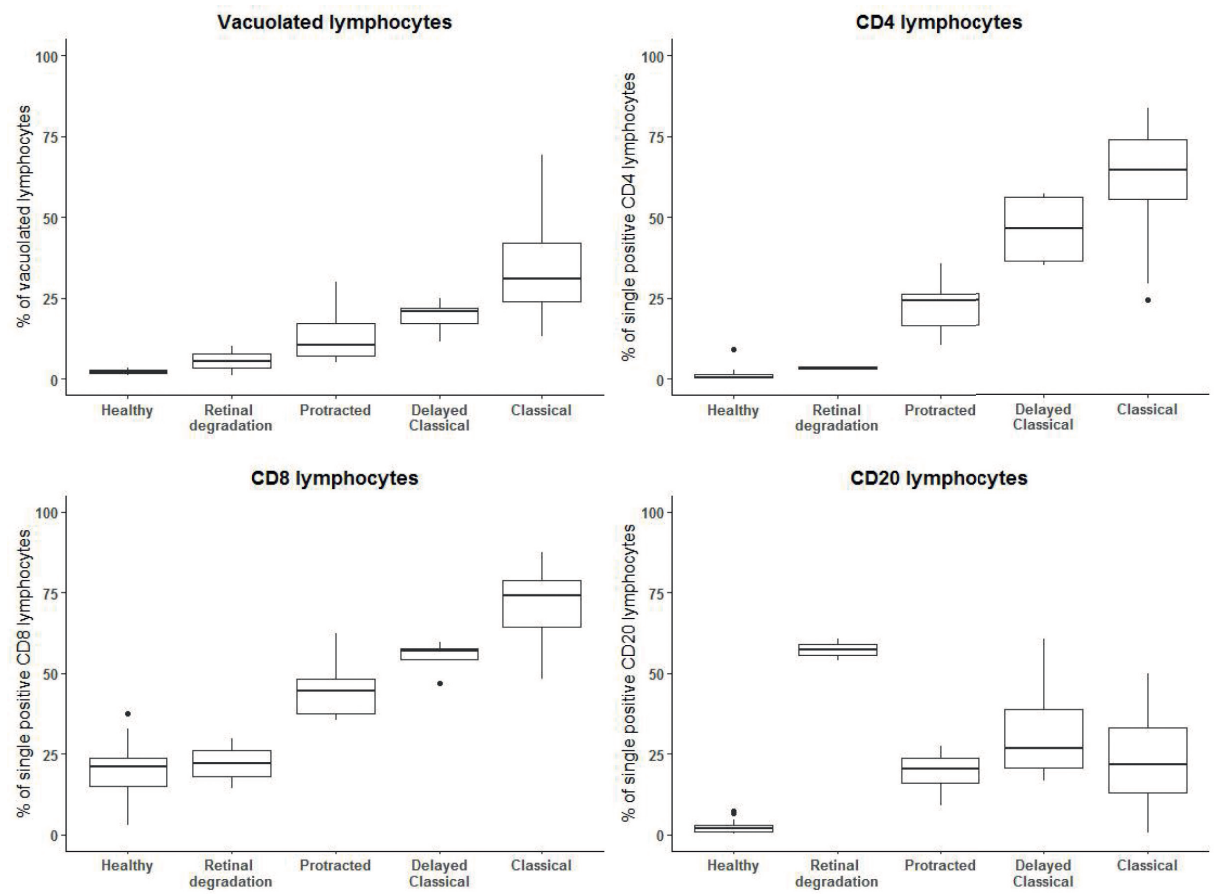

The percentage of vacuolated lymphocytes (left upper panel), percentage of LAMP-1 single positive CD4 (right upper panel), CD8 (left lower panel) and CD20 (right lower panel) lymphocytes split per phenotypic subtype shown in bar plots. In the percentage of vacuolated lymphocytes, and single positive CD4 and CD8 lymphocytes a clear correlation with disease severity is seen. In the CD20 subset, a particularly high degree of LAMP-1 expression is seen in the most mild CLN3 disease phenotype: CLN3-associated retinal degeneration.

Abbreviations: $L A M P-1=$ lysosomal associated membrane protein 1 
FIGURE 3 | FLOW CYTOMETRY ANALYSES OF LAMP-1 EXPRESSION IN CLN3 DISEASE LYMPHOCYTES

A

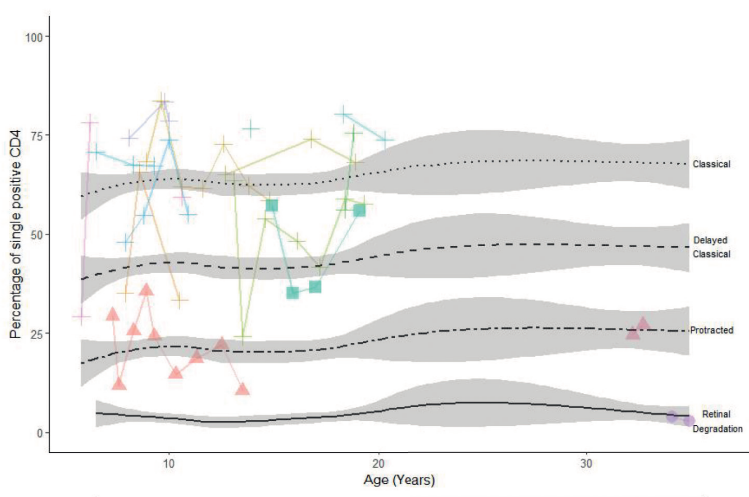

- CLN3 ass retinal degeneration - Protracted CLN3 - Delayed Classical CLN3 (Mid) + Classical CLN3

B

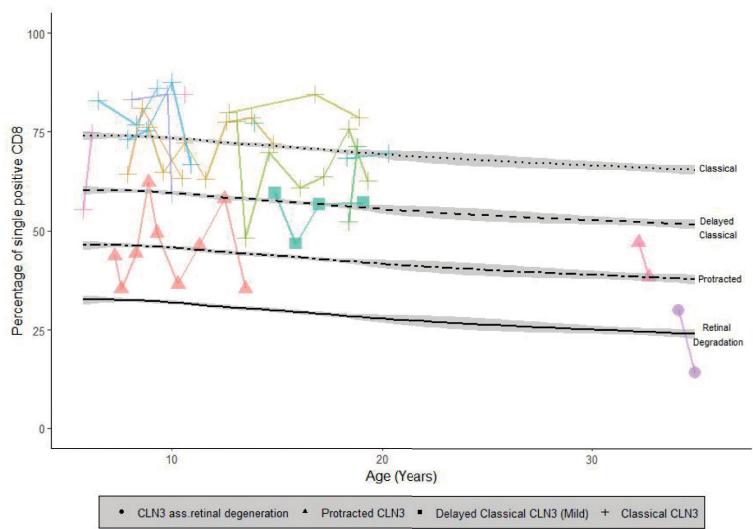

C

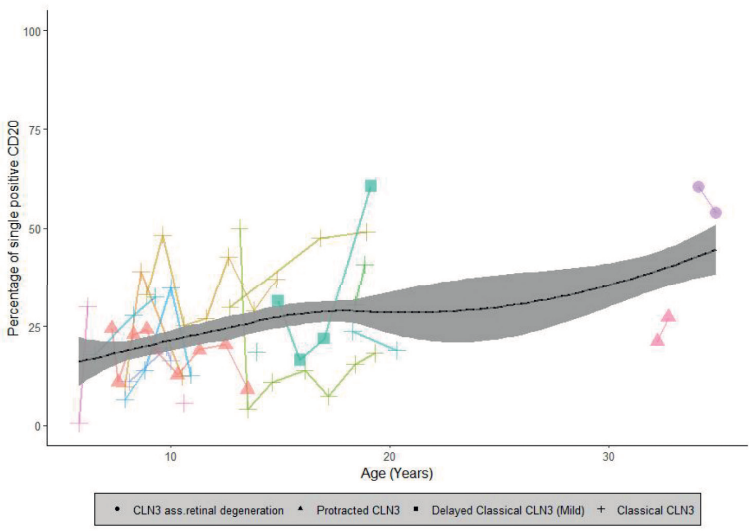


The percentage of lymphocytes with a single positive LAMP-1 expression measured on flow cytometry. A: CD4; B: CD8; C: CD20. Connected lines show repeated measurements per patient. The linear mixed model estimates stable percentages of singe positive lymphocytes per CLN3 disease severity type over time which is represented by the black lines.

Abbreviations: $L A M P-1=$ lysosomal associated membrane protein 1

Contrary to our expectations, we noted that increased LAMP-1 expression was observed in all lymphocytes of patients with CLN3 disease, thus including the many lymphocytes which did not exhibit vacuolization. This observation implies that, rather than a consequence of vacuolization per se, the increased LAMP-1 expression reflects a more global enlargement of the lysosomal compartment evoked by CLN3 dysfunction. Subsequent ImageStream analysis confirmed that the increased LAMP-1 expression was found in all cells and confirmed that it was not restricted to vacuoles, but rather was distributed throughout the cell (Figure 4). This notion was further supported using immuno-electron microscopy, which showed increased LAMP-1 expression not only around vacuolated lysosomes but also around non-vacuolated lysosomes and (non-vacuolated) late endosomes (Figure 5), indicative of an overall enlarged lysosomal compartment. 
FIGURE 4 | IMAGESTREAM ANALYSIS TO VISUALIZE LAMP-1 DISTRIBUTION WITHIN CLN3 DISEASE LYMPHOCYTES

A

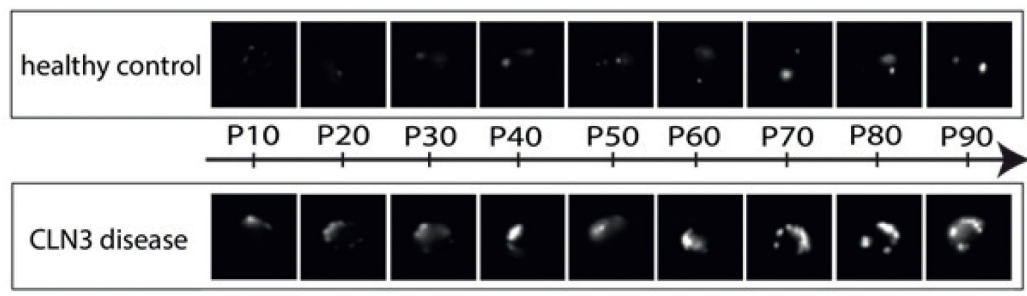

B

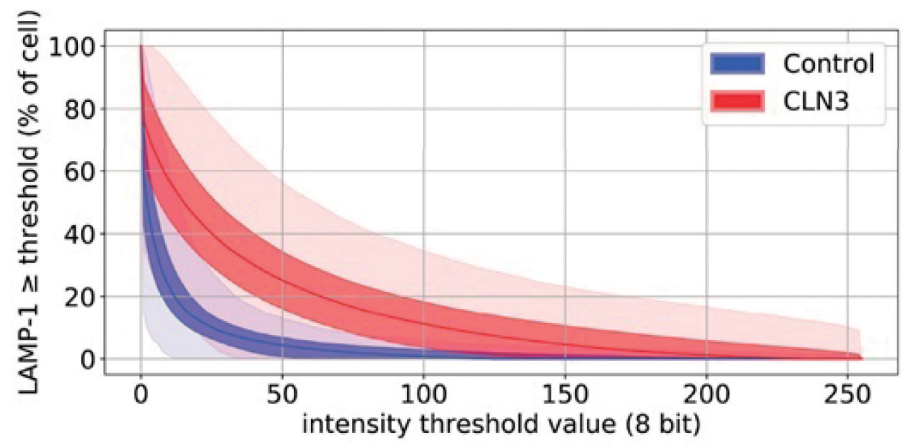

ImageStream analysis of classical CLN3 disease lymphocytes compared to healthy control lymphocytes confirmed increased expression of LAMP-1 in CLN3 disease lymphocytes distributed throughout a major area of the cell. A) Examples of LAMP-1 expression in CD4 positive lymphocytes. The mean LAMP-1 expression / cell was calculated for all lymphocytes per condition (healthy versus CLN3). Shown are representative examples of mean LAMP-1 expression / cell at the 10th up to 90th percentile of the distributions. B) Median LAMP-1 intensity expressed as \% of cell above/equal to a specified intensity threshold value (x-axis), CLN3 disease versus healthy control. Shaded ranges: dark band represents interquartile range (25th - 75th percentile), light band 2.5th - 97.5th percentile range.

Abbreviations: $L A M P-1=$ lysosomal associated membrane protein 1

We suspected that the expression of LAMP-1 in lymphocytes could also help detect other disorders known to exhibit lymphocyte vacuolization (Anderson et al, 2005). Indeed, an exploratory analysis in two sialidosis patients and one alfa mannosidosis patient confirmed that increased LAMP-1 expression paralleled increased lymphocyte vacuolization upon light microscopy. Interestingly, while in alfa mannosidosis, an increased expression of LAMP-1 was seen mainly in the CD4 and CD8 (T-cell) compartment similar to classic- 
protracted CLN3 disease (Supplemental Figure 2), increased LAMP-1 expression was especially profound in the CD20 (B-cell) compartment of patients with sialidosis (Supplemental Figure 3).

These results indicate that LAMP-1 expression may be used to aid diagnosis of diseases associated with lymphocyte vacuolization and furthermore implies that its distribution within lymphocyte subsets may represent a disease specific 'signature'. 
FIGURE 5 | IMMUNO-ELECTRON MICROCOPY IMAGING OF LAMP-1

EXPRESSION AND DISTRIBUTION IN CLN3 DISEASE LYMPHOCYTES
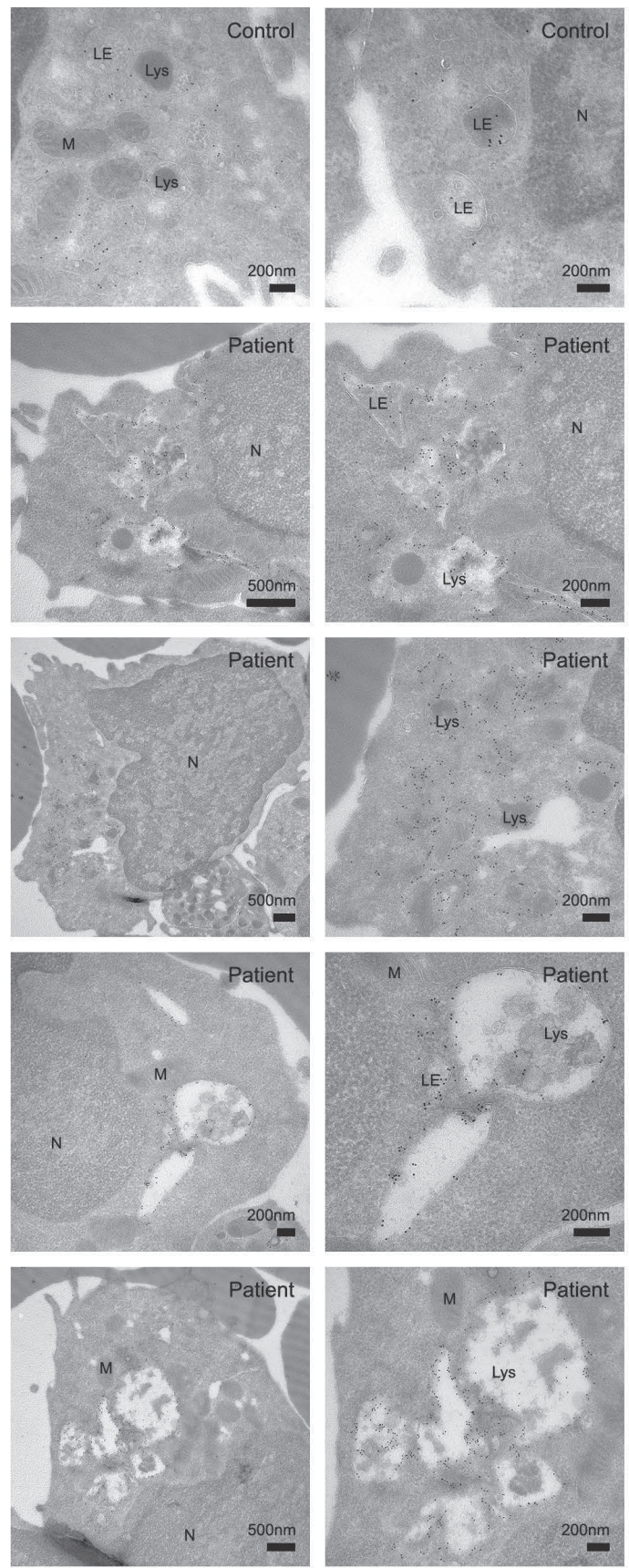
Immuno-electron microscopy of ultrathin cryosections labeled with LAMP-1 - $10 \mathrm{~nm}$ protein A gold particles. Compared to a healthy control, lymphocytes from a classical CLN3 disease patient show an increased LAMP-1 expression at the membranes of vacuolated lysosomes, but also around non-vacuolated lysosomes and late endosomes. Abbreviations: $N=$ nucleus; $M=$ mitochondrion; $L E=$ late endosome; $L y s=$ lysosome

\section{DISCUSSION}

In this study, we demonstrate that quantifying lymphocyte vacuolization both allows to rapidly diagnose CLN3 disease and to classify disease severity.

Our findings illustrate the potential added value of the use of a flow cytometry assay when assessing a metabolic disease. Not only does it allow differentiate patients from controls, but in addition it allows to delineate differences in storage material accumulation between patients and controls and even between different cell types. We unveiled that in classical and protracted CLN3 disease, LAMP-1 expression seems most pronounced in T-lymphocytes. Putatively, the ability of T lymphocytes - mainly the CD8 subset but also the CD4 subset - to form cytotoxic granules to battle infection also makes this compartment more prone to produce vacuoles in classical-protracted CLN3 disease. Enigmatically, at the mildest end of the disease spectrum, in CLN3-associated retinal degeneration, increased LAMP-1 expression was confined to B-lymphocytes. This predominant B-cell involvement was also observed in the two sialidosis type I patients we measured. Sialidosis type I, a different LSD, is characterized by progressive vision loss due to a (macular cherry red spot type of) retinal dystrophy in addition to gait impairment due to progressive myoclonus and ataxia resembling a spastic paraparesis (Schene et al, 2016). Interestingly, while spastic paraparesis is not a symptom of (classical-protracted) CLN3 disease, it is present in the CLN3-associated retinal degeneration patient included in this study (Kuper et al, 2017). It is tempting to speculate that this clinical overlap reflects a shared pathophysiological mechanism, but future research is needed to elaborate on this. Currently, we have included the LAMP-1 expression assay in the diagnostic process in our hospital and we foresee that this assay as well as other flow cytometry based assays will play a larger role in the diagnostic process of metabolic diseases, and in disease monitoring.

Contrary to a previous report, we did not find evidence that lymphocyte vacuolization in CLN3 disease increases in parallel with disease progression (Kimura et al, 1988). In our study, any increase with age disappeared when patients were grouped according to the severity of the underlying genotype. 
Likely, the mild increase observed in the Kimura study - involving a relatively small cohort with a short follow-up period - was either due to inter-patient differences in the degree of storage material accumulation related to genotypic severity not yet known at that time (Kimura et al, 1988; The International Batten Disease consortium, 1995) or due to intra-patient differences in the degree of storage material accumulation. This variation in storage material levels within a patient may be partly due to inter-observer differences in quantifying lymphocyte vacuolization, but, since some degree of variation was also present in our study in the automated measurement of LAMP-1 expression, it may also be the result of an active balance between build-up and removal of storage material dependent on residual CLN3 protein activity.

Biomarker identification in humans has some limitations, particularly with rare disorders. Limitations include small patient sample sizes and difficulties in obtaining tissue of interest, aggravated by the strict ethical requirements for using body material from children and/or incapacitated individuals. One approach to overcome this could be to first identify biomarkers in animal models, such as mice. Indeed, one biomarker identification study in mice reported a (minor) increase in lymphocyte vacuolization with age, in addition to several other possible hematological biomarkers for CLN3 disease (Staropoli et al, 2012). However, since hematological parameters show major variabilities between and even within different mouse inbred strains (Timm et al, 2018) this has - at least for CLN3 disease - not yet led to the identification of clinically relevant biomarkers. Although we acknowledge the use of mice models for fundamental pathophysiology research, we advise the use of human material whenever available - for instance material left-over from routine analyses as we did in this study - to identify biomarkers relevant for the patient.

In conclusion, quantifying lymphocyte vacuolization allows to diagnose CLN3 disease and to classify disease severity. This may help interpretation of novel genetic variants and may provide an individualized \& quantitative readout for upcoming therapies (Parisi et al, 2018). 


\section{ACKNOWLEDGMENTS}

We thank Evertine Heek, Amelia Lacna and Jeroen van Velzen for their technical support. 


\section{REFERENCES}

Anderson G, Smith VV, Malone M, Sebire NJ. Blood film examination for vacuolated lymphocytes in the diagnosis of metabolic disorders; retrospective experience of more than 2,500 cases from a single centre. J Clin Pathol. 2005 Dec;58(12):1305-10.

Anderson GW, Goebel HH, Simonati A. Human pathology in NCL. Biochim Biophys Acta. 2013;1832(11):1807-26.

Aula P, Rapola J, Andersson LC. Distribution of cytoplasmic vacuoles in blood T and B lymphocytes in two lysosomal disorders. Virchows Arch B Cell Pathol. 1975 Sep 11;18(4):263-71.

Chabot-Richards DS, George TI. White blood cell counts: reference methodology. Clin Lab Med. 2015 Mar;35(1):11-24.

Failler M, Gee HY, Krug P et al. Mutations of CEP83 cause infantile nephronophthisis and intellectual disability. Am J Hum Genet. 2014 Jun 5;94(6):905-14.

Haltia M, Goebel HH. The neuronal ceroid lipofuscinoses: a historical introduction. Biochim Biophys Acta. 2013 Nov;1832(11):1795-800.

Kimura S, Goebel HH. Light and electron microscopic study of juvenile neuronal ceroidlipofuscinosis lymphocytes. Pediatr Neurol. 1988 May-Jun;4(3):148-52.

Kousi M, Lehesjoki AE, Mole SE. Update of the mutation spectrum and clinical correlations of over 360 mutations in eight genes that underlie the neuronal ceroid lipofuscinoses. Hum Mutat. 2012;33(1):42-63.

Ku CA, Hull SH, Arno G et al. Detailed clinical phenotype and molecular genetic findings in CLN3-associated isolated retinal degeneration. JAMA Ophthalmol. 2017;135(7):749-760.

Kuper WFE, van Alfen C, Rigterink RH, Fuchs SA, van Genderen MM, van Hasselt PM. Timing of cognitive decline in CLN3 disease. J Inherit Metab Dis. 2018 Mar;41(2):257-261.

Kuper WFE, van Alfen C, van Eck L et al. Motor function impairment is an early sign of CLN3 disease. Neurology. 2019 Jul 16;93(3):e293-e297.

Kuper WFE, van Alfen C, van Eck L et al. A case of unexpected adult-onset neurologic decline in CLN3-associated retinal degeneration. JAMA Ophthalmol. 2017;135(12):1451-1453.

Kuper WFE, van Alfen C, van Eck $L$ et al. The $c .1 \mathrm{~A}>\mathrm{C}$ start codon mutation in CLN3 is associated with a protracted disease course. In press (JIMD reports 2020).

Maugeri A, Klevering BJ, Rohrschneider $\mathrm{K}$ et al. Mutations in the ABCA4 (ABCR) gene are the major cause of autosomal recessive cone-rod dystrophy. Am J Hum Genet. 2000 Oct;67(4):960-6.

Parisi D, Musumeci O, Mondello S et al. Vacuolated PAS-positive lymphocytes on blood smear: an easy screening tool and a possible biomarker for monitoring therapeutic responses in late onset pompe disease (LOPD). Front Neurol. 2018 Oct 22;9:880.

R Core Team (2017). R: A language and environment for statistical computing. R Foundation for Statistical Computing, Vienna, Austria. URL: https://www.R-project.org/.

Schene IF, Kalinina Ayuso V, de Sain-van der Velden M et al. Pitfalls in diagnosing neuraminidase deficiency: psychosomatics and normal sialic acid excretion. JIMD Rep. 2016;25:9-13.

Simon MW. The atypical lymphocyte. International Pediatrics. 2003; 18(1):20-22.

Slot JW, Geuze HJ. Cryosectioning and immunolabeling. Nature Protocols. 2007 Vol. 2 No 10: 2480-2491.

Staropoli JF, Haliw L, Biswas S et al 2012. Large-scale phenotyping of an accurate genetic mouse model of JNCL identifies novel early pathology outside the central nervous system. PLoS One. 2012;7(6):e38310. 
The International Batten Disease Consortium. Isolation of a novel gene underlying Batten disease, CLN3. Cell. 1995 Sep 22;82(6):949-57.

Timm D, Cain JT, Geraets RD et al. Searching for novel biomarkers using a mouse model of CLN3-Batten disease. PLoS One. 2018 Aug 7;13(8):e0201470. doi: 10.1371/journal. pone.0201470. eCollection 2018.

Vis JY, Huisman A. Verification and quality control of routine hematology analyzers. Int J Lab Hematol. 2016 May;38 Suppl 1:100-9. 
TABLE 1 | OVERVIEW OF CLN3 DISEASE PATIENTS AND SAMPLES

\begin{tabular}{|c|c|c|c|}
\hline $\begin{array}{l}\text { No of } \\
\text { patients }\end{array}$ & CLN3 genotype & Phenotype & $\begin{array}{l}\text { No of peripheral } \\
\text { blood samples }\end{array}$ \\
\hline \multicolumn{4}{|c|}{ Classical CLN3 disease } \\
\hline 10 & $\begin{array}{l}1 \text { kb deletion in } \\
\text { homozygous form }\end{array}$ & $\begin{array}{l}\text { Childhood onset retinal } \\
\text { dystrophy } \\
\text { Childhood onset } \\
\text { neurodegeneration }\end{array}$ & 29 \\
\hline 1 & $\begin{array}{l}\text { Deletion of exon 9-15 } \\
\text { in homozygous form }\end{array}$ & $\begin{array}{l}\text { Childhood onset retinal } \\
\text { dystrophy } \\
\text { Childhood onset } \\
\text { neurodegeneration }\end{array}$ & 7 \\
\hline 1 & $\begin{array}{l}\text { c. } 1054 C>T \text { nonsense } \\
\text { mutation in } \\
\text { homozygous form }\end{array}$ & $\begin{array}{l}\text { Childhood onset retinal } \\
\text { dystrophy } \\
\text { Childhood onset } \\
\text { neurodegeneration }\end{array}$ & 8 \\
\hline 2 & $\begin{array}{l}1 \mathrm{~kb} \text { deletion and } \\
\text { delG561 in exon } 6\end{array}$ & $\begin{array}{l}\text { Childhood onset retinal } \\
\text { dystrophy } \\
\text { Childhood onset } \\
\text { neurodegeneration }\end{array}$ & 2 \\
\hline 1 & $\begin{array}{l}1 \mathrm{~kb} \text { deletion and } \\
\text { c. } 379 \text { delC }\end{array}$ & $\begin{array}{l}\text { Childhood onset retinal } \\
\text { dystrophy } \\
\text { Childhood onset } \\
\text { neurodegeneration }\end{array}$ & 1 \\
\hline \multicolumn{4}{|c|}{ Delayed classical CLN3 disease } \\
\hline 1 & $\begin{array}{l}1 \mathrm{~kb} \text { deletion and } \\
\text { c. } 1000 \mathrm{C}>\mathrm{T} \text { missense } \\
\text { mutation }\end{array}$ & $\begin{array}{l}\text { Childhood onset retinal } \\
\text { dystrophy } \\
\text { Adolescence onset } \\
\text { neurodegeneration }\end{array}$ & 5 \\
\hline \multicolumn{4}{|c|}{ Protracted CLN3 disease } \\
\hline 1 & $\begin{array}{l}\text { c. } 139 \mathrm{~T}>\mathrm{C} \text { missense } \\
\text { mutation and } \\
\text { c. } 1000 \mathrm{C}>\mathrm{T} \text { missense } \\
\text { mutation }\end{array}$ & $\begin{array}{l}\text { Childhood onset retinal } \\
\text { dystrophy } \\
\text { Late adolescence } \\
\text {-adult onset } \\
\text { neurodegeneration }\end{array}$ & 2 \\
\hline 1 & $\begin{array}{l}1 \mathrm{~kb} \text { deletion and } \\
\text { c. } 1 \mathrm{~A}>\mathrm{C} \text { missense } \\
\text { mutation }\end{array}$ & $\begin{array}{l}\text { Discussed in Kuper et } \\
\text { al, } 2020\end{array}$ & 10 \\
\hline
\end{tabular}


TABLE 1 | CONTINUED

\begin{tabular}{llll}
\hline $\begin{array}{l}\text { No of } \\
\text { patients }\end{array}$ & CLN3 genotype & Phenotype & $\begin{array}{l}\text { No of peripheral } \\
\text { blood samples }\end{array}$ \\
\hline CLN3-associated retinal degeneration & \\
\hline 1 & $\begin{array}{l}\text { C.1213C>T missense } \\
\text { mutation in } \\
\text { homozygous form }\end{array}$ & $\begin{array}{l}\text { Discussed in Kuper et } \\
\text { al, 2017 }\end{array}$ & 3 \\
& & \\
\hline
\end{tabular}


SUPPLEMENTAL FIGURE 1A | AUTOMATED HEMATOLOGICAL ANALYZERS DO NOT DETECT ABUNDANT LYMPHOCYTE VACUOLATION IN CLASSICAL CLN3 DISEASE
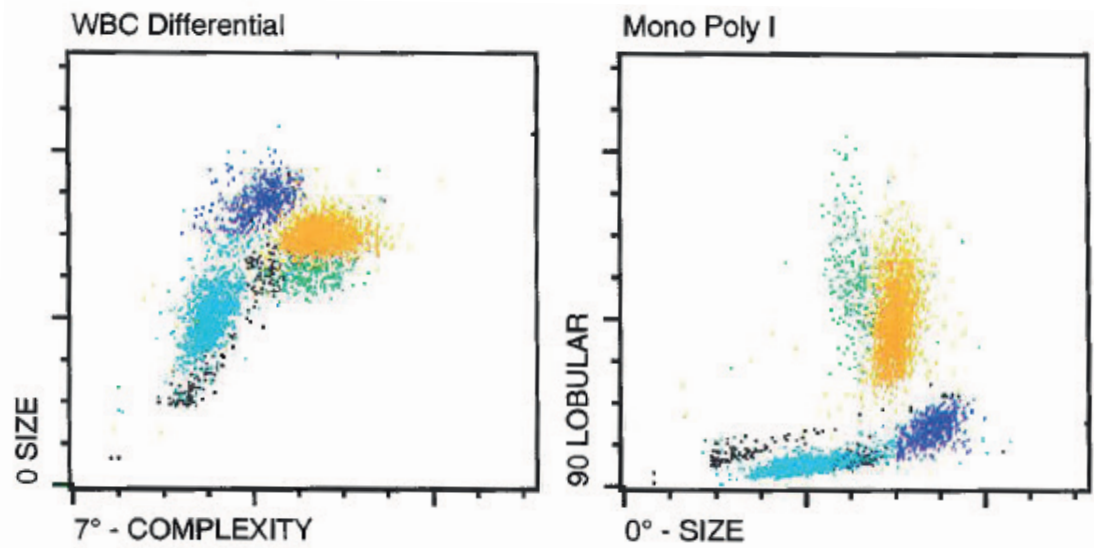

Optical PLT

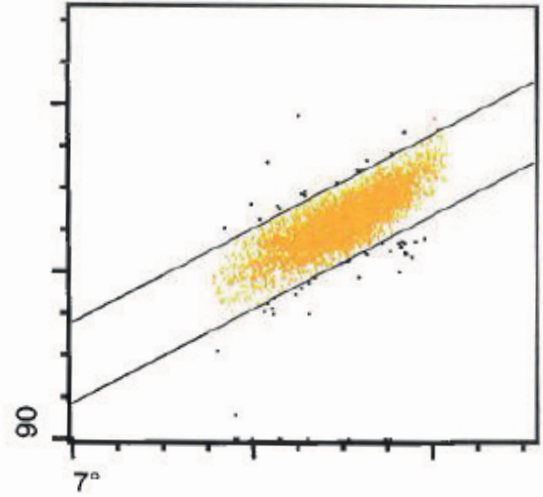

Impedance RBC

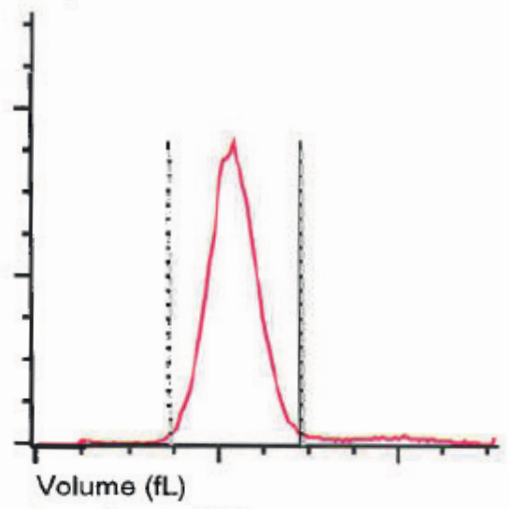

Optical PLT

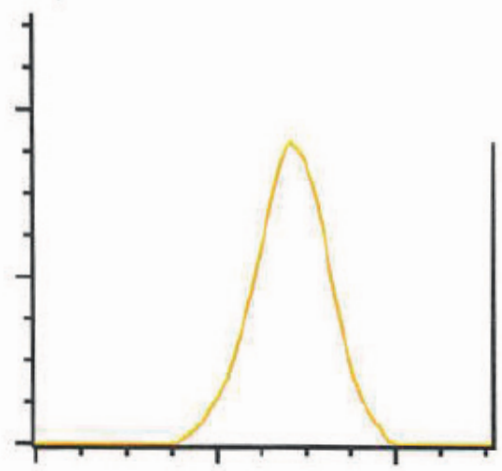

Impedance PLT

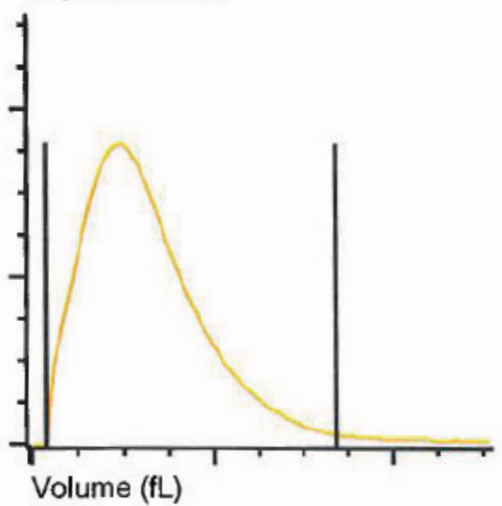


SUPPLEMENTAL FIGURE 1B | AUTOMATED HEMATOLOGICAL ANALYZERS DO NOT DETECT ABUNDANT LYMPHOCYTE VACUOLATION IN CLASSICAL CLN3 DISEASE

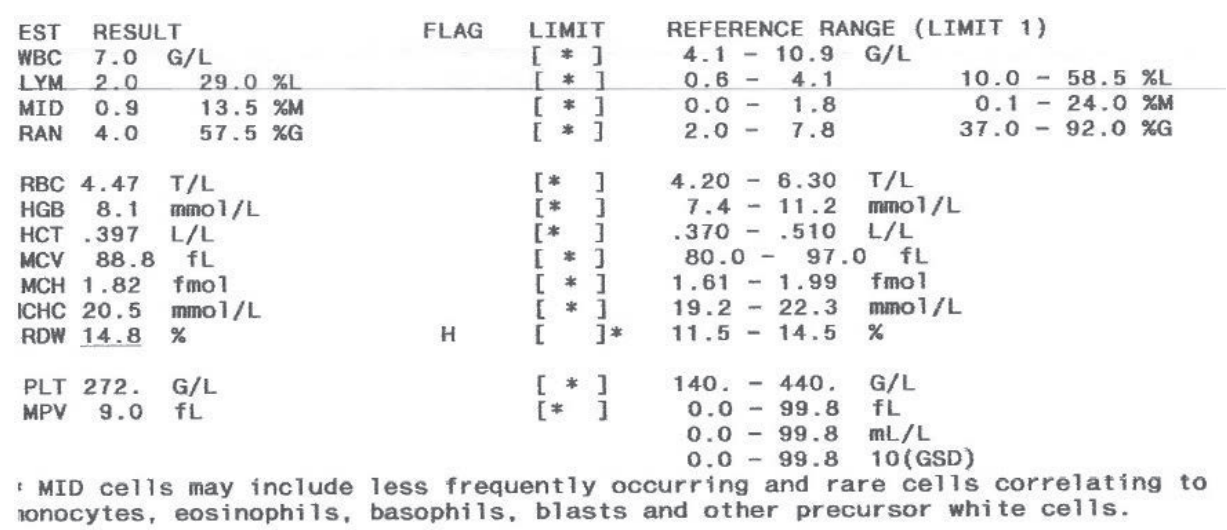

IBC

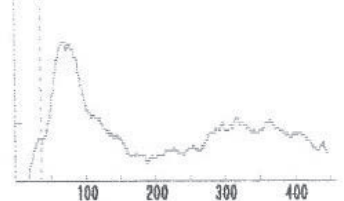

$4.55 \mathrm{~s}$

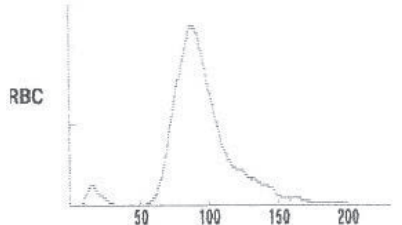

$6.69 \mathrm{~s}$

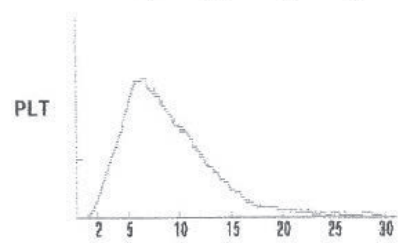


SUPPLEMENTAL FIGURE 1C | AUTOMATED HEMATOLOGICAL ANALYZERS DO NOT DETECT ABUNDANT LYMPHOCYTE VACUOLATION IN CLASSICAL CLN3 DISEASE

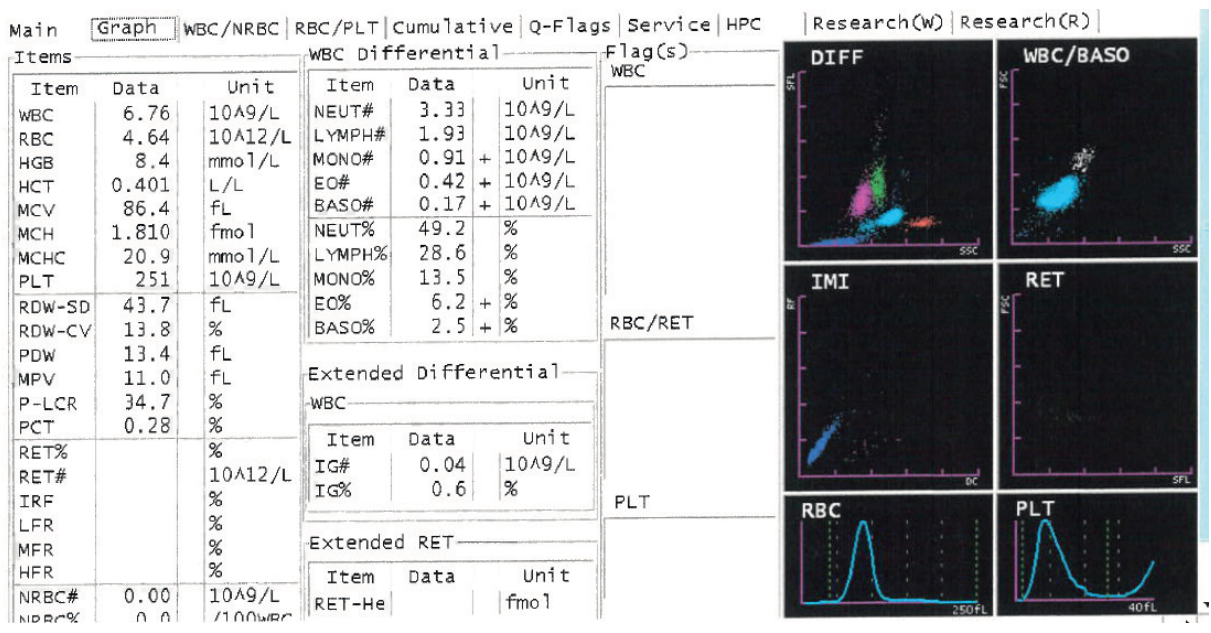


SUPPLEMENTAL FIGURE 2 | LAMP-1 EXPRESSION IN ALFA MANNOSIDOSIS COMPARED TO CLASSICAL CLN3 DISEASE

\section{A | CLASSICAL CLN3 DISEASE}
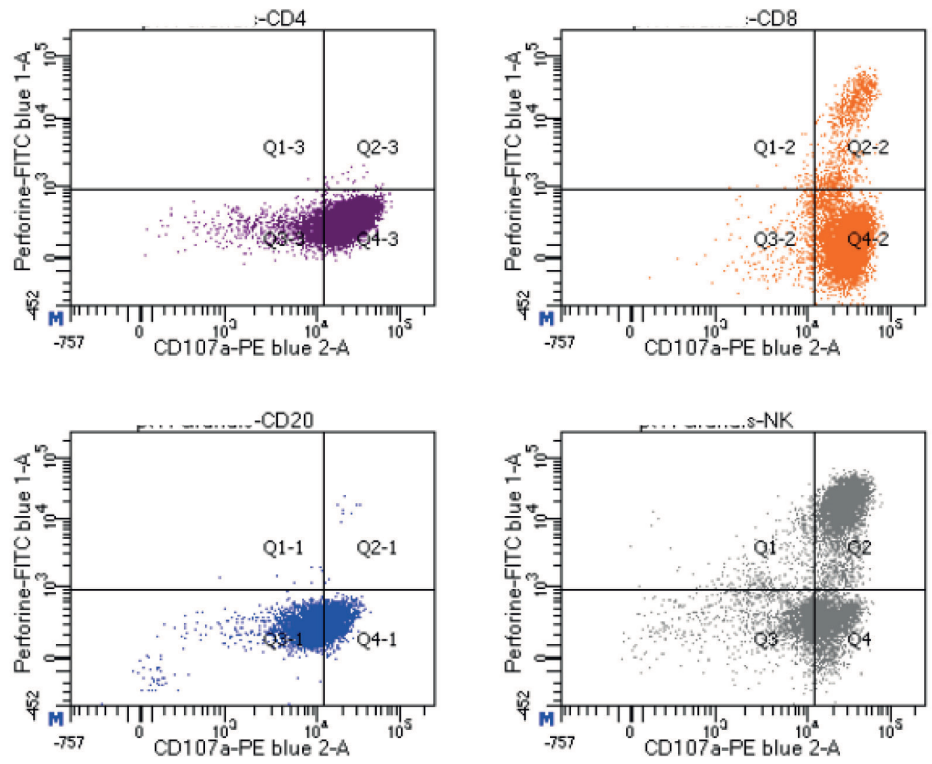

\section{B | ALFA MANNOSIDOSIS}
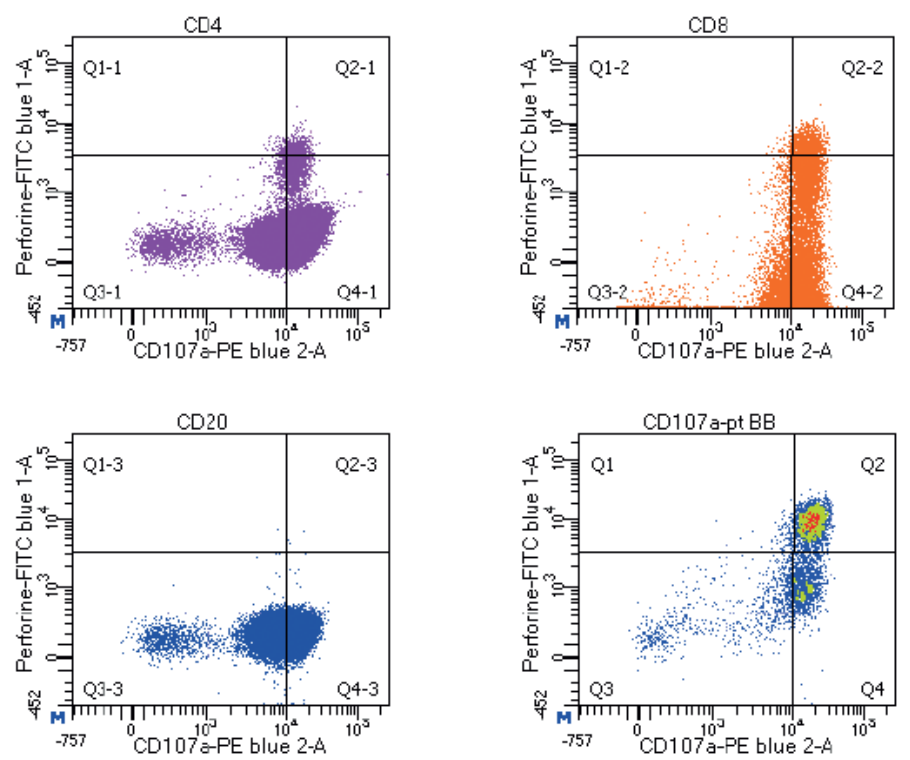
SUPPLEMENTAL FIGURE 3 | LAMP-1 EXPRESSION IN SIALIDOSIS TYPE I COMPARED TO CLN3-ASSOCIATED RETINAL DEGENERATION

\section{A | CLN3-ASSOCIATED RETINAL DEGENERATION}
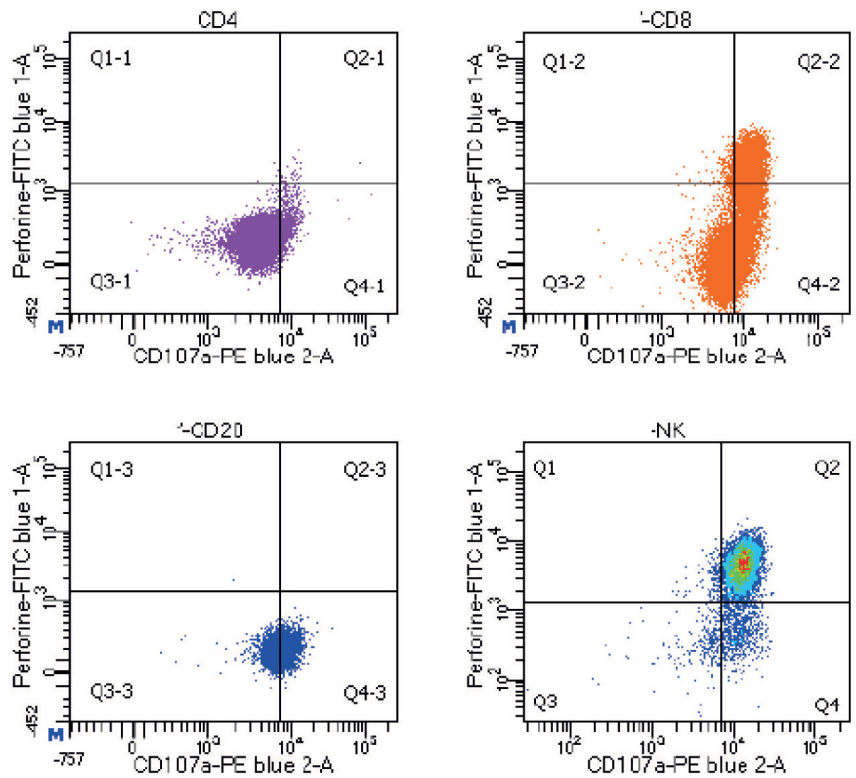

\section{B | SIALIDOSIS TYPE I}

BD FACSDiva 8.0.1
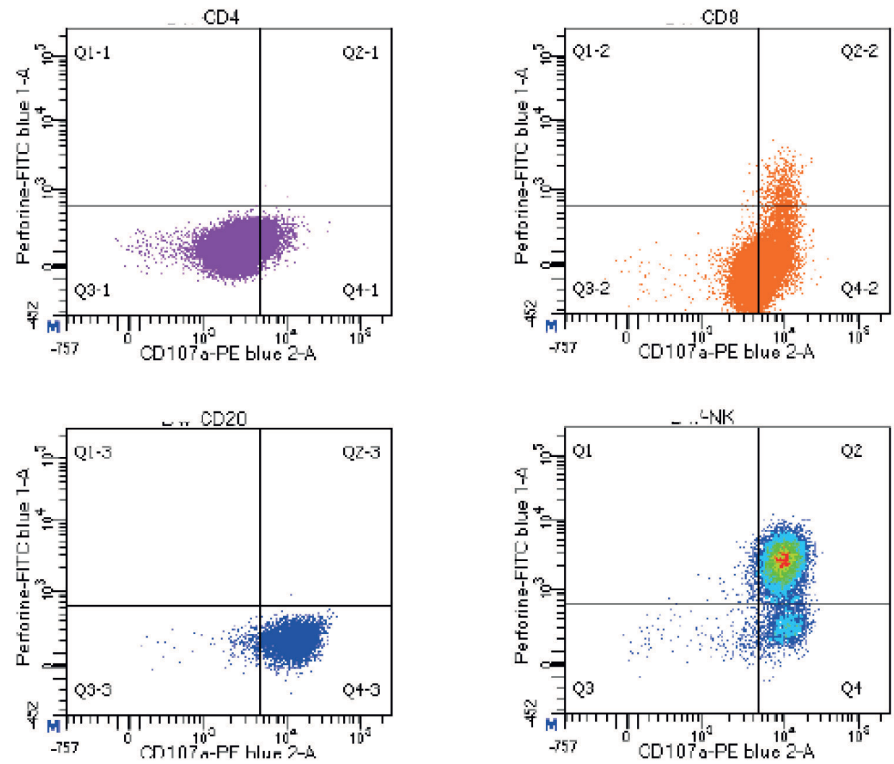



\section{CHAPTER STORAGE MATERIAL ACCUMULATION IN THE NEURONAL CEROID LIPOFUSCINOSES REVISITED}

Willemijn F.E. Kuper*, Holger Rehmann*, Peter M. van Hasselt

*These authors contributed equally to this work In progress

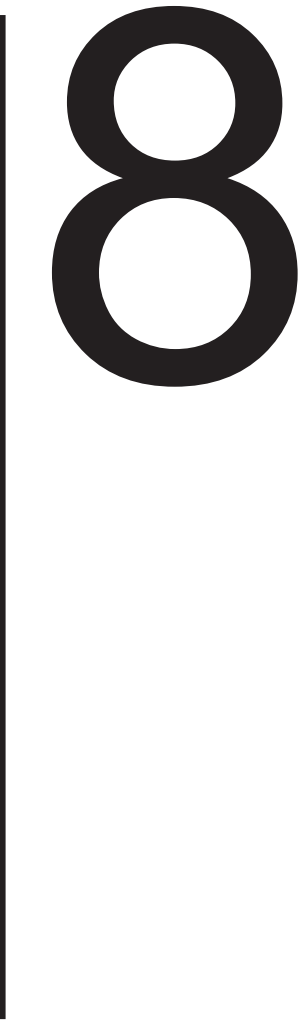




\section{ABSTRACT}

The neuronal ceroid lipofuscinoses (NCLs) are neurodegenerative disorders unified by the accumulation of auto fluorescent storage material. At this moment, the NCLs are considered as one group. However, they comprise more than 10 genetically distinct disorders associated with distinct storage material compositions. The pathophysiology of the NCLs is poorly understood. Current attempts to explain the pathophysiology consider the accumulation of the storage material as a byproduct of a general lysosomal imbalance. The storage material in the different NCLs consists however almost exclusively of either sphingolipid activator proteins (saposins) A and D, or subunit $\mathrm{C}$ of the mitochondrial ATP synthase. Here, we hypothesize that the CLN genes function in different steps of either saposins $A$ and $D$ or subunit c lysosomal degradation pathways, and present a model that explains the distinct compositions of the storage material. Following our hypothesis, we propose that the NCLs should be considered as two groups: the saposin-storing NCLs and the subunit c-storing NCLs. This mechanistic classification will hopefully help to understand pathophysiology and to ultimately treat these devastating disorders.

Key Words: neuronal ceroid lipofuscinosis (NCL); storage material accumulation; subunit c; sphingolipid activator proteins (saposins) A and D; pathophysiology 


\section{STORAGE MATERIAL ACCUMULATION IN THE NCLS FROM A HISTORICAL PERSPECTIVE}

The neuronal ceroid lipofuscinoses (NCLs) comprise a genetically heterogeneous group of disorders characterized by the premature and profound accumulation of auto fluorescent storage material and neuronal cell loss. ${ }^{1-3}$ Initially, the ceroid-lipofuscin like storage material accumulation was extensively studied in the hope that this shared feature would elucidate a shared pathophysiology. ${ }^{4-7}$ However, ever since some studies have suggested that the storage material is rather a non-specific byproduct of disease ${ }^{8}$, several other hypotheses have been explored ${ }^{9,10,11}$ to explain $\mathrm{NCL}$ pathophysiology. Currently however, NCL pathophysiology remains poorly understood.

There are several reasons to question the 'non-specific byproduct' hypothesis. First, as a 'non-specific byproduct', one would expect a heterogeneous mixture of accumulated storage material due to a general defect in the lysosomal degrading capacity. Rather, however, in the NCLs, the storage material consists almost exclusively of one specific component: either sphingolipid activator proteins (saposins) A and D (CLN1, CLN4 and CLN10), displayed as granular osmiophilic deposits (GRODs) under electron microscopy (EM), or subunit c of the mitochondrial ATPase (remaining NCLs) displayed as fingerprint profiles (FPPs) or curvilinear bodies (CVPs) under EM.,12-15 Second, in classical lysosomal storage diseases, the loss of an enzymatic activity results in the accumulation of the substrate normally converted by the enzyme. For example, in Tay-SachsDisease, $\beta$-N-Acetylhexosaminidase is deficient preventing the degradation of GM2-Ganglioside, which then accumulates. ${ }^{16}$ However, the protein degradation machinery in the lysosomes is non-specific and ultimately produces single amino acids, which are either reused for protein synthesis or are subjected to further catabolism. Proteins are cleaved stepwise to smaller and smaller peptides by several lysosomal proteases. Though each protease has a certain sequence specificity, this specificity is broad, and for example CLN10/CTSD cleaves peptide bonds between two hydrophobic residues. ${ }^{17}$ Thus, the loss of one protease may slow down protein degradation, which may result in accumulation of aggregated protein or protein fragments, but it should not result in the accumulation of one particular protein. By now more than 10 different genes are known to cause NCL. ${ }^{1}$ It may be considered unlikely that in all these different genetic subtypes this specific storage material accumulation is just a byproduct. Rather, the fact that either saposin A and D or subunit $c$ are 
accumulating strongly suggests that each of the NCLs can be either grouped in saposin or subunit c-storing.

\section{THE SAPOSIN STORING NCLS}

Peripheral membrane proteins are often modified post-translationally by attached lipids. The tail of the lipid is then inserted into membranes as an anchor to facilitate the membrane localization of the protein. Palmitoyl groups attached via an ester bond to the sulphur of a cysteine residue are one type of such anchors. ${ }^{18}$ CLN1/PPT1 was originally isolated from bovine brain due to its ability to de-palmitoylate the small G-protein $\mathrm{H}$-Ras, hence the name Palmitoyl-Protein Thioesterase 1 (PPT1). ${ }^{19}$ Lysosomal localisation of CLN1/ PPT1 was proven and it was assumed that PPT1 cleaves of palmitoyl groups from proteins or peptides during protein degradation. ${ }^{20}$ Indeed, it was shown by radioactive labelling techniques that small peptides with lipid modifications accumulate in lymphoblasts derived from CLN1 disease patients. Isolated storage material from 4 patients was shown to contain proteins and lipids and when resolved by SDS-PAGE one single dominant protein band of about $14 \mathrm{kDa}$ was observed. $\mathrm{N}$-terminal protein sequencing performed on this band resulted in the identification of saposin $A$ and saposin D. ${ }^{12}$

Saposin A, B, C, and D are generated by proteolytic cleavage from a single precursor protein and are thus formed in equal amounts. Saposins were originally identified as factors that stimulate the activity of several lipid hydrolysing enzymes. ${ }^{21}$ Saposins are thought to increase the exposure and accessibility of lipids to the hydrolysing enzymes. This concept is supported by the crystal structure of saposin $A$ in complex with the lipid analogue lauryldimethylamine- $\mathrm{N}$-oxide (LDAO). In this structure, two saposin $\mathrm{A}$ molecules are attached to a disc shaped "micro-micelle" of 40 LDAO molecules in a belt-like fashion. ${ }^{22}$ Consequently, the storage material in CLN1 patients should contain saposin A and saposin D bound lipid micelles. The palmitoylated peptides, which accumulate due to CLN1/PPT1 deficiency, are then anchored to these micelles. Thereby the palmitoylated peptides prevent the lipids in the saposin complex from degradation. Likewise, only those saposins accumulate, which are capable of forming these complexes. This type of storage material would then appear as GRODs at the level of EM. 
Since the storage material in the rare CLN10 and CLN4 subtypes has the same ultrastructural appearance as in CLN1, this may suggest a related defect. On the other hand CLN4 is the only dominantly inherited form of NCL, which may suggest a specific and distinct pathophysiological cause. ${ }^{23} \mathrm{CLN}_{4} / \mathrm{DNAJC5}^{+/-}$ drosophila are apparently normal whereas a semi-lethal phenotype and degradation of the synaptic termini of photoreceptors is observed in -/- flies. ${ }^{24}$ Likewise, +/- mice are normal but -/- mice suffer from degeneration of neuronal termini and die at 3 months of age. ${ }^{25}$ Both knock out models were generated prior to the identification of the CLN4 locus and an effect in +/- animals might have be missed due to lack of long term observation, but the available data do not support a dominant effect caused by haploinsufficiency. Furthermore, no GROD-like inclusions were noted in the ultrastructural analysis of neurons from the -/- animals. ${ }^{24,25} \mathrm{CLN} 4 / D N A J C 5$ does have a rather unique feature that offers a putative explanation for dominant inheritance. CLN4/DNAJC 5 contains a string of 13 cysteine residues of which 11 or 12 are palmitoylated. ${ }^{26}$ All three known disease causing mutations in CLN4 (UCL NCL mutation database) are localized within this string and the mutated protein is prone to aggregation. Newly synthesized CLN4/DNAJC5 may be delivered directly to the lysosome for degradation. Unusual high amounts of heavily palmitoylated protein then need to be degraded resulting in an overload of the CLN1/PPT1 dependent degradation process. An objection to this hypothesis is that in the course of normal protein turnover, also normal CLN4/DNAJC5 needs to be degraded and that thus the total amount of protein to be degraded remains the same. However, misfolded mutated CLN4/DNAJC5 may have a different entry point to lysosomal degradation. Furthermore, CLN4/DNAJC5 belongs to the heat shock family of chaperones, whose expression is upregulated in response to cellular stress, which may be caused by aggregated unfolded mutated CLN4/DNAJC5. This would result in a self-amplifying process. Finally, as the mutations reside in the cysteine string, mutated CLN4/DNAJC5 may be a sub-optimal substrate for CLN1/PPT1. Indeed, it has been shown that CLN1/PPT1 de-palmitoylates CLN4/DNAJC5 in vitro. ${ }^{27}$ 
FIGURE 1| PROPOSED PATHOPHYSIOLOGY OF THE SAPOSIN STORING NCLs

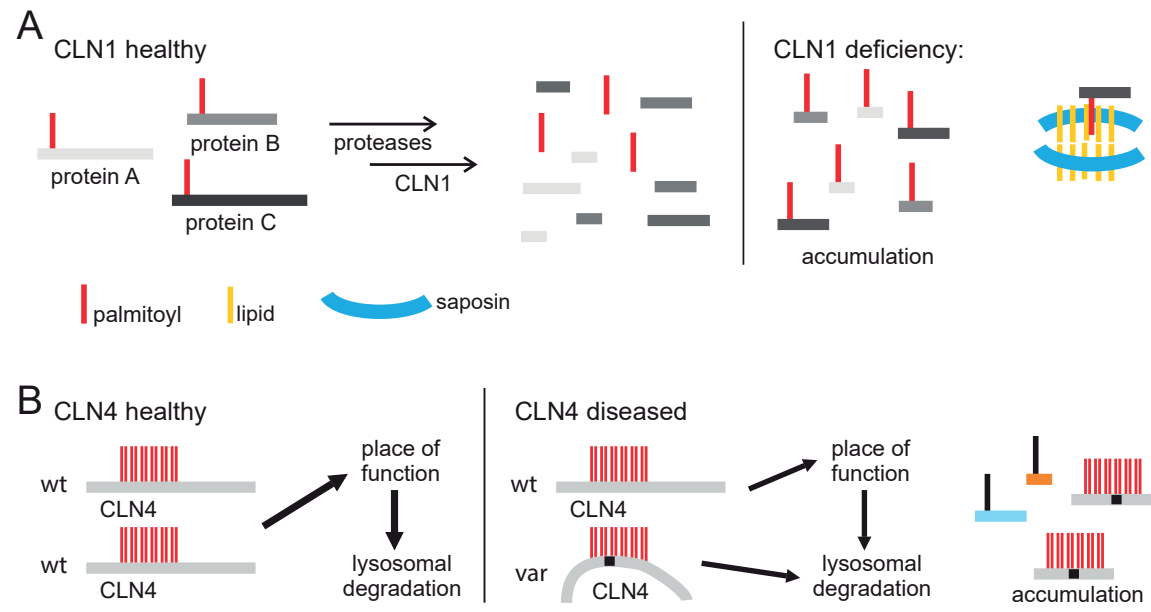

(A) Under healthy conditions CLN1/PPT1 de-palmitoylates proteins and peptides, which are in parallel degraded by proteases. CLN1/PPT1 deficiency results in the accumulation of short palmitoylated peptide fragments. These fragments can still be anchored to saposin solubilized lipid discs preventing the further processing of the saposin-lipid complex. (B) Under healthy conditions CLN4 is produced from both alleles resulting in functional CLN4 protein with a heavily palmitoylated cysteine rich string. The pathogenic variants carry a mutation in this cysteine rich string. The affected allele produces an instable, aggregation-prone protein that is subjected to degradation by lysosome, where the mutated palmitoylated cysteine rich string is inefficiently de-palmitoylated resulting in a similar accumulation of palmitoylated peptide bound to saposin-lipid complexes as shown in $(A)$.

\section{THE SUBUNIT C STORING NCLS}

Subunit c is one of about 15 different proteins that constitute the mitochondrial ATP synthase complex. The complex spans the inner mitochondrial membrane and appears in EM images as mushroom-like structures tightly decorating the membrane. The synthesis of ATP from ADP and phosphate requires conformational changes within the ATP synthase caused by a continuing rotation of parts of the complex. The rotation and thereby the formation of ATP is energetically driven by protons, which enter the mitochondrial matrix from the intermembrane space by passage through the synthase complex. As the proton gradient is created as a consequence of the catabolic activity 
of the cell, ATP synthase ensures that catabolically generated energy can be used for anabolism. As ATP synthesis is the central function of mitochondria, the ATP synthase complex is highly abundant. ${ }^{28}$

Each ATP synthase complex contains 8 molecules of processed subunit c. Processed subunit $\mathrm{c}$ is a small protein of 75 amino acid residues with a calculated molecular mass of $7.6 \mathrm{kDa}$ that forms a helix-loop-helix structure (Figure 2). The helices span the membrane and the $8 \mathrm{c}$-subunits form a ring that is filled with lipids. Thus, both the outwards as well as the inwards facing helices are in contact with membrane. ${ }^{29}$

The continuous renewing of mitochondria requires the degradation of old or damaged mitochondria. To this end, mitochondria are encapsulated to form autophagosomes, which are subsequently fused to lysosomes. This allows the degradation of complete mitochondria. ${ }^{30}$ Subunit c likely forms a challenge to the lysosomal degradation machinery. The ring of $8 \mathrm{c}$-subunits is tightly embedded in the mitochondrial membrane and thus not easily assessable to proteases. For the degradation of "normal" transmembrane proteins, proteases likely start cleaving in those parts of the proteins that extend from the membrane, so that the connectivity between single transmembrane helices get lost. For the degradation of ATP synthase, cleaving in those parts extending from the membrane would likely result in the degradation of most subunits of the complex, but leaves the compact ring of the 8 $\mathrm{c}$-subunits intact. The remaining ring of the $\mathrm{c}$-subunit then still forms a stable complex without protease accessible parts extending from the membrane. It is thus likely that this ring requires some kind of active extraction from the membrane. Furthermore, subunit $c$ is extremely hydrophobic. While organic solvents such as phenol/chloroform are traditionally used to precipitate protein from cell lysates for example in course of DNA isolation, subunit c is soluble in chloroform/methanol. ${ }^{30}$ As this is a feature typical for lipids but very uncommon for proteins, some early researchers invented the term "proteolipids" for such compounds. ${ }^{31}$ It is thus likely that a chaperone system is required to solubilize c-subunit after its extraction from the mitochondrial membrane. To summarize, a combination of high secondary structure stability, uncommon sequence features associated with the extreme hydrophobicity, and the short protein length may make subunit $\mathrm{c}$ a bad substrate for most proteases. 


\section{HYPOTHESIS}

We therefore propose a multi-step process of subunit c degradation and suggest that different steps herein are mediated by different CLN genes. For example, CLN3 might be involved in the extraction of the ring from the membrane. Under healthy conditions, general transmembrane proteins are degraded, the subunit c ring is extracted and in parallel lipids are removed from the membrane to disassemble the former mitochondrial structure. If subunit c extraction is interrupted, subunit c will accumulate in shrinking membrane space until membrane structures tightly loaded with subunit c remain from which no further lipids can be removed. This hypothetical membrane structure might explain the fingerprint profile of the storage material observed in CLN3 disease. ${ }^{15}$ Other CLNs might solubilize subunit c after membrane extraction. CLN2 (TPP1) might initiate the proteolytic degradation of subunit c. The removal of $\mathrm{N}$-terminal amino acids might destabilize the helical fold and thereby make subunit $\mathrm{c}$ assessable to other proteases. If this is not possible due for example CLN2 deficiency, CLN3 may aggregate and such aggregates may appear as curvilinear structures in EM.

This model does not require that CLN proteins are exclusively involved in subunit c degradation. For example, CLN2/TPP1 has a broad substrate specificity and may thus act on many proteins and protein fragments. ${ }^{32}$ Normally, other proteases can compensate for the loss of CLN2/TPP1 activity as no strict order of cleavage reactions is required to degrade these proteins. However, due to the specific characteristics of subunit c, the CLN2/TPP1 catalyzed step is a bottleneck that cannot be circumvented by other enzymes. The rather high subunit c content of mitochondria adds a further challenge to the bottleneck of the limited degradation process. Furthermore, this model can explain why in certain NCL mixtures of different storage material is observed. If a gene involved in a later step of the degradation process is affected, a "tailback" situation may occur where also previous steps in the degradation process derail, causing a mixture of e.g. CVPs and FPPs on EM.

A consequence of this view is to consider the storage material as being pathogenic causative for the disease. Increasing accumulation of storage material likely increasingly interferes with normal lysosomal function. This would also impinge on the formation and maturation of lysosomes and thereby affect the equilibria between membrane compartments. Upon massive accumulation the structure of membrane compartments, as for example of 
the Golgi complex, may in part get lost. This massive disturbances would then ultimately result in cell death. In neurons, disturbances of vesicle transport from the soma to the axon and dendrites may also result in axon and dendrite degeneration.

The predominant neurological manifestations of the NCLs as a direct result of storage material accumulation can be explained by two lines of arguments. First, the regenerative power of the central nervous system is extremely limited. The loss of a rather limited number of neurons is already sufficient to disturb neuronal networks. ${ }^{33}$ Second, storage material accumulation in dividing and regenerating tissues may be slower. In these tissues, cell division would reduce storage burden per cell. Furthermore, in particular during mitosis lysosomal endocytosis may result in the expulsion of storage material. In general, "waste disposal" by lysosomal endocytosis may be more common in non-neuronal tissues, where the expelled "waste" can be processed more easily than behind the blood-brain-barrier. Interestingly, increased levels of subunit c were detected in the urine of an NCL patient, tough the source of it could well be dying kidney cells instead of an "expulsion" process. ${ }^{34}$ 


\section{FIGURE 2 | PROPOSED PATHOPHYSIOLOGY OF THE SUBUNIT C ACCUMULATING NCLS}
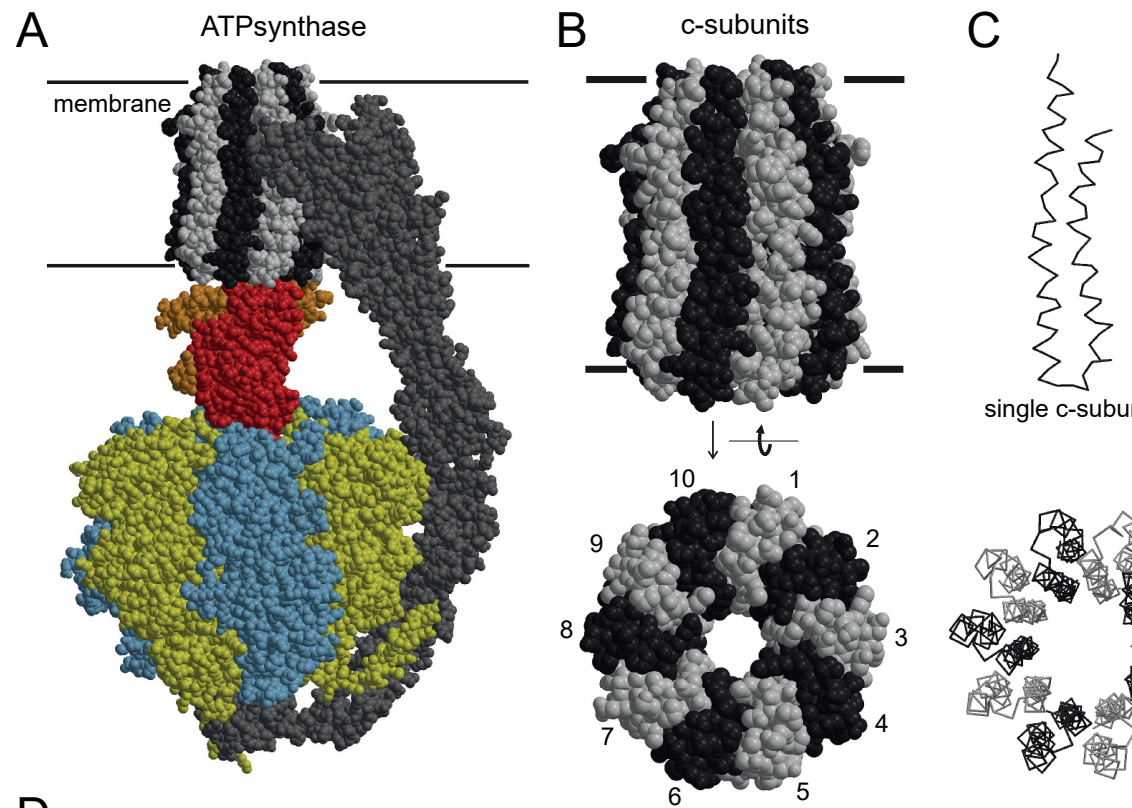

$\mathrm{D}$

lysosomal degradation of mitochondria

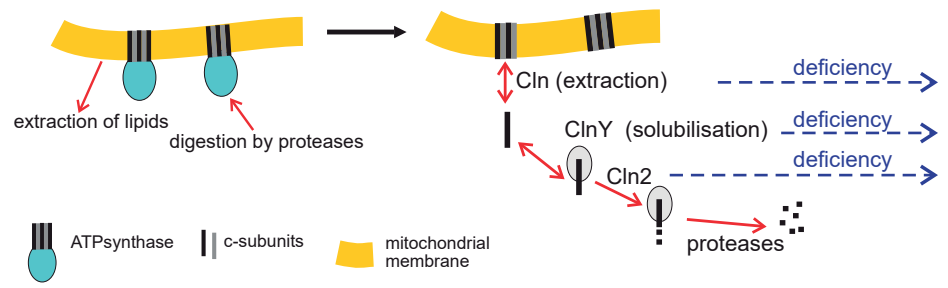

storage material:

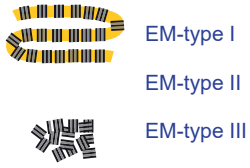

(A) Space filling model of ATP synthase from yeast (pdb entry 6cp6). The localization of the membrane is indicated. Colors code for different proteins or sub-complexes. (B) Magnification of c-subunit ring as in (A) (upper panel) and after $90^{\circ}$ tilt (lower panel). Individual c-subunits are colored alternatingly in light and dark grey. Human ATP synthase displays a similar architecture but the c-subunit ring consists of 8 instead of 10 subunits. (C) Single c-subunit shown as backbone trace visualizing the helix-loop-helix structure (upper panel) and the whole ring (lower panel). (D) Proposed steps for the degradation of ATP synthase in the lysosome. Components of ATP synthase extending from the membrane are degraded by proteases, whereas the hardly accessible c-subunit requires an active extraction from the membrane. Malfunctioning of this process due to CLN deficiencies result in accumulation of c-subunit in different stages with specific EM appearances. 


\section{CONCLUSIONS}

As shown above, it seems possible to explain the accumulation of either subunit c or saposins A and D by "specific" defects in lysosomal functions. The proposed defects match the function of the genes as far as the functions are known as we have discussed for example for the protease activity of CLN2/TPP1. In cases were the function of the gene is unknown, proposed functions that could explain the nature of the storage material are in agreement with known properties of the gene. For example, CLN3 is very likely a transmembrane protein that resides in the lysosome. ${ }^{35}$ As such it could fulfil a kind of chaperone activity to extract subunit c from the membrane. Loss of this proposed activity could explain the typical fingerprint profile of the storage material observed in CLN3 disease. It is then the massive accumulation of the storage material that causes cell dysfunction and death that the neuronal tissues are particularly vulnerable to.

In consequence, we propose that the NCLs should be classified as either saposin or subunit c storing as the NCLs within these groups seem to be related by the underlying pathophysiology. This concept offers a different view on the NCLs that may help to better understand these disorders and ultimately develop treatment strategies. 


\section{REFERENCES}

${ }^{1}$ Mole SE, Cotman SL. Genetics of the neuronal ceroid lipofuscinoses (Batten disease). Biochim Biophys Acta. 2015 Oct;1852(10 Pt B):2237-41.

${ }^{2}$ Kousi M, Lehesjoki AE, Mole SE. Update of the mutation spectrum and clinical correlations of over 360 mutations in eight genes that underlie the neuronal ceroid lipofuscinoses. Hum Mutat. 2012 Jan;33(1):42-63.

${ }^{3}$ Haltia M. The neuronal ceroid-lipofuscinoses. J Neuropathol Exp Neurol. 2003 Jan;62(1):1-13.

${ }^{4}$ Kominami E, Ezaki J, Muno D, Ishido K, Ueno T, Wolfe LS. Specific storage of subunit c of mitochondrial ATP synthase in lysosomes of neuronal ceroid lipofuscinosis (Batten's disease). J Biochem. 1992 Feb;111(2):278-82.

${ }^{5}$ Ezaki J, Wolfe LS, Higuti T, Ishidoh K, Kominami E. Specific delay of degradation of mitochondrial ATP synthase subunit $c$ in late infantile neuronal ceroid lipofuscinosis (Batten disease). J Neurochem. 1995 Feb;64(2):733-41.

${ }^{6}$ Palmer DN, Fearnley IM, Walker JE, Hall NA, Lake BD, Wolfe LS, Haltia M, Martinus RD, Jolly RD (1992) Mitochondrial ATP synthase subunit c storage in the ceroid-lipofuscinoses (Batten disease). Am J Med Genet 42 : 561-567

${ }^{7}$ Palmer DN, Bayliss SL, Westlake VJ. Batten disease and the ATP synthase subunit c turnover pathway: raising antibodies to subunit c. Am J Med Genet. 199557 : 260-265

${ }^{8}$ Palmer DN. The relevance of the storage of subunit c of ATP synthase in different forms and models of Batten disease (NCLs). Biochim Biophys Acta. 2015 Oct;1852(10 Pt B):2287-91.

${ }^{9}$ Grünewald B, Lange MD, Werner $C$ et al. Defective synaptic transmission causes disease signs in a mouse model of juvenile neuronal ceroid lipofuscinosis. Elife. 2017 Nov 14;6. pii: e28685

${ }^{10}$ Segal-Salto M, Hansson K, Sapir T. Proteomics insights into infantile neuronal ceroid lipofuscinosis (CLN1) point to the involvement of cilia pathology in the disease. Hum Mol Genet. 2017 May 1;26(9):1678.

${ }^{11}$ Cárcel-Trullols J, Kovács AD, Pearce DA. Cell biology of the NCL proteins: What they do and don't do. Biochim Biophys Acta. 2015 Oct;1852(10 Pt B):2242-55.

${ }^{12}$ Tyynela J, Palmer DN, Baumann M, Haltia M. Storage of saposins A and D in infantile neuronal ceroid-lipofuscinosis. FEBS Lett 330, 8-12 (1993).

${ }^{13}$ Nijssen PC, Ceuterick C, van Diggelen OP et al. Autosomal dominant adult neuronal ceroid lipofuscinosis: a novel form of NCL with granular osmiophilic deposits without palmitoyl protein thioesterase 1 deficiency. Brain Pathol. 2003 Oct;13(4):574-81.

${ }^{14}$ Marques ARA, Di Spiezio A, Thießen $\mathrm{N}$ et al. Enzyme replacement therapy with recombinant pro-CTSD (cathepsin D) corrects defective proteolysis and autophagy in neuronal ceroid lipofuscinosis. Autophagy. $2019 \mathrm{Jul}$ 16:1-15.

${ }^{15}$ Anderson GW, Goebel HH, Simonati A. Human pathology in NCL. Biochim Biophys Acta. 2013 Nov;1832(11):1807-26.

${ }^{16}$ Mahuran DJ. Biochemical consequences of mutations causing the GM2 gangliosidoses. Biochim Biophys Acta. 1999 Oct 8;1455(2-3):105-38.

${ }^{17}$ Vidoni C, Follo C, Savino M, Melone MA, Isidoro C. The Role of Cathepsin D in the Pathogenesis of Human Neurodegenerative Disorders. Med Res Rev. 2016 Sep;36(5):845-70.

${ }^{18}$ Linder ME, Deschenes RJ. Palmitoylation: policing protein stability and traffic. Nat Rev Mol Cell Biol. 2007 Jan;8(1):74-84.

${ }^{19}$ Camp LA, Hofmann SL. Purification and properties of a palmitoyl-protein thioesterase that cleaves palmitate from H-Ras. J Biol Chem. 1993 Oct 25;268(30):22566-74. 
${ }^{20}$ Verkruyse LA, Hofmann SL. Lysosomal targeting of palmitoyl-protein thioesterase. J Biol Chem. 1996 Jun 28;271(26):15831-6.

${ }^{21}$ Kishimoto Y, Hiraiwa M, O’Brien JS. Saposins: structure, function, distribution, and molecular genetics. J Lipid Res. 1992 Sep;33(9):1255-67.

22 Popovic K, Holyoake J, Pomes R, Prive GG. Structure of saposin A lipoprotein discs. Proc Natl Acad Sci U S A 109, 2908-2912, doi:10.1073/pnas.1115743109 (2012).

${ }^{23}$ Nosková L, Stránecký V, Hartmannová H et al. Mutations in DNAJC5, encoding cysteine-string protein alpha, cause autosomal-dominant adult-onset neuronal ceroid lipofuscinosis. Am J Hum Genet. 2011 Aug 12;89(2):241-52.

${ }^{24}$ Zinsmaier KE, Eberle KK, Buchner E, Walter N, Benzer S. Paralysis and early death in cysteine string protein mutants of Drosophila. Science 263, 977-980, doi:10.1126/science.8310297 (1994).

${ }^{25}$ Fernandez-Chacon R, Wölfel M, Nishimune $\mathrm{H}$ et al. The synaptic vesicle protein CSP alpha prevents presynaptic degeneration. Neuron 42, 237-251 (2004).

${ }^{26}$ Gundersen CB, Mastrogiacomo A, Faull K, Umbach JA. Extensive lipidation of a Torpedo cysteine string protein. J Biol Chem 269, 19197-19199 (1994).

${ }^{27}$ Henderson MX, Wirak GS, Zhang YQ et al. Neuronal ceroid lipofuscinosis with DNAJC5/ CSPalpha mutation has PPT1 pathology and exhibit aberrant protein palmitoylation. Acta Neuropathol 131, 621-637, doi:10.1007/s00401-015-1512-2 (2016).

${ }^{28}$ Stock D, Leslie AG, Walker JE. Molecular architecture of the rotary motor in ATP synthase. Science. 1999 Nov 26;286(5445):1700-5.

${ }^{29}$ Girvin ME, Rastogi VK, Abildgaard F, Markley JL, Fillingame RH. Solution structure of the transmembrane $\mathrm{H}+$-transporting subunit c of the F1F0 ATP synthase. Biochemistry. 1998 Jun 23;37(25):8817-24.

${ }^{30}$ Youle RJ, Narendra DP. Mechanisms of mitophagy. Nat Rev Mol Cell Biol. 2011 Jan;12(1):9-14.

${ }^{31}$ Folch J, Lees M. Proteolipides, a new type of tissue lipoproteins; their isolation from brain. J Biol Chem. 1951 Aug;191(2):807-17.

32 Tian Y, Sohar I, Taylor JW, Lobel P. Determination of the substrate specificity of tripeptidylpeptidase I using combinatorial peptide libraries and development of improved fluorogenic substrates. J Biol Chem. 2006 Mar 10;281(10):6559-72.

${ }^{33}$ Ahrens-Nicklas RC, Tecedor L, Hall AF et al. Neuronal network dysfunction precedes storage and neurodegeneration in a lysosomal storage disorder. JCI Insight. 2019 Nov 1;4(21).

${ }^{34}$ Wisniewski, K. E., Golabek, A. A. \& Kida, E. Increased urine concentration of subunit c of mitochondrial ATP synthase in neuronal ceroid lipofuscinoses patients. J Inherit Metab Dis 17, 205-210, doi:10.1007/bf00711619 (1994).

${ }^{35}$ Mirza M, Vainshtein A, DiRonza A et al. The CLN3 gene and protein: What we know. Mol Genet Genomic Med. 2019 Dec;7(12):e859. 



\section{CHAPTER \\ GENERAL DISCUSSION}

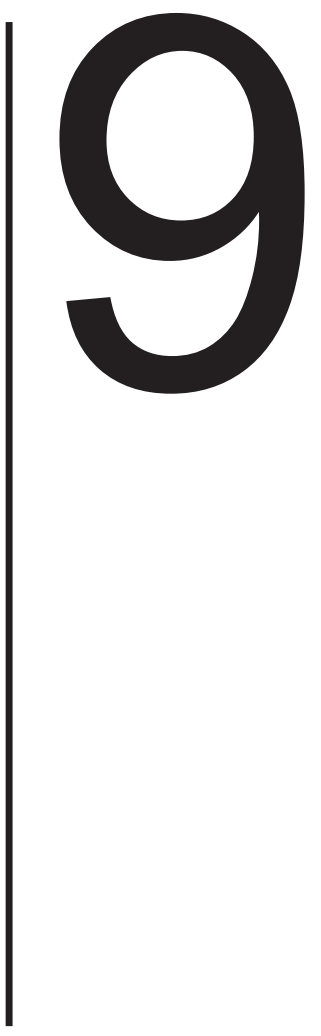




\section{BEYOND THE BLIND SPOTS}

In the general introduction, we stated that the lack of understanding of Batten disease could be compared to the existence of blind spots. In this chapter, the main findings of this thesis are discussed in the context of these blind spots. Have we been able to look beyond them? But also, which blind spots might still exist or might have even developed?

\section{BEYOND OUR BLIND SPOTS}

You can only see it when you get it

As Johan Cruyff put it, "je gaat het pas zien als je het door hebt" (you can only see it when you get it). For a long time, and sometimes even still, research articles on Batten disease state somewhere in the first few sentences that vision loss precedes neurodegeneration, generally by a few years. ${ }^{1,2}$ These first few sentences in an introduction are meant to state what we already know, followed by a statement of what we do not yet know (and thus what that study will explore). Due to their position early in the introduction, the initial statements of what we already know are just read over and generally not questioned. To be able to look beyond such (potential) blind spots, generally starts with the uncomfortable hunch that what you think is actually happening, might be different. The research performed in Part $\mathbf{1}$ of this thesis started with the hunch that what we read on the onset of vision loss being followed by cognitive decline did not correspond with our clinical experience. In our practice, patients with CLN3 disease would often have learning difficulties at disease onset already. For each individual patient, these learning problems were attributed to their dramatic loss of vision. By performing a meta-analysis of published case reports, supplemented with the medical files from our own patients, we were able to confirm that cognitive impairment has its onset early in (classical) CLN3 disease, which cannot be explained by the co-occurring vision loss (Chapter 2). As we also demonstrated early onset of motor function impairment (Chapter $\mathbf{3}$ ), we concluded that neurodegeneration has its onset early in (classical) CLN3 disease. Currently, in our practice, early learning difficulties in a school-aged child with retinal dystrophy, are perceived as an important cue for CLN3 disease. This improves early recognition, as well as tailored counseling following diagnosis, since these patients require from early on a different approach than practical adjustments for their visual impairment 
alone. Moreover, the IQ scores and 6MWT scores might provide relevant clinical markers to monitor disease progression. Future research may possibly provide more specific, clinical markers for disease progression. Along these lines, we have noted that numeracy skills seem to deteriorate before language and literacy skills (unpublished data). Perhaps this, and other tell-tale signs will provide an even more detailed insight in the course of the neurological decline.

Despite its early onset, the initial signs of neurologic impairment are quite subtle, particularly when compared to the overwhelming vision loss. Hence, patients generally do not meet the pediatrician or geneticist first, but rather the ophthalmologist. We therefore realized that, to actually improve early recognition, we needed to help the ophthalmologist to distinguish CLN3 disease from a retina-only disease which both appear similar at first sight. Moreover, the rarity of CLN3 disease particularly precludes the general ophthalmologist to recognize this disease on clinical experience, a process called pattern recognition. In Chapter 4 we hypothesized that we could mimic pattern recognition using a deep phenotyping approach. Indeed, following an in-depth analysis of ophthalmological test results, we were able to identify several differentiating characteristics of CLN3 disease around first presentation at the ophthalmologist. These differentiating characteristics were - once we got a clear picture - easily recognizable in any individual patient. You can only see it when you get it.

You have to know where to look

Following Part 1 of this thesis, it has become clear that recognizing a rare disorder, such as CLN3 disease, can be as easy as recognizing a more common disorder, if you only know where to look. For a rare disorder, however, you need to take a different approach to get the complete picture in view - an approach one could refer to as 'epidemiology of the rare'. In this approach, centralization is essential since only pooling of patients allows you to see patterns that you would not have identified in a single patient beforehand (yet, based on the analysis of the group you can identify these patterns in a single patient afterwards). Still, when centralizing care for, and research of rare disorders, patient numbers will remain inherently small. A major component of the epidemiology of the rare is to create artificially large cohorts that consist of (all) published cases, if possible supplemented with unpublished medical records, such as performed in Chapter 2, and as previously performed by 
our group and others. ${ }^{3,4}$ Retrospective data research does have its inherent limitations, such as data collection performed in a non-standardized manner, with missing data. Still, meta-analyses of case reports can help to gain new insights in rare disorders that one would not, or only significantly later, have gained with prospective research. We therefore believe that it is of utmost importance to keep medical records as detailed, and as up to date as possible, as to continue to publish detailed case reports and case series to help build these artificial cohorts. Although case reports and case series may not represent the highest order of evidence, they generally do represent the first line of evidence - often describing an unexpected aspect of disease for the first time. ${ }^{5}$ To be able to notice the unexpected may be the first step towards new insights - but only follows after having learned where to look.

\section{Seeing additional shades of grey}

Previously, the phenotype of CLN3 disease was regarded rather dichotomous: patients displayed either classical CLN3 disease - associated with childhood onset neurodegeneration - or protracted CLN3 disease - associated with adult onset neurodegeneration. ${ }^{6,7}$ We challenged this view in Chapters 5 and 6, describing patients that did not fit this dichotomous division. Following the results of Part $\mathbf{2}$ of this thesis, we rather propose that the phenotypic severity of CLN3 disease is distributed among a continuous spectrum - as visualized in Figure 1. The patients described in Chapter 5 underline the potential danger of blind spots in assessing disease severity, particularly in light of (near) future therapeutic efficacy studies. We want to underline that prudence is warranted when classifying patients with an atypical genotype as having classical CLN3 disease, even if supported by impaired cognitive and/or motor test scores early in the disease. Based on the disease course of two of the patients described in Chapter 5, we propose that protracted CLN3 disease, in some patients, may initially have an early onset of neurodegeneration similar to classical CLN3, with the delayed decline only becoming clear in the following years. If (falsely) classified as classical CLN3 disease, false positive effects of a therapy might be reported. This is a pitfall in small cohorts particular, and it might have happened in a previous study. ${ }^{8}$ In this study, that tested the efficacy of an anti-inflammatory treatment modality in a total of 8 CLN3 disease patients, the verbal IQ was found to increase in two of the younger patients. However, one of the patients had the E295K missense mutation on one allele, which has consistently been associated with protracted CLN3 disease, more likely 
explaining this apparent effect., ${ }^{6,7}$ The authors did report that long-term followup studies would be needed, but classifying patients as accurately as possible, from early on, might allow to determine therapeutic efficacy quicker and more reliably.

FIGURE 1 | A NEW VIEW ON THE PHENOTYPIC SPECTRUM OF CLN3 DISEASE

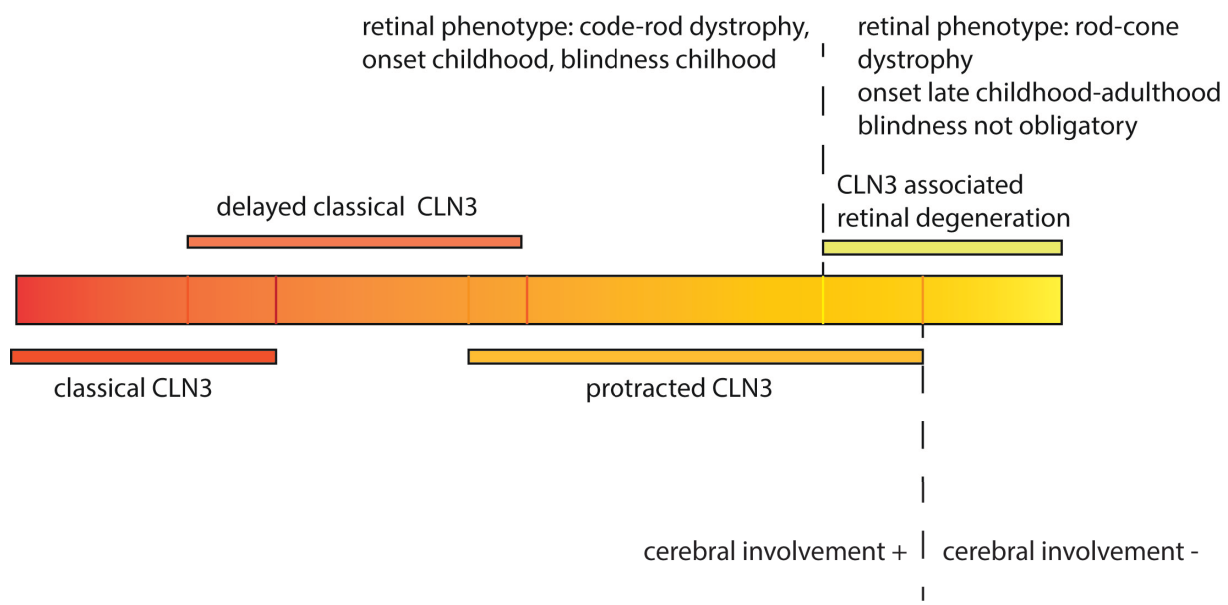

A graphical overview of the phenotypic spectrum of CLN3 disease, ranging from classical CLN3 disease to CLN3-associated (isolated) retinal degeneration

At the very mild end of the clinical spectrum, putatively a relatively high degree of residual CLN3 protein function leads to a retinal and neurological phenotype that is markedly different from classical - protracted CLN3 disease. In classical up to and including protracted CLN3 disease, patients suffer from an earlyonset cone-rod dystrophy which quickly progresses into blindness. Timing and rate of progression of the retinal phenotype vary little between patients. ${ }^{9-11}$ Neurologically, timing and rate of progression vary significantly between patients. The neurological phenotype may range from childhood onset of decline and death in early adulthood in classical CLN3 disease, to adult onset of decline and death upon middle age in protracted CLN3 disease. Even between patients displaying the same phenotype, several neurological nuances are seen, e.g. early onset but slow progression of neurologic decline in some cases of protracted CLN3 disease as described in Chapter 5. Previously, the tipping point between cone-rod dystrophy and rod-cone dystrophy was suggested to align neatly with the tipping point between the presence or absence of 
neurological deterioration, respectively. ${ }^{12,13}$ However, in Chapter 6, we argued that these tipping points do not exactly align, as some of the CLN3-associated (isolated) retinal degeneration patients do suffer from neurological decline, underscoring the continuous character of the CLN3 disease phenotypic spectrum (Figure 1). Although this thesis has explained some aspects of the phenotypic variability following bi-allelic mutations in CLN3, future research is needed to elucidate the remaining - possibly predominantly non-genetic - modifiers of disease. Until then, prudency is warranted when counseling patients, particularly those at the tipping point between presence or absence of neurological decline, which severely impacts life.

Since the CLN3-associated (isolated) retinal degeneration phenotype is so different from classical-protracted CLN3 disease, the contents of Part 1 of this thesis, on early recognition of Batten disease, are not directly applicable to these retina-only patients. However, we believe that the deep phenotyping approach that was used is suitable to similarly delineate this phenotype as well. Future efforts are needed to determine how (ultra)rare this phenotypic subgroup is exactly. From the perspective of CLN3 disease, we regard CLN3 associated retinal degeneration as a rare phenotype of an already rare disease. Yet, from the perspective of visual impairment in general, it remains to be elucidated which percentage of the visually impaired/blind adults (due to retinal dystrophy) actually have this alternative phenotype of CLN3. With respect to the complete CLN3 disease spectrum, this retina-only phenotype probably resembles what we can achieve with high-end residual protein function stimulating drugs until we can completely restore normal CLN3 protein function. Based on what we know from other LSDs, most likely only a slight increase in protein level will already have a major impact on the phenotype.

\title{
FUTURE PERSPECTIVES
}

\section{BEYOND BATTEN DISEASE}

\author{
REVISITING BATTEN DISEASE
}

With increasing insight in Batten disease, its complexity is also becoming increasingly clear. ${ }^{14}$ Currently, more than 10 different CLN proteins are identified that do not share clear similarities, other than a (presumed) 
lysosomal location of most of the proteins. This increasing complexity has even led some researchers to question why we still consider the NCLs as one group. ${ }^{15}$ These authors rather argued that the NCLs are fundamentally different disorders, only emerging on similar end points such as a general lysosomal misbalance. Indeed, already in 1969, when Zeman and Dyken proposed the term NCL, they stated that this classification was only "until further research permits a more meaningful classification". ${ }^{16}$ Currently, the individual NCLs are named according to their genetic defect, which is a logical and durable choice. ${ }^{17}$ We do however agree that the overarching classification deserves an update, which we would propose to base on the new insights in storage material accumulation as discussed in Part $\mathbf{3 .}$

In Chapter 7, we provide data that strongly suggest a direct link between the degree of storage material accumulation and the degree of CLN3 disease severity. This urged us to rethink the importance of the storage material in CLN3 disease and other NCLs. According to the current paradigm, storage material is a nonspecific consequence of lysosomal dysfunction. ${ }^{18}$ In Chapter $\mathbf{8}$, we argued that a heterogeneous mixture of storage materials would be expected if this were the case. This is however not the case. Rather, the NCLs almost exclusively accumulate saposins $A$ and $D$ or subunit $c$. This led us to hypothesize that the CLN genes function in different steps of either the saposins $A$ and $D$ or subunit $c$ lysosomal degradation pathway. This in turn implies that the position of each of the CLN proteins in these pathways, likely explains the different storage material compositions as visible under EM in case of a CLN deficiency. Following our hypothesis, we would propose - at least until further research permits a more meaningful classification - that the NCLs can best be divided in two groups: the saposin-storing NCLs and the subunit c-storing NCLs.

Our hypothesis that defective degradation of intracellular material is the cardinal feature in both the saposin-storing NCLs and the subunit c-storing NCLs leaves room for a pathophysiological link between the NCLs and autophagy, the intracellular degradation of damaged or redundant cellular components. ${ }^{19}$ In support, autophagy is particularly relevant to guard cellular homeostasis in non-dividing and metabolically active cells, such as neurons and retinal cells. Recently, Teinert et al provided a comprehensive overview of the congenital disorders of autophagy, an emerging group of IEMs. ${ }^{19}$ Progressive neurodegeneration is a key clinical feature in all of these disorders, 
and in some, storage material accumulates. This includes SPG11 deficiency. ${ }^{20}$ SPG11 functions in the final steps of autophagy and when SPG11 is deficient, enlarged lysosomes accumulate - similar as we demonstrated for CLN3 disease in Chapter 7. Characteristic features in SPG11 include motor decline, cognitive decline and retinal degeneration - clearly similar to the features seen in NCL. Interestingly, a bi-allelic micro-deletion in SPG11 was identified in a patient who was referred to our clinic with a suspicion of an NCL, based on the neurological and retinal deterioration in addition to electron dense accumulation of storage material, somewhat resembling the FPPs as seen in CLN3 disease. This case supports the hypothesis that defective autophagy is a key component in NCL. In this regard it is relevant to mention that enhancement of autophagy via transcription factor EB (TFEB) delayed disease progression in CLN3 disease mice. ${ }^{21}$ Currently, efforts are being undertaken to test this route of autophagy enhancement in human CLN3 disease patients (BBDF-101 via the Beyond Batten Disease foundation).

\section{FIGURE 2 | OVERVIEW OF THE (CURRENTLY PROPOSED) NCLS AND THE ASSOCIATED CLN GENES AND PROTEINS}

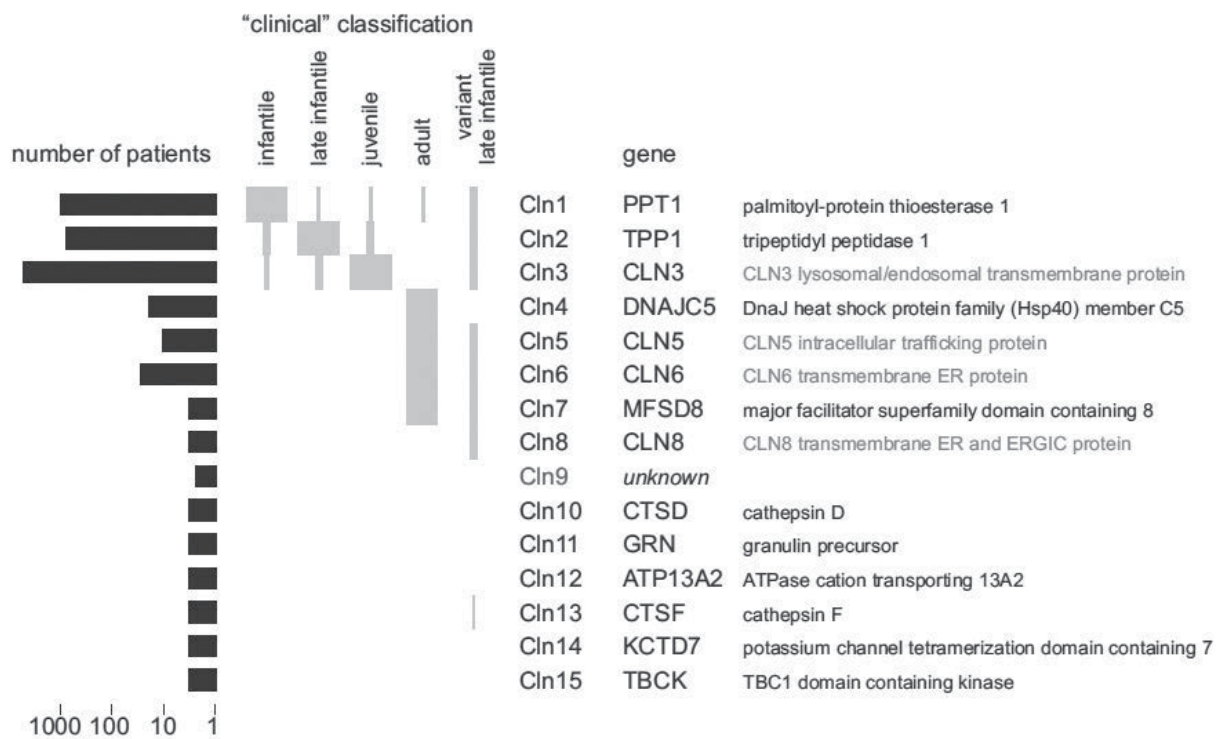

Graphical overview of the rough total of currently reported patients per NCL subtype, the main clinical phenotypes, the respective genes and the respective proteins. 


\section{BEYOND THE UNTREATABLE - TOWARDS TREATMENT OPTIONS}

The search for and hopefully identification of treatment options might come with some (potential) difficulties that we should not overlook. The early potential treatment strategies for CLN3 disease may delay disease progression but are unlikely to completely stop progression. Even gene therapy, as currently being developed to specifically target neurons, is not expected to cure the disease, as it is increasingly recognized that other cell types are actively involved in the disease process as well. ${ }^{22,23}$ This includes cell types both within the (central) nervous system and outside the (central) nervous system..$^{24}$ Particularly when the delaying treatment options will arrive, these peripheral manifestations, that currently do not seem clinically relevant, might actually become relevant manifestations of disease..$^{25}$

Although this thesis is aimed at gaining understanding of the underlying pathophysiology, one could question whether this is necessary to treat Batten disease. Recently, a hyper-individualized gene therapy approach - suitable for one girl (Mila) with one specific mutation in $C L N 7^{26}$ - was developed in a couple of months, without an extensive understanding of the pathophysiology. ${ }^{27}$ However, in most instances, an understanding of the disease is helpful. For CLN2 disease, enzyme replacement therapy (ERT) is currently being applied in patients with promising results. ${ }^{28,29}$ This therapy is based on the insight that CLN2 is a soluble lysosomal enzyme, making it eligible for crosscorrection. Given the nature of CLN3 disease, we suspect that multi-modal treatment options will be needed to target all aspects of disease. Possibly, the development of the first treatment strategies will elucidate some new aspects of the pathophysiology, that will in turn allow to improve and/or develop new treatment strategies.

A doctor's main aim is to cure people. However: 'La médecine c'est guérir parfois, soulager souvent, consoler toujours' (medicin is to cure sometimes, to relieve often, to comfort always) is especially true for Batten disease. At present, the fact that we cannot cure our patients, strengthens our obligation to do everything we can to at least alleviate the burden of disease. To truly do so, we as doctors must realize that not only the patient, but (particularly) the family and others close to the patient suffer from the disease. Currently, these 'bystanders' are often not on our horizon. For example, we often do not see the siblings at all, and we are thus largely unaware of their difficulties 
in growing up with a sibling that won't grow up the same way due to Batten disease. Batten disease takes away normal life one bit at a time: from vision to skiing, from reading to communication. As a doctor, it may feel as if you have nothing to offer. I however want to plea that the opposite is true. Providing an environment where families can discuss the difficulties of life in all honesty, is the least we can and should offer. Acknowledging and having attention for the current fate these families face, does not halt the quest for a new fate at all. It is the other way around: only by honestly acknowledging the darker sides of life, will you be able to see the light.

\section{CONCLUDING REMARKS}

From 1826 until now, we have identified ${ }^{30,31}$, genetically delineated ${ }^{32}$ and clinically delineated (which this thesis aims to contribute to) Batten Disease. Once we understand the pathophysiological basis of disease and ultimately cure Batten disease, this thesis will represent the remnants of an old disease in an old time.

I hope that even then the deep phenotyping approach used throughout this thesis - which has allowed us to gain new insights by using the most out of often already existing data - will continue to be of use as an approach to understand (rare) disorders. This approach does not require fancy machinery, but it does require an open view. An open view helps us evade our blind spots and allows us to see the answers lying in front of us. 


\section{REFERENCES}

${ }^{1}$ Marshall FJ, de Blieck EA, Mink JW et al. A clinical rating scale for Batten disease: reliable and relevant for clinical trials. Neurology. 2005 Jul 26;65(2):275-9.

${ }^{2}$ Mink JW, Augustine EF, Adams HR, Marshall FJ, Kwon JM. Classification and natural history of the neuronal ceroid lipofuscinoses. J Child Neurol. 2013 Sep;28(9):1101-5.

${ }^{3}$ Diekman EF, de Koning TJ, Verhoeven-Duif NM, Rovers MM, van Hasselt PM. Survival and psychomotor development with early betaine treatment in patients with severe methylenetetrahydrofolate reductase deficiency. JAMA Neurol. 2014 Feb;71(2):188-94.

${ }^{4}$ Lang FM, Korner P, Harnett M, Karunakara A, Tifft CJ. The natural history of Type 1 infantile GM1 gangliosidosis: A literature-based meta-analysis. Mol Genet Metab. 2019 Dec 30. pii: S1096-7192(19)30840-6.

${ }^{5}$ Vandenbroucke JP. In defense of case reports and case series. Ann Intern Med. 2001 Feb 20;134(4):330-4.

${ }^{6}$ Munroe PB, Mitchison HM, O'Rawe AM et al. Spectrum of mutations in the Batten disease gene, CLN3. Am J Hum Genet. 1997 Aug;61(2):310-6.

${ }^{7}$ Kousi M, Lehesjoki AE, Mole SE. Update of the mutation spectrum and clinical correlations of over 360 mutations in eight genes that underlie the neuronal ceroid lipofuscinoses. Hum Mutat. 2012 Jan;33(1):42-63.

${ }^{8}$ Aberg L, Talling M, Härkönen T et al. Intermittent prednisolone and autoantibodies to GAD65 in juvenile neuronal ceroid lipofuscinosis. Neurology. 2008 Apr 1;70(14):1218-20.

${ }^{9}$ Ouseph MM, Kleinman ME, Wang QJ. Vision loss in juvenile neuronal ceroid lipofuscinosis (CLN3 disease). Ann N Y Acad Sci. 2016 May;1371(1):55-67.

${ }^{10}$ Bohra LI, Weizer JS, Lee AG, Lewis RA. Vision loss as the presenting sign in juvenile neuronal ceroid lipofuscinosis. J Neuroophthalmol. 2000 Jun;20(2):111-5.

${ }^{11}$ Collins J, Holder GE, Herbert H, Adams GG. Batten disease: features to facilitate early diagnosis. Br J Ophthalmol. 2006 Sep;90(9):1119-24.

${ }^{12}$ Wang F, Wang $\mathrm{H}$, Tuan HF et al. Next generation sequencing-based molecular diagnosis of retinitis pigmentosa: identification of a novel genotype-phenotype correlation and clinical refinements. Hum Genet. 2014 Mar;133(3):331-45. doi: 10.1007/s00439-013-1381-5.

${ }^{13}$ Ku CA, Hull S, Arno G et al. Detailed Clinical Phenotype and Molecular Genetic Findings in CLN3-Associated Isolated Retinal Degeneration. JAMA Ophthalmol. 2017 Jul 1;135(7):749760.

${ }^{14}$ Cooper JD. The neuronal ceroid lipofuscinoses: the same, but different? Biochem Soc Trans. 2010 Dec;38(6):1448-52.

${ }^{15}$ Nelvagal HR, Lange J, Takahashi K, Tarczyluk-Wells MA, Cooper JD. Pathomechanisms in the neuronal ceroid lipofuscinoses. Biochim Biophys Acta Mol Basis Dis. 2019 Oct 31:165570.

${ }^{16}$ Zeman W, Dyken P. Neuronal ceroid-lipofuscinosis (Batten's disease): relationship to amaurotic family idiocy? Pediatrics. 1969 Oct;44(4):570-83.

${ }^{17}$ Williams RE, Mole SE. New nomenclature and classification scheme for the neuronal ceroid lipofuscinoses. Neurology. 2012 Jul 10;79(2):183-91.

${ }^{18}$ Palmer DN. The relevance of the storage of subunit c of ATP synthase in different forms and models of Batten disease (NCLs). Biochim Biophys Acta. 2015 Oct;1852(10 Pt B):2287-91.

${ }^{19}$ Teinert J, Behne R, Wimmer M, Ebrahimi-Fakhari D. Novel insights into the clinical and molecular spectrum of congenital disorders of autophagy. J Inherit Metab Dis. 2020 Jan;43(1):51-62. 
${ }^{20}$ Stevanin G, Santorelli FM, Azzedine H, et al. Mutations in SPG11, encoding spatacsin, are a major cause of spastic paraplegia with thin corpus callosum. Nat Genet. 2007;39(3):366372.

${ }^{21}$ Palmieri M, Pal R, Nelvagal HR et al. mTORC1-independent TFEB activation via Akt inhibition promotes cellular clearance in neurodegenerative storage diseases. Nat Commun. 2017 Feb 6;8:14338.

${ }^{22}$ Lange J, Haslett LJ, Lloyd-Evans E et al. Glial cells are functionally impaired in juvenile neuronal ceroid lipofuscinosis and detrimental to neurons. Acta Neuropathol Commun. 2017 Oct 17;5(1):74.

${ }^{23}$ Pontikis CC, Cella CV, Parihar N et al. Late onset neurodegeneration in the Cln3-/- mouse model of juvenile neuronal ceroid lipofuscinosis is preceded by low level glial activation. Brain Res. 2004 Oct 15;1023(2):231-42.

${ }^{24}$ Ostergaard JR, Rasmussen TB, Mølgaard H. Cardiac involvement in juvenile neuronal ceroid lipofuscinosis (Batten disease). Neurology. 2011 Apr 5;76(14):1245-51.

${ }^{25}$ Cooper JD, Tarczyluk MA, Nelvagal HR. Towards a new understanding of NCL pathogenesis. Biochim Biophys Acta. 2015 Oct;1852(10 Pt B):2256-61.

${ }^{26}$ Siintola E, Topcu M, Aula $\mathrm{N}$ et al. The novel neuronal ceroid lipofuscinosis gene MFSD8 encodes a putative lysosomal transporter. Am J Hum Genet. 2007 Jul;81(1):136-46.

${ }^{27} \mathrm{Kim}$ J, Hu C, Moufawad El Achkar C et al. Patient-Customized Oligonucleotide Therapy for a Rare Genetic Disease. N Engl J Med. 2019 Oct 24;381(17):1644-1652.

${ }^{28}$ Markham A. Cerliponase Alfa: First Global Approval. Drugs. 2017 Jul;77(11):1247-1249.

${ }^{29}$ Schulz A, Ajayi T, Specchio N et al. Study of Intraventricular Cerliponase Alfa for CLN2 Disease. N Engl J Med. 2018 May 17;378(20):1898-1907.

${ }^{30}$ Stengel C, Beretning om et mærkeligt Sygdomstilfælde hos fire Sødskende i Nærheden af Røraas, Eyr Med. Tidskr. 1 (1826) 347-352.

${ }^{31}$ Batten F. Cerebral degeneration with symmetrical changes in the maculae in two members of a family, Trans. Ophthalmol. Soc. U. K. 23 (1903) 386-390.

${ }^{32}$ Isolation of a novel gene underlying Batten disease, CLN3. The International Batten Disease Consortium. Cell. 1995 Sep 22;82(6):949-57. 
| 9 

ADDENDUM 



\section{CHAPTER SUMMARY AND NEDERLANDSE SAMENVATTING}

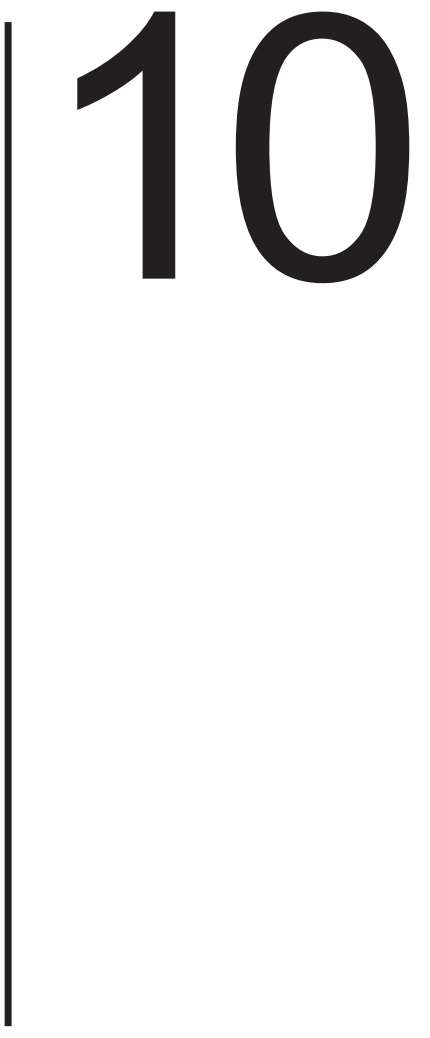




\section{SUMMARY}

The main aims of this thesis were: 1 ) to gain more insight in the early phase of Batten disease, particularly in the early ophthalmological and neurological signs, to improve early recognition, 2) to gain more insight in the following disease course, particularly the nuances in its phenotypic spectrum, and 3) to gain more insight in the cellular malfunctioning in Batten disease, particularly the storage material accumulation.

In Part 1, we focused on the first aim. In Chapter 2, we analyzed early neurocognitive functioning in CLN3 disease. We questioned the paradigm that onset of vision loss precedes onset of neurodegeneration by a few years in CLN3 disease. To analyze the timing of cognitive decline in CLN3 disease, we extracted age at onset of vision loss and age at onset of cognitive decline from cases described in literature, thus assembling a large cohort of literaturederived patients. We supplemented this cohort with data with regard to IQ test results performed around diagnosis, plus the early school history, from our 'own' cohort at the WKZ and the Bartiméus institute. To be able to distinguish the effects of vision loss from neurodegeneration, we analyzed classical CLN3 disease and protracted CLN3 disease separately, and added a control cohort of patients diagnosed with early-onset Stargardt disease (STGD1) - a retina-only disease with a similar age at onset- who were examined at diagnosis at the Bartiméus institute as well. We demonstrated that in classical CLN3 disease cognitive deficits are consistently present around the time of diagnosis: onset of cognitive decline (at a mean age of 6.8 years) actually paralleled onset of vision loss (at a mean age of 6.4 years) supported by an early decline in IQ scores (at diagnosis around 8 years of age, the IQ had already declined to 80 points). Moreover, in the Bartiméus cohort, early school history was already aberrant even before diagnosis in the majority of patients. In contrast, in earlyonset STGD1 patients, their vision loss did not impact their IQ scores and their school performance was uneventful.

We subsequently reasoned that, if cognitive functioning is already impaired early in the disease course of patients with classical CLN3 disease this may also hold true for motor function. To test this hypothesis, we analyzed motor function in CLN3 disease using the 6 minute walk test (6MWT) in Chapter 3. We administered the 6MWT repeatedly over a course of 7 years in a cohort of 15 CLN3 disease patients. To distinguish secondary effects of vision loss from direct effects resulting from neurodegeneration, two age-matched 
control cohorts also performed the 6MWT during one occasion: one cohort (14 patients) had isolated visual impairment, a second control cohort (12 patients) in addition had non-progressive neurologic impairment. In CLN3 disease, 6MWT results were already impaired from first testing around diagnosis (mean z-scores of -3.6 and -4.7 at 7 and 8 years of age, respectively) followed by a continuous decline, as expected with disease progression. In contrast, the isolated visual impairment group showed an upward trend of 6MWT results with age -similar to their healthy, sighted- peers. The control cohort individuals with additional neurologic impairments displayed a slightly decreased but stable 6MWT performance over time. Based on the results of Chapter $\mathbf{2}$ and 3, we therefore concluded that in (classical) CLN3 disease, neurodegeneration has its onset early in the disease course.

Although present from early in the disease course, the initial signs of neurologic involvement are subtle, especially when compared to the dramatically rapid and severe vision loss. Likely, these signs may be too subtle to be recognized by an ophthalmologist. In Chapter $\mathbf{4}$ we therefore assessed whether there are ophthalmological characteristics that allow differentiating patients with CLN3 disease from those with early-onset STGD1 disease. To do so, we performed an in-depth analysis of ophthalmological test results around diagnosis in 18 CLN3 disease patients and 20 early-onset STGD1 patients. We showed that CLN3 disease and early-onset STGD1 may seem to present similarly at first glance, but when you look closer, the disorders actually present quite differently. In CLN3 disease, the retina was more extensively affected (involving both cones and rods, as optic nerve associated layers) and more severely affected compared to early-onset STGD1. This led to several differentiating features. Three of these features most clearly aided to distinguish CLN3 disease: dramatically rapid loss of vision, severe color vision deficiency (both indicative of severe cone involvement) and absent or electronegative DA ERG responses (indicative of severe rod and inner retina involvement). As (untargeted) genetic analyses are not always available or desirable, we argued that the ability to recognize a (rare) disorder on clinical characteristics only is of ongoing relevance to avoid diagnostic delay, and to provide tailored counseling and guidance as early as possible.

In Part 2, we focused on the nuances in the phenotypic spectrum of CLN3 disease. In Chapter 5, we reported on three CLN3 disease patients with a mutation in the start codon of CLN3. Given the highly conserved start 
codon code among eukaryotic species, one would expect this mutation to be associated with the classical phenotype. However, all three patients displayed a milder phenotype than expected, with delayed cognitive decline and a particular absence of motor decline. In the absence of reliable functional assays, we could only speculate on the mechanisms of residual CLN3 protein activity. We hypothesized that alternative transcription could occur, either from an alternative downstream AUG codon (possibly already naturally present in alternative CLN3 transcripts) or even from the mutated non-AUG codon itself. Future research will have to elucidate the exact mechanisms behind this, and whether this residual CLN3 protein synthesis could even be enhanced, similar to what is being investigated in nonsense codon read through therapies.

At the very mild end of the phenotypic spectrum of CLN3 disease, recent research has suggested that some mutations in CLN3 may be exclusively associated with a retina-only phenotype. Thus, these patients may never develop any neurodegeneration. In Chapter 6 however, we report on a man, homozygous for one of these 'retina-only' mutations (the R405W mutation), who did develop adult-onset neurologic decline. He did not suffer from a parkinsonism movement disorder as characteristic for classical-protracted CLN3 disease, but he rather suffered from a spastic movement disorder. Extensive examinations did not reveal a different cause for his complaints. We therefore concluded that the neurologic decline was due to (atypical) CLN3 disease, particularly since he also suffered from increasing cognitive impairments. Thus, prudency is warranted when counseling these patients, as CLN3-associated retinal degeneration may in some cases include neurological deterioration.

Part 1 and Part 2 make clear that it is not only difficult to recognize CLN3 disease patients, but it is also difficult to counsel them correctly. From classical up to and including protracted CLN3 disease, initial disease presentation may be very similar, but the following disease course may significantly differ from childhood onset to adult-onset neurologic decline. CLN3-associated (isolated) retinal degeneration presents differently from classical-protracted CLN3 disease, but these patients may or may not develop neurological decline. There is a clinical need for a 'marker' that allows to classify disease severity and thus to predict the following disease course. To address this, we focused on the storage material accumulation in Batten disease in Part 3. Within the 
lymphocytes of CLN3 disease patients, the storage material accumulation is displayed as so-called vacuoles, that are visible on light microscopy. In Chapter 6, we hypothesized that the degree of lymphocyte vacuolization may be less abundant in milder forms of the disease. In Chapter 7, we assessed whether quantifying the degree of lymphocyte vacuolization could facilitate its use as a diagnostic marker for CLN3 disease, and even as an index of disease severity. Over the course of 7 years, we collected 67 peripheral blood samples from a total of 19 patients comprising the complete CLN3 disease spectrum - from classical CLN3 disease to CLN3-associated retinal degeneration. Lymphocyte vacuolization was quantified using a manual and an automated approach. In the manual approach, both the percentage of vacuolated lymphocytes and the number of vacuoles per affected lymphocyte correlated with disease severity. In the automated approach, a similar correlation was seen, supplemented with insight in the distribution of the storage material among the different lymphocyte subsets: In classical and protracted CLN3 disease, LAMP-1 expression was mainly confined to the CD4 and CD8 compartment (T-cells) while in CLN3-associated retinal degeneration, LAMP-1 expression was predominantly confined to the CD20 compartment (B-cells). Although we do not yet understand why the storage material accumulation is distributed so differently at the mild end of the phenotypic spectrum, it currently already provides a valuable way to diagnose CLN3 disease and simultaneously classify disease severity.

CLN3 belongs to a group of proteins that, if deficient, all cause an NCL with shared clinical and pathological characteristics. The shared disease characteristics suggest that the CLN proteins function together. However, up to now, the function of most CLN proteins has remained enigmatic. In Chapter $\mathbf{8}$ we hypothesized that the CLN proteins might function in the turnover of either saposins A and D (CLN1, CLN4, CLN10) or subunit c (other CLN proteins). This would explain the correlation between the degree of residual CLN3 activity and the degree of storage material accumulation as proposed in Chapter 7, and it would explain why the NCLs accumulate these proteins specifically, rather than a heterogeneous mixture of storage material, if this would only be a byproduct of general lysosomal malfunctioning.

Taken together, this thesis provides insights in Batten disease which we gained by 'looking beyond our blind spots'. In Part 1, we provided added insight in the early phase of the disease, which hopefully improves early recognition and 
better tailored counseling. We increasingly learn that the following disease course can vary dramatically between patients. In Part 2, we provided some new insights in the phenotypic spectrum of Batten disease. Finally, in Part 3, we aimed to connect disease with pathophysiology, focused on the storage material accumulation, as we proposed that this 'old' marker deserves to be looked at with new eyes. Ultimately, we hope that the work done in this thesis will help to pave the way towards treatment for this devastating disease. 


\section{NEDERLANDSTALIGE SAMENVATTING}

De ziekte van Batten is een metabole ziekte, oftewel een stofwisselingsziekte. Bij de ziekte van Batten raakt de functie van het lysosoom verstoord. Het lysosoom stond lang bekend als de 'afvalverwerker' van de lichaamscel, maar inmiddels weten we dat het lysosoom daarnaast ook een centrale rol speelt bij allerlei andere processen in de cel. Hoe de lysosomale functie bij de ziekte van Batten precies verstoord raakt, is nog niet goed bekend. Het gebrek aan begrip van wat er precies gebeurt bij de ziekte van Batten, heeft het ontwikkelen van een behandeling tot nu erg moeilijk gemaakt. In dit proefschrift vergeleken we het gebrek aan begrip met de blinde vlek: letterlijk en figuurlijk een centraal onderdeel in de ziekte van Batten.

De eerste twee delen van dit proefschrift richten zich met name op het begrijpen van de klinische kant van de ziekte van Batten: wat zien we precies bij de patiënt? In het derde deel van dit proefschrift proberen we wat we zien bij de patiënt te verbinden met wat we zichtbaar kunnen maken in de cel, waarbij we ons met name richten op het kenmerkende stapelingsmateriaal bij de ziekte van Batten.

\section{DEEL I - VROEGE HERKENNING VAN DE ZIEKTE VAN BATTEN}

Hoewel er nog geen behandeling is die de ziekte kan vertragen of stoppen, is het toch belangrijk de ziekte van Batten tijdig te herkennen. Hierdoor kan meteen de juiste begeleiding worden gegeven - die anders is dan bij een kind met een geïsoleerde netvliesziekte. Tijdige herkenning van de ziekte van Batten is echter moeilijk omdat de ziekte in het begin juist zo op een geïsoleerde netvliesziekte lijkt - dat was althans de gangbare gedachte. Men ging er van uit dat bij de ziekte van Batten de achteruitgang van het netvlies pas na enkele jaren gevolgd wordt door achteruitgang van de hersenen. Wij zagen echter dat patiënten vaak al vroeg leer- en/of gedragsproblemen hadden, die vervolgens - logischerwijs - werden toegeschreven aan het slechte zicht. In Hoofdstuk 2 vroegen we ons daarom af of de achteruitgang van de hersenen misschien toch eerder begint dan tot nu toe werd gedacht. Om dit te onderzoeken verzamelden wij publicaties van patiënten met Batten, die we opdeelden in patiënten met de klassieke vorm en de protracted vorm deze laatste vorm staat erom bekend dat hersenachteruitgang soms pas in de volwassenheid begint - en analyseerden de IQ scores ten tijde van diagnose. Dit vulden wij aan met 'onze' eigen patiënten, waarbij wij de bassischoolhistorie voor diagnose en de resultaten van het IQ onderzoek (bij Bartiméus standaard 
uitgevoerd) rond diagnose verzamelden. Deze groep vergeleken wij met kinderen met early-onset Stargardt (STGD1): de ziekte waarmee Batten in het begin vaak wordt verward, maar die alleen het netvlies aantast. Onze vermoedens bleken te kloppen: Bij (de klassieke vorm van) Batten was het IQ rond diagnose al verlaagd (op de leeftijd van 8 jaar was het IQ rond de 80) en had de meerderheid al voor diagnose leerondersteuning nodig gehad. Dit kwam niet door het slechte zicht: patiënten met protracted Batten en earlyonset STGD1 zagen net zo slecht als kinderen met de klassieke vorm van Batten, maar hadden een normale IQ score en schoolhistorie.

Wij vermoedden, dat als het leren al vroeg is aangedaan als uiting van hersenachteruitgang, dit ook wel eens voor de motoriek zou kunnen gelden. Om dit te onderzoeken verzamelden wij in Hoofdstuk 3 alle gegevens van de 6-minuten looptest (6MWT) die standaard 2 keer per jaar op onze multidisciplinaire NCL poli wordt uitgevoerd. De 6MWT werd bij veel andere aandoeningen al gebruikt als een maat voor algeheel functioneren en achteruitgang, maar nog niet bij slechtzienden, inclusief de ziekte van Batten. Gedurende 7 jaar hebben wij deze 6MWT uitgevoerd bij 15 patiënten. Vanzelfsprekend moest ook hierbij onderscheid kunnen worden gemaakt tussen slechtziendheid en hersenachteruitgang. Daarom hebben wij een groep kinderen van de Bartiméusschool in Zeist de 6MWT ook laten uitvoeren. Allen hadden een visuele beperking, een deel van hen had geen verdere problemen, een deel van hen had ook (niet-progressieve, dus stabiele) neurologische problemen. De resultaten van dit onderzoek lieten zien dat bij de ziekte van Batten, de 6MWT resultaten al vanaf het begin duidelijk verminderd waren ten opzichte van de referentiewaarden, zoals vastgesteld voor gezonde, goedziende leeftijdsgenoten. Dit kon wederom niet verklaard worden door het slechte zicht, omdat ook de slechtziende leeftijdsgenoten goed liepen. De kinderen met bijkomende neurologische problemen liepen minder ver dan hun gezonde, slechtziende leeftijdsgenoten, maar interessant genoeg, nog altijd beter dan de kinderen met de ziekte van Batten. Dus, Hoofdstuk 2 en Hoofdstuk 3 laten zien dat de neurologische achteruitgang bij (klassiek) Batten al vroeg in het ziektebeloop begint.

Hoewel Hoofdstuk $\mathbf{2}$ en $\mathbf{3}$ overtuigend laten zien dat neurologische achteruitgang al vroeg begint, is dit bij aanvang hiervan nog steeds subtiel. De achteruitgang van het zicht staat dan op de voorgrond, wat dan ook de reden is dat deze kinderen (vaak) eerst bij de oogarts terecht komen. Voor de 
oogarts is het moeilijk de ziekte van Batten te herkennen: de achteruitgang van het zicht - door achteruitgang van het netvlies - lijkt in dit stadium nog erg op de ziekte van Stargardt, en bovendien ziet de gemiddelde oogarts (veel) te weinig patiënten met Batten om deze patiënten op ervaring te kunnen herkennen. In Hoofdstuk 4 wilden wij daarom onderzoeken of we in deze vroege fase onderscheidende oogheelkundige kenmerken konden vinden die de oogarts helpen de ziekte van Batten te herkennen. Om dit te doen vergeleken wij de resultaten van het oogheelkundige onderzoek rond diagnose van 18 Batten patiënten met 20 early-onset Stargardt patiënten. Wij vonden dat, hoewel de beide ziekten op het eerste oog op elkaar lijken, er wel degelijk onderscheidende verschillen te zien zijn als je goed inzoomt: zo zien de beide groepen initieel vergelijkbaar (slecht), maar gaat het zicht bij Batten veel harder achteruit: dit verschil in snelheid is al in het 1e jaar merkbaar. Daarnaast bleek de ernst van het kleurenzien ook vaak een duidelijk onderscheidende factor, en wel zodanig dat we konden stellen dat als het kleurenzien normaal is (bij een kind in de basisschoolleeftijd met een macula atrofie), er sprake was van Stargardt, maar als het kleurenzien ernstig gestoord is, er sprake was van de ziekte van Batten. Beide kenmerken - snelle achteruitgang van het zicht en ernstige kleurenzienstoornis - wijzen op vroege en ernstige schade aan de kegeltjes. Het duidelijkst onderscheidende onderzoek was het electrofysiologisch onderzoek: het ERG. De donker geadapteerde responsies van het ERG waren bij Batten patiënten allemaal electronegatief of afwezig, terwijl deze bij de Stargardt patiënten hoogstens mild aangedaan waren. Deze ERG resultaten wijzen op een vroege en ernstige betrokkenheid van de staafjes (terwijl deze bij Stargardt niet of nauwelijks aangedaan waren) en het binnenste netvlies (terwijl dit bij Stargardt niet betrokken was). Dus, bij de ziekte van Batten was het netvlies al vroeg uitgebreider en ernstiger aangedaan, wat zich uit in verschillende onderscheidende kenmerken waarop de oogarts de ziekte van Batten kan herkennen.

\section{DEEL II - HET FENOTYPISCH SPECTRUM VAN DE ZIEKTE VAN BATTEN}

Wij wilden niet alleen beter inzoomen op de vroege fase van de ziekte, maar ook op het ziektebeloop als geheel. Wij vermoedden dat hierop beter inzoomen meer inzicht zou geven in de ziekte. Inderdaad geven Hoofdstuk $\mathbf{5}$ en $\mathbf{6}$ belangrijke nuances in het ziektebeloop aan. In Hoofdstuk $\mathbf{5}$ beschrijven wij dat wij een patiënt zagen met één keer de standaard 1 kb deletie en één keer een mutatie (ziekte-veroorzakende DNA verandering) helemaal in het begin van het CLN3 gen. Dit begin is voor elk gen hetzelfde, vandaar dat 
mutaties hierin over het algemeen als ernstig worden ingeschat. Zo ook bij deze patiënt. Echter, gedurende de jaren bleef hij neurologisch vrijwel stabiel. Hierdoor betwijfelden wij of de zogenaamde start codon mutatie wel een ernstige mutatie was. Gezien de moeilijkheden om onderzoek te doen naar het CLN3 eiwit zelf, konden we niet eenvoudig meten of en hoeveel (werkzaam) CLN3 eiwit er was. Daarom besloten we naar andere patiënten te zoeken. We vonden nog twee patiënten met precies hetzelfde genotype die ook een vertraagd beeld lieten zien, waarbij een van hen zelfs de reguliere middelbare school heeft afgemaakt. Dit sterkte ons in de hypothese dat deze start codon mutatie mogelijk nog wat rest CLN3 productie lijkt te geven.

Hoewel we al langer weten dat sommige mutaties een vertraagd (neurologisch) beeld kunnen geven, was de consensus dat de ziekte van Batten altijd, vroeg of laat, leidt tot achteruitgang van de hersenen. Recent onderzoek heeft echter laten zien dat bepaalde 'extreem milde' mutaties in CLN3 slechts tot een netvliesziekte zouden leiden. Bij dit beeld, genoemd 'CLN3 associated isolated retinal degeneration', zouden de hersenen dus helemaal niet betrokken zijn. In Hoofdstuk 6 laten wij echter zien dat isolated beter tussen haakjes geplaatst kan worden. Wij beschrijven een patiënt met 2 van zulke 'extreem milde' mutaties in CLN3 die wel degelijk neurologisch achteruitging. In Hoofdstuk 6 pleiten wij daarom voor voorzichtigheid, voordat deze patiëntengroep een retina-only ziekte wordt 'beloofd'.

\section{DEEL III - HET STAPELINGSMATERIAAL IN DE ZIEKTE VAN BATTEN HERZIEN}

Deel I en II van dit proefschrift maken duidelijk hoe ingewikkeld de ziekte van Batten is. Het is ingewikkeld om de ziekte van Batten te herkennen, maar ook om vervolgens in te schatten hoe het ziektebeloop zal zijn. Klassiek en protracted Batten lijken bij presentatie - qua netvliesachteruitgang - sterk op elkaar maar de timing en/of snelheid van de neurologische achteruitgang verschilt, terwijl bij CLN3-associated retinal degeneration de een wel, en de ander geen neurologische verschijnselen zal krijgen. Wat tot nu toe ontbrak was een soort 'marker' die richting geeft hoe het ziektebeloop zal zijn. In Hoofdstuk $\mathbf{7}$ beschrijven wij hoe wij, door met nieuwe ogen naar een oud kenmerk van de ziekte van Batten te kijken, namelijk de gevacuoliseerde lymfocyten, een marker hebben ontwikkeld voor diagnose en inschatten van ziekte-ernst. 
Ondanks de eerste beschrijving van de ziekte bijna 200 jaar geleden, blijft Batten nog altijd een raadselachtige ziekte, waarbij de functie van de meeste CLN eiwitten nog grotendeels onbekend is. In Hoofdstuk $\mathbf{7}$ doken we al in het stapelingsmateriaal, een typisch verschijnsel van de ziekte van Batten. In Hoofdstuk 8 lichtten we onze hypothese toe dat de samenstelling van dit stapelingsmateriaal mogelijk meer inzicht zou kunnen geven in de functie van de CLN eiwitten.

Alles samenvattend beoogt dit proefschrift meer inzicht te geven in de ziekte van Batten, met name door bepaalde blinde vlekken inzichtelijk te maken en weg te nemen. Uiteindelijk hopen we volledig inzicht te krijgen in de ziekte van Batten, om zo deze verwoestende ziekte te kunnen genezen. 


\section{LIST OF ABBREVIATIONS}

$1 \mathrm{~kb}$ deletion = 1 kilo base deletion = in the context of CLN3 disease a 966 base pair deletion spanning exon 7 and 8 present in the majority of CLN3 disease patients 6MWT = six minute walk test ABCA4 = ATP-binding cassette, sub-family A (ABC1), member 4 ATP13A2 = ATPase cation transporting 13A2

$\mathrm{BCVA}=$ best corrected visual acuity CLN3 = ceroid lipofuscin neuronal 3 (gene)

CLN3 = ceroid lipofuscin neuronal 3 (protein)

CLN3 disease $=$ neuronal ceroid lipofuscinosis type 3 (Batten disease)

CTSD = cathepsin D

$\mathrm{CTSF}=$ cathepsin $\mathrm{F}$

CVP = curvilinear profile

$\mathrm{DA}=$ dark adapted

DNAJC5 = DnaJ heat shock protein family (Hsp40) member C5

DTL = Dawson-Trick-Litzkow

$\mathrm{EM}=$ electron microscopy

IEM = immuno-electron microscopy

$\mathrm{ERG}=$ electroretinogram

ERT = enzyme replacement therapy

FACS = fluorescence automated cell sorting

$\mathrm{FAF}=$ fundus autofluorescence

ffERG = full-field electroretinogram

FPP = fingerprint profile

GROD = granular osmiophilic deposit

$\mathrm{GRN}=$ granulin

HRR $=$ Hardy Rand Rittler

IEM = inborn error of metabolism

ISCEV = International Society for Clinical Electrophysiology of Vision

$\mathrm{IQ}=$ intelligence quotient

$\mathrm{JNCL}=$ juvenile neuronal ceroid lipofuscinosis

KCTD7 = potassium channel tetramerization domain containing 7

$\mathrm{LA}=$ light adapted

LAMP-1= lysosomal associated membrane protein 1 (also: CD107a)

LAMP-2= lysosomal associated membrane protein 2 (also: CD107b)

LDAO = lauryldimethylamine- $\mathrm{N}$-oxide

$\mathrm{LM}=$ light microscopy

LSD = lysosomal storage disorder 
MFSD8 = major facilitator superfamily domain containing 8

$\mathrm{NCL}=$ neuronal ceroid lipofuscinosis

$\mathrm{NFL}=$ nerve fiber layer

OCT = optical coherence tomography

$\mathrm{OD}=$ oculus dexter (right eye)

OMIM = Online Mendelian Inheritance in Man

OS = oculus sinister (left eye)

$\mathrm{PIQ}=$ performal intelligence quotient

PPT1 = palmitoyl protein thioesterase 1 (CLN1)

Saposin = sphingolipid activator protein

STGD1 = Stargardt disease

TFEB $=$ transcription factor $E B$

$\mathrm{TIQ}=$ total intelligence quotient

TPP1 = tripeptidyl peptidase 1 (CLN2)

$R G=$ red-green (in the context of color vision deficiency)

$\mathrm{RLB}=$ rectilinear body

$\mathrm{RPE}=$ retinal pigment epithelium

UBDRS = Unified Batten Disease Rating Scale

$\mathrm{VA}=$ visual acuity

$\mathrm{VIQ}=$ verbal intelligence quotient

VOD = visual acuity of the right eye

VODS = vision of both eyes

VOS = visual acuity of the left eye

$\mathrm{WHO}=$ World Health Organization

WKZ = Wilhelmina Children's Hospital (Wilhelmina Kinderziekenhuis) 


\section{LIST OF PUBLICATIONS}

\section{IN THIS THESIS}

Kuper WFE, van Alfen C, Rigterink RH, Fuchs SA, van Genderen MM, van Hasselt PM. Timing of cognitive decline in CLN3 disease. J Inherit Metab Dis. 2018 Mar;41(2):257-261.

Kuper WFE, van Alfen C, van Eck L, Huijgen BCH, Nieuwenhuis EES, van Brussel $M$, van Hasselt PM. Motor function impairment is an early sign of CLN3 disease. Neurology. 2019 Jul 16;93(3):e293-e297.

Kuper WFE, Talsma HE, van Schooneveld MJ, Pott JWR, Huijgen $\mathrm{BCH}$, de Wit GC, van Hasselt $\mathrm{PM}^{*}$, van Genderen $\mathrm{MM}^{*}$. Differentiating ophthalmological characteristics of CLN3 disease (Batten disease). Submitted. ${ }^{*}$ contributed equally

Kuper WFE, van Alfen C, van Eck L, de Man SA, Willemsen MH, van Gassen $\mathrm{KLI}$, Losekoot M, van Hasselt PM. The c.1A>C start codon mutation in CLN3 is associated with a protracted disease course. JIMD Rep. 2020 Feb 7;52(1):23-27.

Kuper WFE, van Alfen C, van Eck L, van den Broek BTA, Huisman A, van Genderen MM, van Hasselt PM. A case of unexpected adult-onset neurologic decline in CLN3-associated retinal degeneration. JAMA Ophthalmol. 2017 Dec 1;135(12):1451-1453.

Kuper WFE, Oostendorp M, van den Broek BTA, van Veghel K, Nonkes LJP, Nieuwenhuis EES, Fuchs SA, Veenendaal T, Klumperman J, Huisman A, Nierkens $S^{\star}$, van Hasselt $\mathrm{PM}^{\star}$. Quantifying lymphocyte vacuolization serves as a measure of CLN3 disease severity. Revised for JIMD reports. * contributed equally

Kuper WFE*, Rehmann $\mathrm{H}^{*}$, van Hasselt PM. Storage material accumulation in the neuronal ceroid lipofuscinoses revisited. In progress. ${ }^{*}$ contributed equally 


\section{OTHER}

Gomez-Giro G, Arias-Fuenzalida J, Jarazo J, Zeuschner D, Ali M, Possemis N, Bolognin S, Halder R, Jäger C, Kuper WFE, van Hasselt PM, Zaehres H, del Sol A, van der Putten H, Schöler HR, Schwamborn JC. Synapse alterations precede neuronal damage and storage pathology in a human cerebral organoid model of CLN3-Juvenile Neuronal Ceroid Lipofuscinosis. Acta Neuropathol Commun. 2019 Dec 30;7(1):222.

Milev $M^{\star}$, Graziano $C^{\star}$, Karall D*, Kuper WFE*, Al-Deri N, Cordelli DM, Haack TB, Danhauser K, luso A, Palombo F, Pippucci T, Prokisch H, Saint-Dic D, Seri M, Stanga D, Cenacchi G, van Gassen KLI, Zschocke J, Fauth C, Mayr JA, Sacher M, van Hasselt PM. Bi-allelic mutations in TRAPPC2L result in a neurodevelopmental disorder and have an impact on RAB11 in fibroblasts. $J$ Med Genet. 2018 Nov;55(11):753-764.

* contributed equally

de Sain-van der Velden MGM*, Kuper WFE*, Kuijper M-A, van Kats LAT, Prinsen HCMT, Balemans ACJ, Visser G, van Gassen KLI, van Hasselt PM. Beneficial effect of BH4 treatment in a 15 year old boy with biallelic mutations in DNAJC12. JIMD Rep. 2018;42:99-103.

* contributed equally 


\section{DANKWOORD}

Ik schrijf dit dankwoord in april 2020, op het moment dat de COVID-19 pandemie ook Nederland in zijn greep heeft. Op het eerste oog lijkt deze ziekte weinig parallellen te hebben met een metabole ziekte. Waar er bij COVID-19 sprake is van een acute infectieziekte, die velen van ons bedreigt, is er bij een metabole ziekte over het algemeen sprake van een chronische ziekte, die maar enkelen treft, of lijkt te treffen. Ik zie echter vooral parallellen. In het geval van COVID-19 zijn velen vooral zo bezorgd en/of kwetsbaar omdat een ziekte ons bedreigt, waar nog geen medicijn voor is. Voor families met een kind met een metabole ziekte, zoals Batten, is dit - vaak al jaren - hun dagelijkse realiteit. Ik hoop dat als wij straks op de COVID-19 pandemie terugkijken, wij ons extra bewust zijn van de allesoverheersende dreiging van een onbehandelbare ziekte.

Het onderzoek dat wordt beschreven in dit proefschrift beoogt voor de ziekte van Batten, een van de vele onbehandelbare metabole ziekten, perspectief te bieden op een behandeling. Dit was niet gelukt zonder de hulp van velen, waarvan ik er hieronder enkelen wil noemen.

Allereerst wil ik alle Batten patiënten en hun families, met name hun ouders, bedanken. Gedurende de jaren dat ik - eerst als student, later als arts betrokken was bij de NCL poli en bij het onderzoek, hebben jullie mij toegelaten in vaak moeilijke momenten van jullie leven. Ik heb grote bewondering voor de moed en kracht waarmee jullie dit oneerlijke lot aangaan. Ik blijf mij graag inzetten om dit lot op een gegeven moment te kunnen keren.

Dit promotieonderzoek heeft kunnen plaatsvinden dankzij de financiële steun van het Bartiméus Fonds en de stichting Beat Batten. Ik wil hier met name Ronald en Addy noemen. De afgelopen jaren hebben wij intensief contact gehad. Ik bewonder de energie waarmee jullie niet alleen voor je eigen kind, maar voor alle kinderen met Batten het verschil willen maken.

De leden van de beoordelingscommissie, prof. dr. Judith Klumperman, prof. dr. Jeffrey Beekman, prof. dr. Nanda Verhoeven-Duif, prof. dr. Carel Hoyng en prof. dr. Kees Braun wil ik bedanken voor hun bereidheid dit proefschrift te beoordelen. Ook dank aan dr. Femke van Wijk en prof. dr. Ludo van der Pol voor het zitting nemen in de promotiecommissie. 
Mijn promotoren, Edward en Mies. Een promotie-traject gaat behalve over de inhoud, vooral over persoonlijke ontwikkeling. Edward, jij bezit als geen ander de gave om te roeren in de ziel. Dank voor onze waardevolle gesprekken. Mies, ik ben onder de indruk van jouw staat van dienst op het gebied van de oogheelkundige diagnostiek bij zeldzame ziekten. Je houdt altijd oog voor het belang van de patiënt. Dank voor je prettige begeleiding.

Mijn copromotor, Peter. Eigenlijk verdien je een pagina in dit dankwoord, maar omdat jij mij leerde dat bij schrijven geldt 'less is more', zal ik proberen dat ook hier toe te passen. Bijna 9 (!) jaar geleden mailde ik jou voor het eerst met de vraag of ik op de afdeling metabole ziekten onderzoek zou kunnen doen. Ik kan me herinneren dat het gelijk klikte. Je gaf me een A4tje mee met een lijstje metabole ziekten waar "we nog veel te weinig van begrijpen". Een van die ziektes was de ziekte van Batten. Ik had daarvoor nog nooit van deze ziekte gehoord, maar na een interview met ouders gelezen te hebben wist ik: hier wil ik mij mee bezig houden. Dat deze nieuwsgierigheid van toen, nu is uitgemond in een proefschrift, is voor een belangrijk deel aan jou te danken. Ik hoop dat we nog lange tijd samen zullen werken! Annika, dank voor de waardevolle coaching.

Het bijzondere aan mijn promotietraject was de nauwe samenhang tussen onderzoek en kliniek. Alle leden van het NCL expertisecentrum ben ik dankbaar voor hun rol en input. Claudia, jij bent de drijvende kracht achter het NCL expertisecentrum. Jouw klinische expertise is ongeëvenaard. Dankjewel voor alle hulp en samenwerking. Margreet, dank voor je ondersteuning van het NCL expertisecentrum. De overige leden van het expertisecentrum, met name Linda: hoewel je altijd zegt absoluut geen onderzoeker te zijn, komen er altijd veel (onderzoeks)ideeën en zelfs data uit jouw koker. Misschien ben je toch meer een onderzoeker dan je dacht. Cis en Paula, jullie hebben veel werk gestoken in een mooi onderzoeksplan waarmee we onder leiding van Yvonne het gedrag van kinderen met Batten beter leren begrijpen en hen vooral ook beter leren begeleiden. Dank voor jullie werk. In het WKZ zou ik niet zonder de ondersteuning van Sanna en Ellen hebben gekund. Saskia, dank voor de gezelligheid op onze 'tijdelijke' kamer.

Verschillende hoofdstukken waren niet tot stand gekomen zonder de essentiële bijdragen van de volgende personen. Ik noemde al even Linda: dankzij jouw onvermoeibare motorische testen tijdens de NCL poli, hebben 
we een heel mooi artikel in Neurology kunnen publiceren. Marco, dank voor je input vanuit de inspanningsfysiologie en dat je me in contact bracht met Barbara. Barbara, dankzij jouw analyses is ons onderbuikgevoel dat de motoriek (ook) al vroeg is aangedaan, statistisch solide bevestigd. Herman en Gerard: dankzij jullie inspanningen om met name de ERG resultaten duidelijk in beeld te brengen is hoofdstuk 4 zo duidelijk geworden. Mary, Jan Willem en Mies: dank voor jullie beoordelingen van de OCT scans. Stella, Monique, Koen en Marjolein: dank voor de samenwerking waarmee we in hoofdstuk 5 een nieuwe genotype-fenotype correlatie konden beschrijven. Dank aan de ouders en de zus van (een van) de patiënten van dit hoofdstuk. Het onderzoek beschreven in hoofdstuk 7 zou ook eigenlijk zijn eigen pagina verdienen. Al voordat ik begon, heb jij, Marlies, samen met Albert en Peter een belangrijk deel van de dataverzameling gedaan. Ook dank aan de analisten, met name Evertine: mede dankzij jullie bereidheid elk uitstrijkje drie (!) keer na te tellen is dit onderzoek zo robuust geworden als het nu is. Stefan en met name Karin: dank voor al jullie werk met de FACS analyse. De assay is nu slechts een 'vakje' op het labformulier in HiX, maar ik ben niet vergeten hoe veel werk het was om deze analyse tot een echte diagnostische kleuring te maken. Brigitte, dankjewel: sowieso voor de analyses in dit hoofdstuk, maar vooral ook voor alle gezelligheid. Lourens, leuk dat je hierbij bent aangehaakt, en ik ben benieuwd wat het vacuolen-algoritme nog brengt! Judith en Tineke: dank voor jullie werk met de elektronenmicroscopie, dit heeft een extra laag van begrip aan het onderzoek toegevoegd. Holger: dankzij jou hebben we in hoofdstuk 8 een mooie, solide hypothese kunnen neerzetten hoe wij denken dat de pathofysiologie van Batten in elkaar zit. Ik hoop dat we dit in vervolgonderzoek kunnen gaan toetsen in de praktijk. Ten slotte, Klaas, bedankt voor het sparren over hoofdstuk, 9, de discussie, met name over de rol van dokters bij een (nog) onbehandelbare ziekte.

De studenten die in de afgelopen jaren aan het onderzoek hebben meegewerkt: Sanne, Emma, Nina en Bram: dank voor jullie werk. Soumaya: je bent nog niet zo lang begonnen met je onderzoek, maar ik zie hoe leuk je het vindt, en dat is volgens mij de belangrijkste motor voor het doen van onderzoek.

Ondanks alle samenwerking, is promoveren relatief eenzaam. Gelukkig zijn daar andere promovendi om elkaar te steunen. Met name de koffiebitches kan ik hier niet onvermeld laten. Lieve Birgit, Carmen en Mimount, zonder onze koffie-bitchmomentjes en sushi-bitch momentjes waren de afgelopen 
jaren nooit zo leuk geweest. Dank voor jullie steun en luisterend oor, ook nu we onze koffiebitch-momentjes noodgedwongen digitaal doen. Birgit, wij sleepten elkaar al door de eerste stappen van het promotie-traject heen toen we nog op de balzaal zaten met Ineke en Laura. We waren onze tijd ver vooruit met de zeeën van ruimte die we hadden, waarbij we zelfs de prullenbak op anderhalve meter afstand hielden.

Anne en Petra: sinds onze $1^{\mathrm{e}}$ geneeskundewerkgroep trekken we al samen op, en ik hoop dat we dat nog lang zullen blijven doen! Mijn andere vriendinnen en vrienden: dank voor alle interesse, en juist ook voor alle afleiding. Lieve Blanca, mijn paranimf, dank dat je er voor me bent.

Mijn schoonfamilie: een schoonfamilie is het ultieme relatiegeschenk gelukkig heb ik geluk gehad met jullie. Frouwien, Huub, Angela, Rob en Ilona: dank voor jullie betrokkenheid. Mijn broertjes, Eelko en Joppe, met zijn drieën vormen wij een goed team. Unaniem weten wij altijd precies wat er van onze eigen app-groep wel (niets) en niet (alles) gedeeld kan worden in de gezins-appgroep. Lara, jij bent uitstekend geïntegreerd in de familie. Waar wij dachten dat onze Sinterklaas-gedichten inmiddels niet scherper konden, heb jij met de Sinterklaas-rebussen een hele nieuwe dimensie aan de avond toegevoegd. Mijn ouders, papa en mama, zonder jullie was dit proefschrift er sowieso niet geweest. Jullie hebben altijd onvoorwaardelijk veel vertrouwen in mij. Papa, dank dat jij mij al vroeg in mijn promotie wees op wat belangrijk is: "de kaft, de rest lezen ze toch niet". Ik ben al vroeg na gaan denken over de kaft en titel, en behalve dat ik met allebei heel blij ben, denk ik ook dat het nadenken over het grotere plaatje het onderzoek nog beter heeft gemaakt. Ik denk overigens dat men ook wel het dankwoord leest. Mocht je als lezer hier beland zijn, en je naam missen: ook jij heel erg bedankt.

Roel, jij bent mijn rots in de branding. Ik ben dankbaar dat ik met jou mijn leven mag delen. 


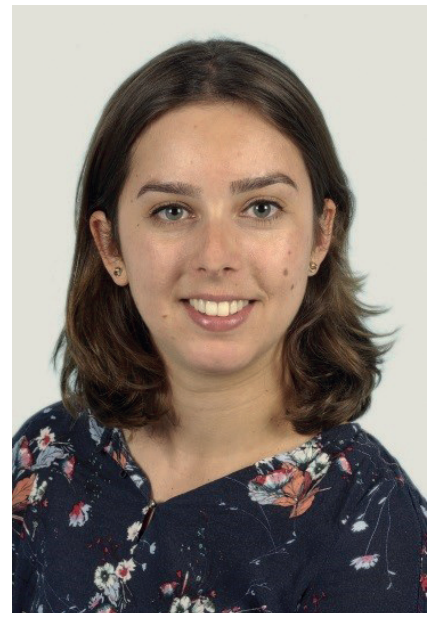

\section{CURRICULUM VITAE (NEDERLANDS)}

Willemijn Kuper werd geboren op 12 december 1991 te Nijmegen. Gedurende haar jeugd woonde ze met haar ouders (Fred en Dineke) en broertjes ${ }^{1}$ (Eelko en Joppe) in Wijchen. De middelbare school doorliep ze aan het Stedelijk Gymnasium Nijmegen, waar ze in 2010 het eindexamen behaalde. In datzelfde jaar startte ze haar studie Geneeskunde in Utrecht. Al vroeg in de studie brachten haar behoefte aan diepgang en interesse in onderzoek doen haar bij de afdeling metabole ziekten, waar dr. van Hasselt "nog wel een lijstje ziektes had waar we nog veel te weinig van begrijpen". Een van die ziektes was de ziekte van Batten. Als onderdeel van het honoursprogramma (bachelor en master) werkte ze aan het beter in beeld brengen van het natuurlijk beloop van deze ziekte - in het bijzonder de timing van de cognitieve achteruitgang - wat uiteindelijk heeft geleid tot het 2e hoofdstuk van dit proefschrift. Zowel de bachelor als de master Geneeskunde sloot Willemijn cum laude af. In 2017 kon zij dankzij financiële ondersteuning van de stichting Beat Batten en het Bartiméusfonds het onderzoek full-time voortzetten. Dit proefschrift is daarvan het resultaat. Gedurende haar promotie heeft Willemijn ook in de rol van arts-assistent patiënten met de ziekte van Batten onderzocht en gevolgd op de multidisciplinaire NCL poli, onder supervisie van Peter van Hasselt. In de toekomst hoopt zij zorg en onderzoek - in het bijzonder voor de ziekte van Batten - te kunnen blijven combineren om hiermee kinderdementie uiteindelijk de wereld uit te helpen. Willemijn woont samen met Roel in Amersfoort.

${ }^{1}$ hoewel zij inmiddels (ruimschoots) in lengte is ingehaald, blijven zij in leeftijd haar kleine broertjes. 


\section{CURRICULUM VITAE (ENGLISH)}

Willemijn Kuper was born on December $12^{\text {th }}, 1991$ in Nijmegen, the Netherlands. She studied medicine at Utrecht University from 2010 to 2016, which she finished with honors. During medical school, she started her research on the natural history of Batten disease under supervision of dr. Peter van Hasselt as part of an extra-curricular honors program. In 2017, with the financial support of the Beat Batten Foundation and the Bartiméus foundation, she continued her research on Batten disease on a full time basis under the supervision of dr. Peter van Hasselt, prof. dr. Mies van Genderen, and prof. dr. Edward Nieuwenhuis. This thesis is the result of all the work done since she started the honors program. During her PhD track, Willemijn has also examined and tracked patients with Batten disease as part of the multidisciplinary Batten disease patient clinic. For the future, she hopes to combine her research in Batten disease with her work as a medical doctor to ultimately cure childhood dementia. 

